Florida International University

FIU Digital Commons

$7-2-2020$

\title{
The Development of a Surface Enhanced Raman Spectroscopy Method for the Detection of Synthetic Cannabinoids in Urine
}

Thaddeus Mostowtt

tmost001@fiu.edu

Follow this and additional works at: https://digitalcommons.fiu.edu/etd

Part of the Analytical Chemistry Commons, and the Other Chemistry Commons

\section{Recommended Citation}

Mostowtt, Thaddeus, "The Development of a Surface Enhanced Raman Spectroscopy Method for the Detection of Synthetic Cannabinoids in Urine" (2020). FIU Electronic Theses and Dissertations. 4476. https://digitalcommons.fiu.edu/etd/4476

This work is brought to you for free and open access by the University Graduate School at FIU Digital Commons. It has been accepted for inclusion in FIU Electronic Theses and Dissertations by an authorized administrator of FIU Digital Commons. For more information, please contact dcc@fiu.edu. 


\section{FLORIDA INTERNATIONAL UNIVERSITY}

Miami, Florida

\section{THE DEVELOPMENT OF A SURFACE ENHANCED RAMAN SPECTROSCOPY METHOD FOR THE DETECTION OF SYNTHETIC CANNABINOIDS IN URINE}

A dissertation submitted in partial fulfillment of

the requirements for the degree of

DOCTOR OF PHILOSOPHY

in

CHEMISTRY

by

Thaddeus Mostowtt

2020 
To: Dean Michael R. Heithaus

College of Arts and Sciences

This dissertation, written by Thaddeus Mostowtt, and entitled The Development of a Surface Enhanced Raman Spectroscopy Method for the Detection of Synthetic Cannabinoids in Urine, having been approved in respect to style and intellectual content, is referred to you for judgment.

We have read this dissertation and recommend that it be approved.

$\begin{array}{r}\hline \text { Yong Cai } \\ \hline \text { Anthony DeCaprio } \\ \hline \text { Anthony McGoron } \\ \hline \text { Jaroslava Miksovska } \\ \hline \text { Bruce McCord, Major Professor }\end{array}$

Date of Defense: July 2, 2020

The dissertation of Thaddeus Mostowtt is approved.

Dean Michael R. Heithaus College of Arts and Sciences

Andrés Gil Vice President for Research and Economic Development and Dean of the University Graduate School

Florida International University, 2020 
(C) Copyright 2020 by Thaddeus Mostowtt

All rights reserved. 


\section{ACKNOWLEDGMENTS}

I would like to acknowledge Florida International University for their support for the six plus years I have been in the program. I would also like to thank my committee, Yong Cai, Jaroslava Miksovska, Anthony DeCaprio, and Anthony McGoron, for their input and knowledge over the course of this project and making the time for all the annual meetings and presentations. Bruce McCord, my major professor, thank you for all your help, shared knowledge, and opportunities that you provided for me while working in your research group. Thank you for your encouragement of my decision to pursue a job opportunity and continue my degree part time. Dr. Anthony McGoron, thank you for letting me use your laboratory's Raman spectrometer. Without the use of this instrument, the initial portion of this study would have not been possible. Dr. Yong Cai, thank you for letting me use your laboratories Zetasizer. Without the use of the zetasizer, gaining a better understanding of the nanoparticle and aggregating agent interaction would not have been possible. I would like to thank the National Institute of Justice (NIJ) for their financial support in the project.

To all my friends from the Mount, thank you for your words of encouragement during these six plus years. Even though we have not been able to see each other as often, I know that you are always here rooting me on like you were on the Mount tennis courts ten plus years ago. To all my friends at FIU, thank you for your help and support. We have all been through this long journey together.

Dr. Michael Epstein, after taking your forensic class at the Mount, I knew that this was a career that I wanted to pursue. Thank you for believing in me and giving me an opportunity to do conduct forensic research and introduce me to many influential people 
in various forensic fields. If it wasn't for you, I would have never meet Dr. Tony Cantu. Dr. Cantu, I probably never said this enough while you were alive, but I want to thank you for everything that you had taught me about chemistry, research, life, and food.

Mom, Dad, and Jastin, thank you for all your words of encouragement. You have always pushed me to go beyond my limits and for that I am grateful. You let me be my own person, always beat to my own drum, and encouraged me to go on new adventures. Marissa, thank you for always being supportive of all the decisions that I have made in my life, including going for my Ph.D. and moving down to Florida. I know of there are many sacrifices that you have made to help me make my dreams come true. You are my rock! 


\begin{abstract}
OF THE DISSERTATION
THE DEVELOPMENT OF A SURFACE ENHANCED RAMAN SPECTROSCOPY METHOD FOR THE DETECTION OF SYNTHETIC CANNABINOIDS IN URINE
\end{abstract}

by

Thaddeus Mostowtt

Florida International University, 2020

Miami, Florida

\title{
Professor Bruce McCord, Major Professor
}

The use and abuse of synthetic cannabinoids has increased significantly in recent years due to their easy access and growing popularity. Despite having more known drugs become illegal, new synthetic versions of these drugs are being made that have not yet been recognized or classified as illegal substances. Therefore, standard methods may not be able to detect these drugs.

The most common method of screening detection for drugs of abuse in biological samples is the immunoassay. However, the immunoassay method presents some disadvantages, particularly for newly synthesized compounds. More advanced methods have also been used, such as LC-MS; however, these procedures involve complex sample preparation and long run times.

A potential solution to this issue is surface enhanced Raman spectroscopy (SERS). When Raman spectroscopy is performed in the presence of metallic nanoparticles and aggregating agents, the signal can be enhanced several orders of magnitude via the creation hot-spots that results from displacement of the stabilizing agent, which leads to a change in the surface charge of the metallic nanoparticle and the 
ionic strength of the solution. In the present thesis an optimal SERS method was developed for the detection of synthetic cannabinoids. A variety of aggregating agents and nanoparticles were evaluated using UV/Vis spectrometry, particle sizing and zeta potential for optimal detection of these compounds. Overall, the best analytical procedure used $0.0015 \mathrm{MgCl}_{2}$ to aggregate spherical gold nanoparticles to detect synthetic cannabinoid parent compounds using a portable Raman spectrometer with a limit of detection as low as $20 \mathrm{ng} / \mathrm{mL}$ in pure samples. Then the optimized SERS method was tested on two JWH-018 metabolites producing a limit of detection as low as $37 \mathrm{ng} / \mathrm{mL}$.

To determine if the SERS method could be used for practical applications, an additional study was conducted on spiked urine samples using supported liquid extraction (SLE) prior to analysis. Since synthetic cannabinoid parent compounds are not commonly found in urine samples, two JWH-018 metabolites were examined. A few pretreatment methods prior to extraction were examined to determine the optimal SLE method to detect synthetic cannabinoid metabolites. However, the SERS spectrum of the JWH-018 metabolites after the extraction did not match that of the pure drug. Therefore, additional study needs to be conducted to determine other possible extraction methods to detect JWH-018 metabolites using SERS. 


\section{TABLE OF CONTENTS}

CHAPTER

PAGE

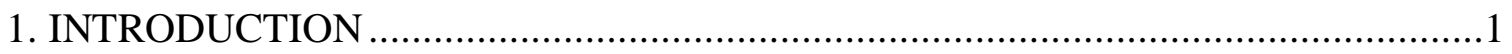

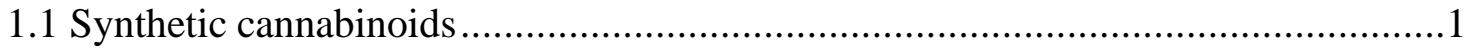

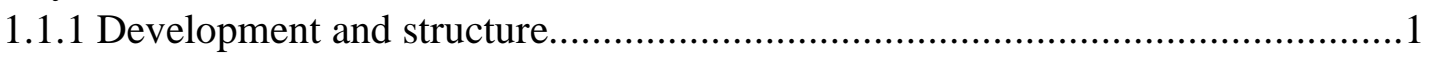

1.1.2 Pharmacology and pharmacokinetics ........................................................

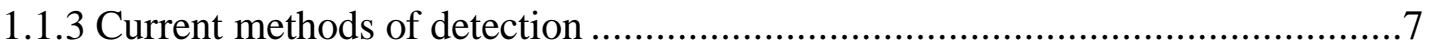

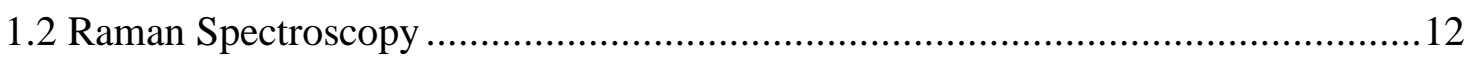

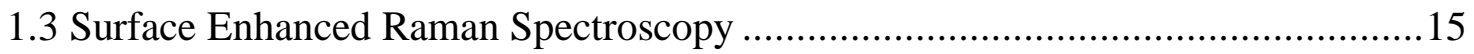

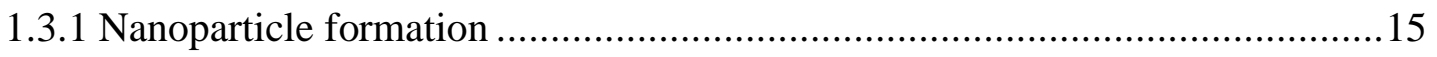

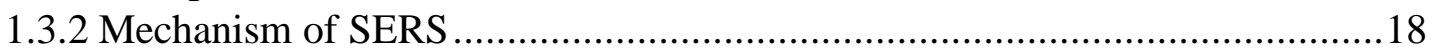

1.3.3 Improvement of enhancement through aggregation ......................................24

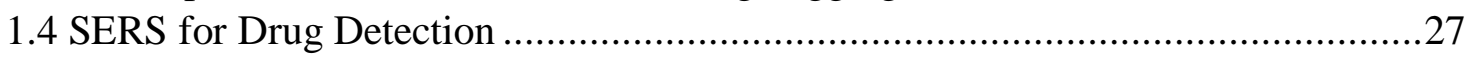

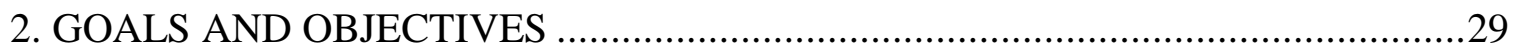

\section{INITIAL ASSESSMENT OF A SERS METHOD FOR THE DETECTION OF}

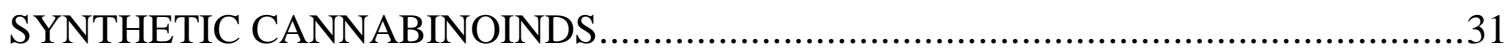

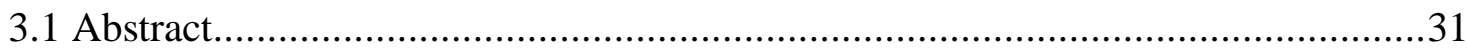

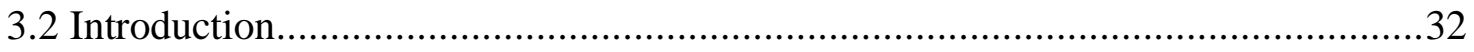

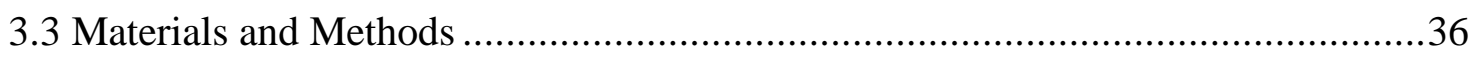

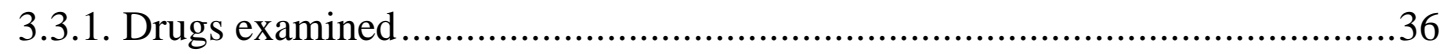

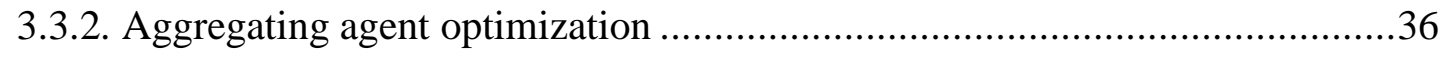

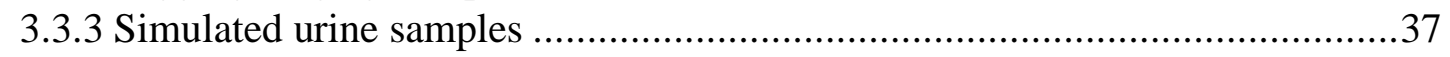

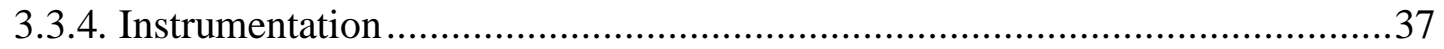

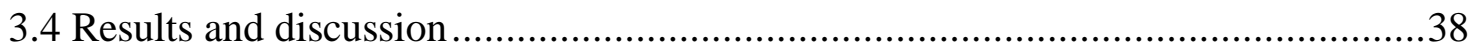

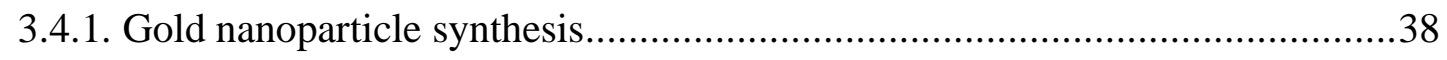

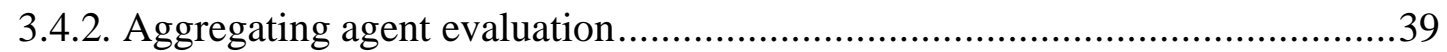

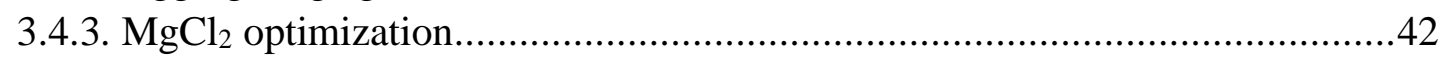

3.4.4. Bench top Raman vs. portable Raman comparison .......................................43

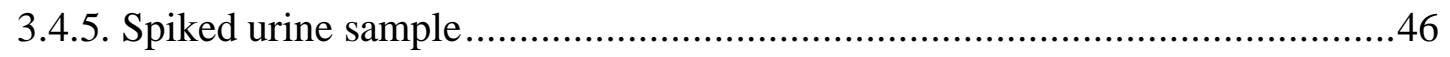

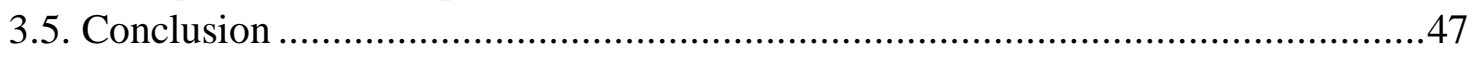

4. AN EVALUATION OF MONOVALENT, DIVALENT, AND TRIVALENT CATIONS FOR THE SERS ANALYSIS OF SYNTHETIC CANNABINOIDS .............49

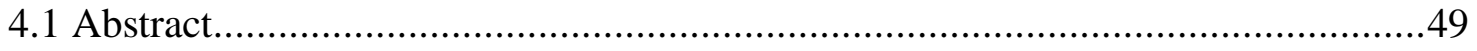

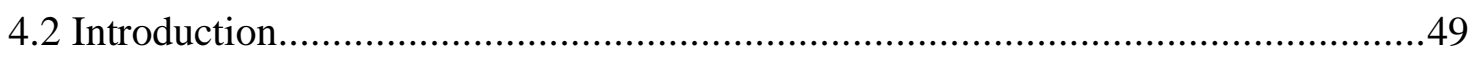

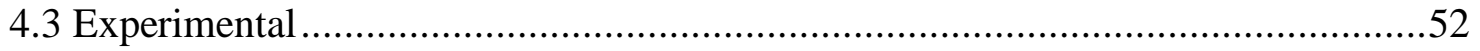

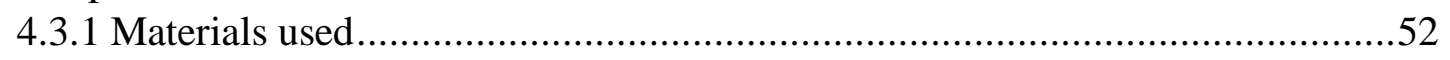

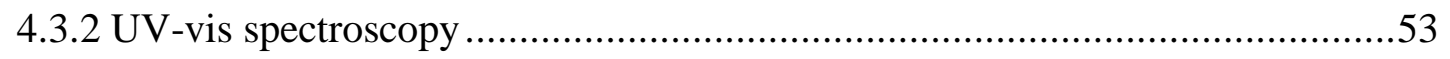




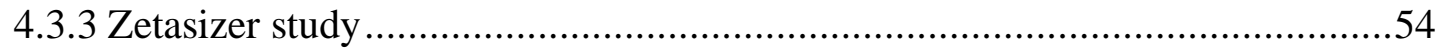

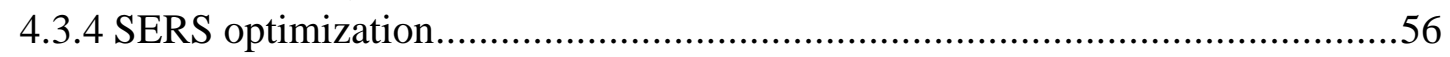

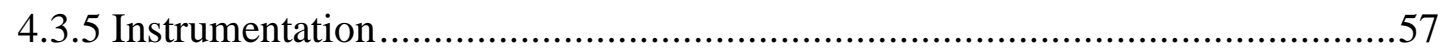

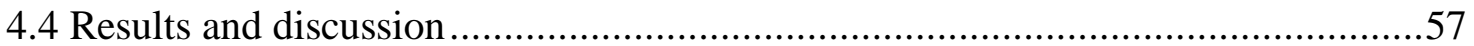

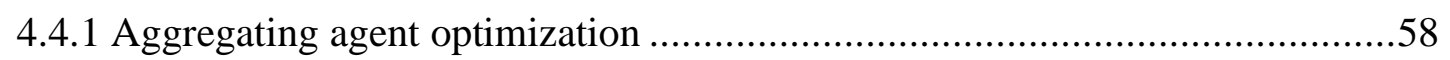

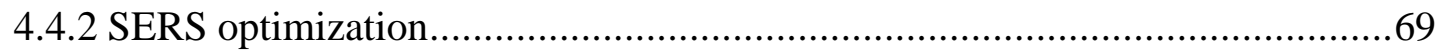

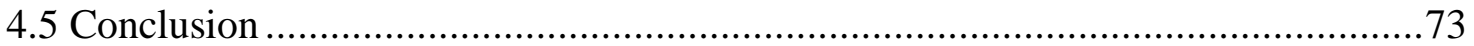

\section{THE INITIAL ASSESSMENT OF SERS FOR THE TOXICOLOGICAL} ANALYSIS OF SYNTHETIC CANNABINOID METABOLITES IN SPIKED

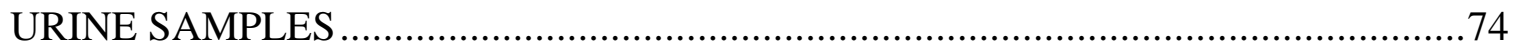

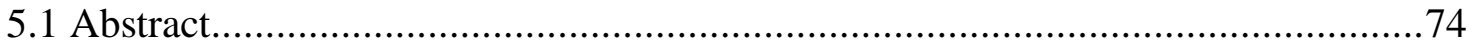

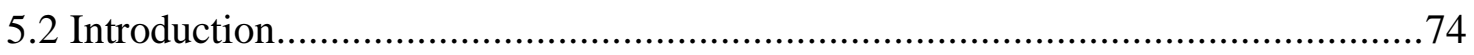

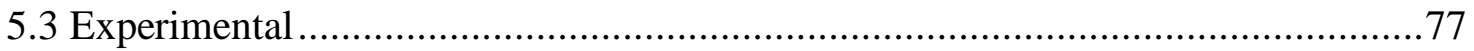

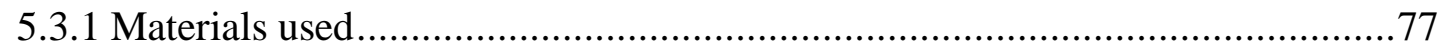

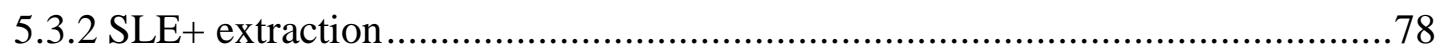

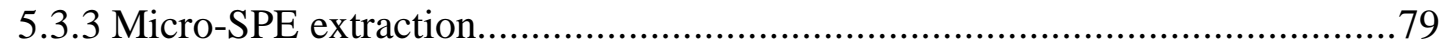

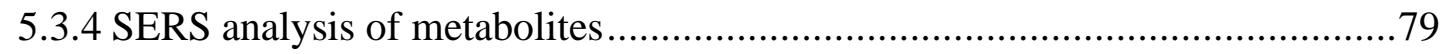

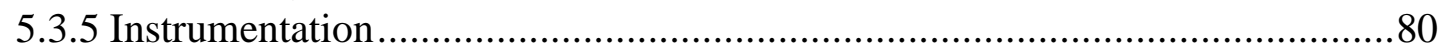

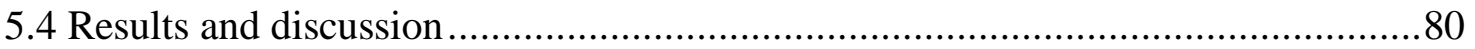

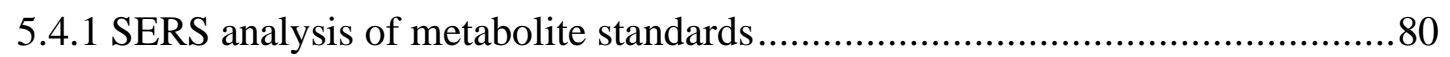

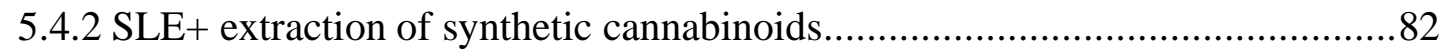

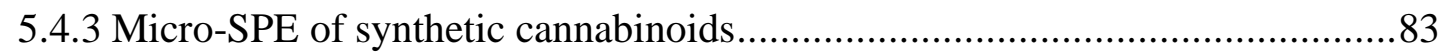

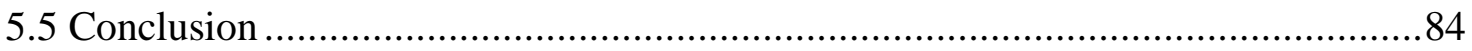

6.VIBRATIONAL SPECTRAL ANALYSIS OF SYNTHETIC CANNABINOIDS ......85

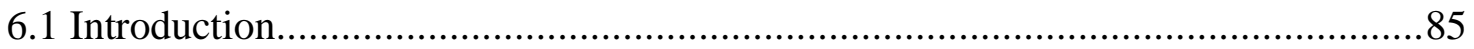

6.2 Analysis of Peaks for All synthetic cannabinoids and Major Subgroups ...............86

6.3 Spectral Analysis of additional synthetic cannabinoids examined .........................89

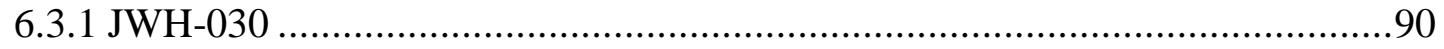

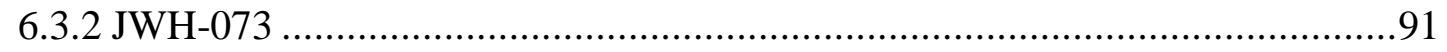

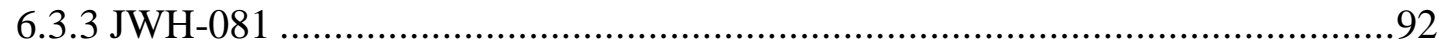

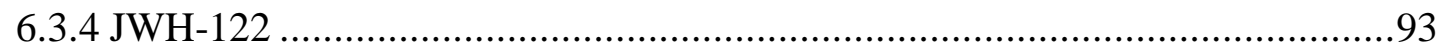

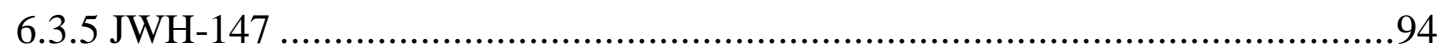

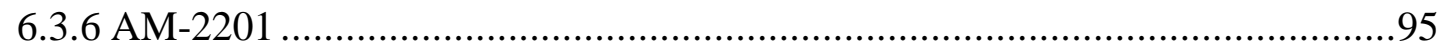

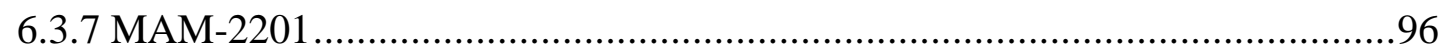

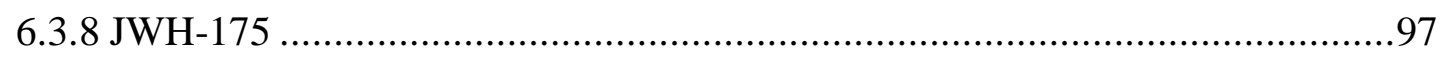

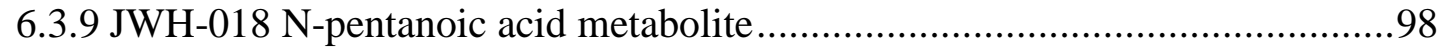

6.3.10 JWH-018 N-(5-hydroxypentyl) metabolite ................................................99

6.3.11 JWH-018 N-pentanoic acid metabolite pure drug and extraction

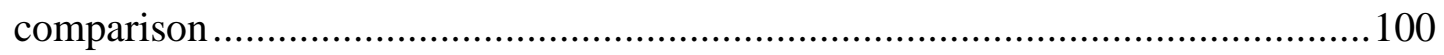


6.3.12 JWH-018 N-(5-hydroxypentyl) metabolite pure drug and extraction comparison

7. CONCLUSIONS AND FUTURE WORK …................................................. 100

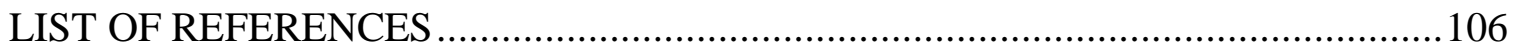

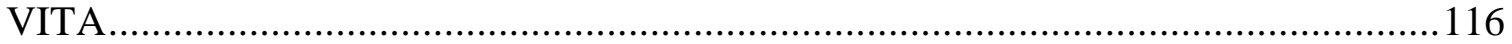




\section{LIST OF TABLES}

\section{TABLES}

PAGE

Table 1: A comparison of the effect of $0.0167 \mathrm{M} \mathrm{MgCl}_{2}, \mathrm{CaCl}_{2}$, and $\mathrm{MgSO}_{4}$ when used as aggregating agents for the detection of JWH-018, JWH-073, JWH-081, and JWH122.

Table 2: A comparison of $0.00835 \mathrm{M}, 0.0167 \mathrm{M}$, and $0.0334 \mathrm{M} \mathrm{MgCl}_{2}$ used to determine the optimal aggregating agent concentration for the detection of JWH-018, JWH-073, JWH-081, and JWH-122.

Table 3: A comparison of $0.00835 \mathrm{M}, 0.0125 \mathrm{M}, 0.0167 \mathrm{M}, 0.025 \mathrm{M}$ and $0.0334 \mathrm{M}$

$\mathrm{MgCl}_{2}$ used to determine the optimal aggregating agent concentration for the detection of JWH-018, JWH-073, JWH-081, and JWH-122 using the portable Raman spectrometer.

Table 4: (A) Initial concentration of aggregating agents examined, (B) Aggregating agent concentration at which initial color change occurred from red to purple, and (C) Aggregating agent concentration at which initial color change occurred from purple to grey/clear color.

Table 5: Characterization of the change in the average size (diameter) of the gold nanoparticles as the salt concentration increases.

Table 6: A comparison of the changes in the gold nanoparticle solution zeta potential after the addition of chloride, sulfate and nitrate salts with and without the addition of $10 \mu \mathrm{L}$ of $10 \%$ methanol $(0.02 \mathrm{M})$ solution.

Table 7: The aggregating agent used along with the calculated slope, standard deviation, $\mathrm{R}^{2}$, and limits of detection to determine the optimal aggregating agent to use with the synthesized gold nanoparticles to detect 9 different synthetic cannabinoids in solution.

Table 8: Vibrational assignment of peaks in the SERS spectrum of JWH-018.

Table 9: Vibrational assignment of peaks in the SERS spectrum of JWH-030..............90

Table 10: Vibrational assignment of peaks in the SERS spectrum of JWH-073............91

Table 11: Vibrational assignment of peaks in the SERS spectrum of JWH-081............92

Table 12: Vibrational assignment of peaks in the SERS spectrum of JWH-122............93

Table 13: Vibrational assignment of peaks in the SERS spectrum of JWH-147. 
Table 14: Vibrational assignment of peaks in the SERS spectrum of AM-2201............95

Table 15: Vibrational assignment of peaks in the SERS spectrum of MAM-2201........96

Table 16: Vibrational assignment of peaks in the SERS spectrum of JWH-175 ............97

Table 17: Vibrational assignment of peaks in the SERS spectrum of JWH-018 N-

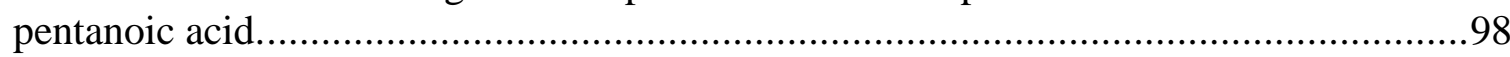

Table 18: Vibrational assignment of peaks in the SERS spectrum of JWH-018 N-(5hydroxypentyl) metabolite. 


\section{LIST OF FIGURES}

FIGURE

PAGE

Figure 1: Chemical structures of various synthetic cannabinoids. 3

Figure 2: Examples of possible metabolic pathways for JWH-073 and PB-22 (PI$\mathrm{COOH}$ is 1-pentyl-1H-indole-3-carboxylic acid) in urine. ${ }^{1}$ 6

Figure 3: A simplified Jablonski diagram showing Rayleigh, anti-Stokes, and Stokes scattering, where $\mathrm{E}_{\mathrm{L}}$ is the incident photon energy from the laser, $\mathrm{E}_{\mathrm{s}}$ is the scattered energy produced and $h \omega_{v}$ is the energy of the vibration (above). An example Raman spectrum showing the difference in signal intensity between Stokes and anti-Stokes scattering (below). ${ }^{24,25}$

Figure 4: A simplified diagram of the La Mer mechanism showing nanoparticle nucleation and growth of gold nanoparticles in solution using trisodium citrate as the reducing agent. ${ }^{29}$

Figure 5: The interaction between two gold nanoparticles producing 'hot spots' after an external applied electric field (E).

Figure 6: A basic diagram of one possible mechanism of nanoparticle destabilization via chloride ion deplacement. ${ }^{25}$

Figure 7: The chemical structure of the 4 different synthetic cannabinoids examined in this study.

Figure 8: Characterization of synthesized nanoparticles (a) UV-VIS absorption spectra;

(b) size distribution; (c) zeta potential distribution

Figure 9: Raman spectra of JWH-073 via the addition of $\mathrm{MgCl}_{2}$ (top), $\mathrm{CaCl}_{2}$ (middle) and $\mathrm{MgSO}_{4}$ (bottom) at $250 \mathrm{ng} / \mathrm{mL}$

Figure 10: The Raman spectrum produced from JWH-018, JWH-073, JWH-081, and JWH-122 using $0.0167 \mathrm{M} \mathrm{MgCl}_{2}$. The indicated peak was used to determine the LOD of each compound.

Figure 11: A comparison of the benchtop (top), portable (middle), and library (bottom) Raman spectra of JWH-018 (100 ng/mL), JWH-073 (250 ng/mL), JWH-081 (100 $\mathrm{ng} / \mathrm{mL})$, and JWH-122 (100 ng/mL) with $0.0167 \mathrm{M} \mathrm{MgCl}_{2}$ as an aggregating agent. ......46

Figure 12: The Raman spectrum of a JWH-018 standard (1000 ng/mL) using from the optimized SERS method (1), a spiked urine sample $(500 \mathrm{ng} / \mathrm{mL})$ with 1:1 water dilution (2), spiked urine sample $(500 \mathrm{ng} / \mathrm{mL})$ with 1:1 ammonium acetate buffer 
dilution (3), spiked urine sample (2000 ng/mL) without any extraction (yellow), along with background sample (4) and pure urine (5).

Figure 13: The chemical structures of the nine different synthetic cannabinoids examined in this study.

Figure 14: Characterization of the changes in absorption of the gold nanoparticle solution as concentration of $\mathrm{NaNO}_{3}$ increases.

Figure 15: Comparing changes in absorption of the gold nanoparticle solution as concentration of various chloride salts increase.

Figure 16: Characterization of the changes in absorption of the nanoparticles as concentration of the cation increases.

Figure 17: Characterization of the change in size (diameter) of the gold nanoparticles as concentration of $\mathrm{NaNO}_{3}$ increases.

Figure 18: A comparison of the SERS spectrum of ten different aggregating agents (negative controls). The negative controls contained gold nanoparticles, various aggregating agents at the concentrations noted in Table 1 Row $\mathrm{C}$, and $0.02 \mathrm{M}$ methanol.

Figure 19: A comparison of the SERS spectrum of (A) JWH-122 at $100 \mathrm{ng} \mathrm{mL}^{-1}$ with various chloride salts to that of the library spectrum of JWH-122 from the portable Raman used. The solutions examined contained gold nanoparticles, chloride salts at the concentrations noted in Table 1 Row $\mathrm{C}$ and JWH-122 in $0.02 \mathrm{M}$ methanol solution. (B) JWH-030 at $100 \mathrm{ng} \mathrm{mL}^{-1}$ with various chloride, sulfate and nitrate salts. The solutions examined contained gold nanoparticles, various chloride, sulfate and nitrate salts at the concentrations noted in Table 1 Row C, and JWH-030 in $0.02 \mathrm{M}$ methanol solution.

Figure 20: The SERS spectrum of all synthetic cannabinoids examined at $100 \mathrm{ng} \mathrm{mL}^{-1}$ in $0.02 \mathrm{M}$ methanol concentration using $0.0015 \mathrm{M} \mathrm{MgCl}_{2}$ as the aggregating agent.......72

Figure 21: The chemical structures of JWH-018 N-pentanoic acid metabolite (left) and JWH-018 N-(5-hydroxypentyl) metabolite (right) examined in this study.

Figure 22: The SERS spectrum of JWH-018 N-(5-hydroxypentyl) metabolite ranging from 2.5-2000 ng/mL (top) and The SERS spectrum of JWH-018 N-pentanoic acid metabolite ranging from $2.5-2000 \mathrm{ng} / \mathrm{mL}$ (bottom).

Figure 23: A plot of Raman intensity of a specific peak for each JWH-018 metabolite (pure drug) versus the concentration. 
Figure 24: Comparison of the SERS spectrum of JWH-018 (100 ng/mL), JWH-018 (N-5-hydroxypentyl) metabolite $(100 \mathrm{ng} / \mathrm{mL})$, and JWH-018 N-pentanoic acid metabolite $(500 \mathrm{ng} / \mathrm{mL})$ in $10 \%$ methanol.

Figure 25: Comparison of the SERS spectrum of JWH-018 N-pentanoic acid metabolite $(500 \mathrm{ng} / \mathrm{mL})$ prior to extraction and JWH-018 N-pentanoic acid metabolite spiked urine sample $(1000 \mathrm{ng} / \mathrm{mL})$ pretreated with $1 \mathrm{~mL}$ of $50 \mathrm{mM} \mathrm{NH} 4 \mathrm{OAc}$ buffer solution (adjusted to $\mathrm{pH} 4$ with $1 \%$ formic acid).

Figure 26: A comparison of the SERS spectrum of the JWH-018 N-(5-hydroxypentyl) metabolite as the pure drug $(100 \mathrm{ng} / \mathrm{mL})$ and after $\mu$-SPE extraction $(375 \mathrm{ng} / \mathrm{mL})$ as well as JWH-018 N-pentanoic acid metabolite as the pure drug $(250 \mathrm{ng} / \mathrm{mL})$ and after $\mu-\mathrm{SPE}(375 \mathrm{ng} / \mathrm{mL})$.

Figure 27: The identification of the four major groups of aminoalkylindoles (JWH073): linked group (red), linker (green), core group (blue), and tail (purple). ${ }^{87}$ .86

Figure 28: Chemical structure and SERS spectrum of JWH-018 .88

Figure 29: Chemical structure and SERS spectrum of JWH-030. .90

Figure 30: Chemical structure and SERS spectrum of JWH-073. .91

Figure 31: Chemical structure and SERS spectrum of JWH-081............................92

Figure 32: Chemical structure and SERS spectrum of JWH-122 ...........................93

Figure 33: Chemical structure and SERS spectrum of JWH-147 ..............................94

Figure 34:Chemical structure and SERS spectrum of AM-2201_............................95

Figure 35: Chemical structure and SERS spectrum of MAM-2201..........................96

Figure 36: Chemical structure and SERS spectrum of JWH-175. ...........................97

Figure 37: Chemical structure and SERS spectrum of JWH-018 N-pentanoic acid........98

Figure 38: Chemical structure and SERS spectrum of JWH-018 N-(5-hydroxypentyl) metabolite.

Figure 39:A comparison of the SERS spectrum of JWH-018 N-pentanoic acid metabolite as the pure drug and in a spiked urine sample after $\mu$-SPE.

Figure 40: A comparison of the SERS spectrum of JWH-018 N-(5-hydroxypentyl) metabolite as the pure drug and in a spiked urine sample after $\mu$-SPE. 


\section{ABBREVIATIONS AND ACRONYMS}

$\mathrm{AgNO}_{3} \quad$ Silver nitrate

Al Aluminum

$\mathrm{AlCl}_{3} \quad$ Aluminum chloride

$\mathrm{Al}\left(\mathrm{NO}_{3}\right)_{3} \quad$ Aluminum nitrate

$\mathrm{Al}_{2}\left(\mathrm{SO}_{4}\right)_{3} \quad$ Aluminum sulfate

AM Alexandros Makriyannis

$\mathrm{Au} \quad$ Gold

C Celsius

$\mathrm{Ca} \quad$ Calcium

$\mathrm{CaCl}_{2} \quad$ Calcium chloride

$\left.\mathrm{CaNO}_{3}\right)_{2} \quad$ Calcium nitrate

$\mathrm{CaSO}_{4} \quad$ Calcium sulfate

$\mathrm{CB}_{1} \quad$ Cannabinoid receptor 1

$\mathrm{CB}_{2} \quad$ Cannabinoid receptor 2

CCC critical coagulation concentration

CCIS critical coagulation ionic strength

CCD Charge coupled device

$\mathrm{Cl} \quad$ Chloride

$\mathrm{Cm} \quad$ centimeter

$\mathrm{CP} \quad$ Pfizer

DCM Dichloromethane

Dev Deviation 


\begin{tabular}{ll} 
DFSA & Drug facilitated sexual assaults \\
DLS & Dynamic light scattering \\
DLVO & Derjaguin, Landau, Verwey, and Overbeek \\
dm & decimeter \\
DNA & Deoxyribonucleic acid \\
ELISA & Enzyme-linked immunosorbent assay \\
EMIT & Enzyme-multiplied immunoassay technique \\
FWHM & Full width at half maximum \\
GABA & Gama-amino butyric acid \\
GC & Gas chromatography \\
GHB & Gamma-hydroxybutyric acid \\
GBL & Gamma-butyrolactone \\
G6P-DH & 6-phosphate dehydrogenase \\
HAuCl & Tetrachloroauric acid \\
HCl & Hydrochloric acid \\
HEIA & Homogeneous enzyme immunoassay \\
HPAg & Hydroxylamine phosphate silver \\
HPLC & High performance liquid chromatography \\
HU & Hebrew University \\
IR & Infrared \\
JWH & John W. Huffman \\
K & Potassium \\
\hline
\end{tabular}




\begin{tabular}{|c|c|}
\hline $\mathrm{KNO}_{3}$ & Potassium nitrate \\
\hline $\mathrm{K}_{2} \mathrm{SO}_{4}$ & Potassium sulfate \\
\hline $\mathrm{L}$ & Liter \\
\hline LC & Liquid chromatography \\
\hline LFIEF & Local field intensity enhancement factor \\
\hline LLE & Liquid-liquid extraction \\
\hline LOD & Limit of detection \\
\hline LSP & Localized surface plasmon \\
\hline $\mathrm{m}$ & moderate \\
\hline $\mathrm{mM}$ & millimolar \\
\hline M & Molarity \\
\hline MDMA & 3,4-methylenedioxymethamphetamine \\
\hline$\mu \mathrm{g}$ & Microgram \\
\hline$\mu \mathrm{L}$ & Microliter \\
\hline $\mathrm{mg}$ & Milligram \\
\hline $\mathrm{Mg}$ & Magnesium \\
\hline $\mathrm{MgCl}_{2}$ & Magnesium chloride \\
\hline $\mathrm{Mg}\left(\mathrm{NO}_{3}\right)_{2}$ & Magnesium nitrate \\
\hline $\mathrm{MgSO}_{4}$ & Magnesium sulfate \\
\hline $\mathrm{mL}$ & Milliliter \\
\hline mol & mole \\
\hline MS & Mass spectrometry \\
\hline MS/MS & Tandem mass spectrometry \\
\hline
\end{tabular}




\begin{tabular}{|c|c|}
\hline $\mathrm{mV}$ & Millivolts \\
\hline $\mathrm{mW}$ & Milliwatts \\
\hline $\mathrm{Na}$ & Sodium \\
\hline $\mathrm{NaCl}$ & Sodium chloride \\
\hline NAD & Nicotinamide adenine dinucleotide \\
\hline $\mathrm{NaNO}_{3}$ & Sodium nitrate \\
\hline $\mathrm{Na}_{2} \mathrm{SO}_{4}$ & Sodium sulfate \\
\hline ng & Nanogram \\
\hline NIDA & National Institute on Drug Abuse \\
\hline NH4OAc & Ammonium acetate \\
\hline $\mathrm{nm}$ & Nanomters \\
\hline NPS & Novel psychoactive substances \\
\hline PCR & Polymerase Chain Reaction \\
\hline RDX & 1,3,5-trinitro-1,3,5-triazine \\
\hline RNA & Ribonucleic acid \\
\hline $\mathrm{s}$ & strong or second \\
\hline SALLE & Salting-out liquid-liquid extraction \\
\hline SERS & Surface enhanced Raman spectroscopy \\
\hline SLE & Supported liquid extraction \\
\hline SPE & Solid phase extraction \\
\hline str & stretch \\
\hline $\mathrm{THC}$ & Tetrahydrocannabinol \\
\hline UV-vis & Ultra-violet visible spectroscopy \\
\hline
\end{tabular}


V

vibr

VW

W
Voltage

vibrations

very weak

weak 


\section{INTRODUCTION}

Synthetic cannabinoids are novel psychoactive drugs which were developed to mimic the effects of marijuana. Control and detection of these drugs is an ongoing societal problem. Many of these drugs have been adapted, developed and their structures modified in clandestine labs. These laboratories respond to government controls by developing new synthetic analogs. As current synthetic cannabinoids become controlled, newer compounds are being developed, with unknown physiological effects and potency. Currently utilized detection methods such as immunoassays and targeted HPLC/mass spectrometry can miss new developed analogs as the chemical structures keep changing. Therefore, improved methods are needed that can detect these drugs quickly with a universal detection method. The spectral information obtained via Raman spectroscopy could be useful in the presumptive identification of unknown compounds, particularly if combined with a more sensitive detection method such as surface enhanced Raman spectroscopy. Thus, the goal of my dissertation was to develop a rapid screening technique for the detection of synthetic cannabinoids at low physiological concentrations via surface enhanced Raman spectroscopy (SERS).

\subsection{Synthetic cannabinoids}

\subsubsection{Development and structure}

Synthetic cannabinoids are a member of the larger and ever-growing category of designer drugs, also known as novel psychoactive substances (NPS). ${ }^{1}$ The use and abuse of synthetic cannabinoids was initially identified in the early 2000s. ${ }^{1}$ As new synthetic cannabinoids become scheduled and made illegal by law enforcement, newer cannabinoids are being synthesized and substituted. Synthetic cannabinoids, known as 
"K2" or "Spice" were initially sold as smokable compounds that were sprayed or dried on plant material. ${ }^{1-3}$

The first synthetic cannabinoids were developed in the 1960s. ${ }^{1}$ Over the past 60 years the numbers and types of these compounds has been growing and evolving. The most common classifications for synthetic cannabinoids are classical, nonclassical, hybrid, aminoalkylindoles, phenylacetylindoles, tetramethylcyclopropylindoles, adamantoylindoles, indazole carboxamides, and quinolinyl esters. ${ }^{1-5}$ Classical cannabinoids are those with a similar structure to that of delta-9-THC, such as HU-210. Nonclassical synthetic cannabinoids are compounds that are derived from cyclohexylphenol, such as CP 47,497. Hybrid compounds contain structural similarity to both classical and nonclassical synthetic cannabinoids such as AM-4030.

Adamantoylindoles are commonly split into the four subgroups of naphthoylindoles, naphthylmethylindoles, naphthoylpyroles, and naphthylmethylindenes. ${ }^{1-5}$ An example of a compound from each functional group can be seen in Figure 1. 


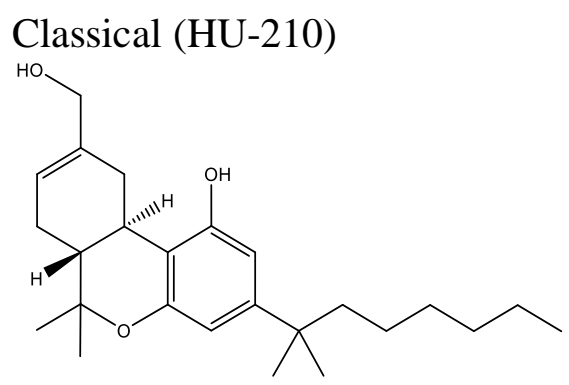

Hybrid (AM-4030)

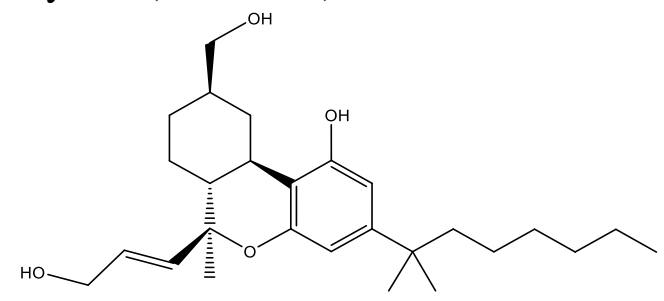

Phenylacetylindole (JWH-250)

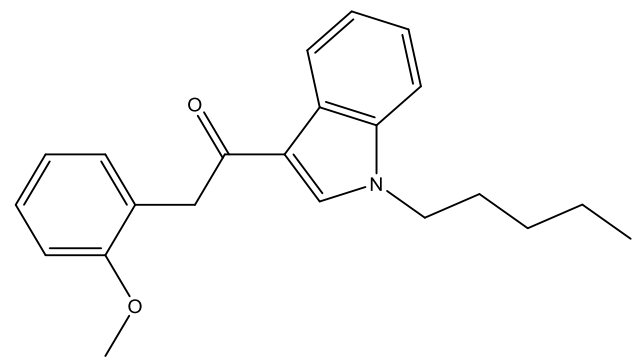

Adamantoylindole (AKB48)

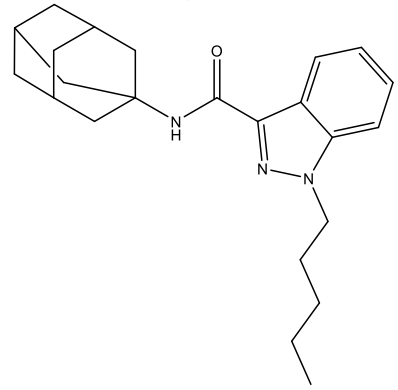

Quinolinyl ester (PB-22)

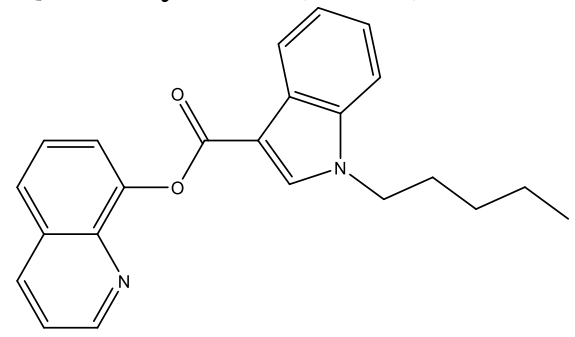

Figure 1: Chemical structures of various synthetic cannabinoids.
Nonclassical (CP 47,497)

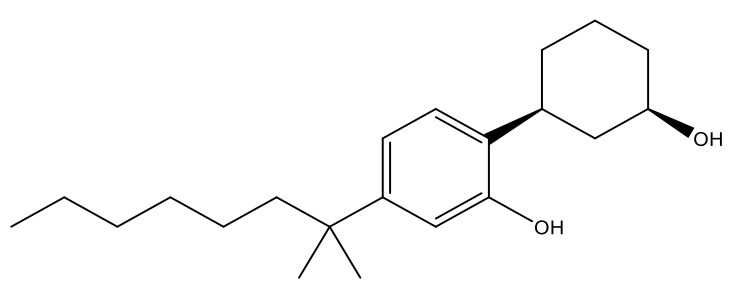

Aminoalkylindole (JWH-018)

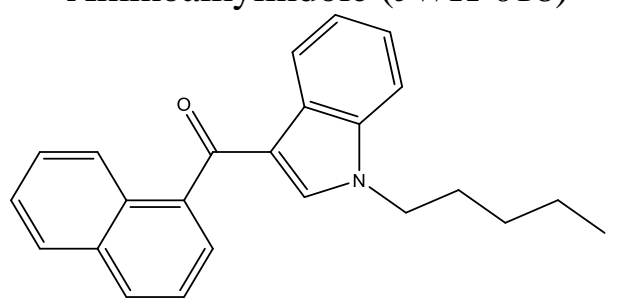

Tetramethylcyclopropylindole (XLR-11)

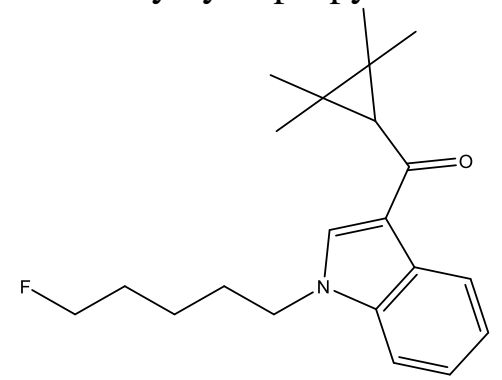

Indazole carboxamides (AB-PINACA)

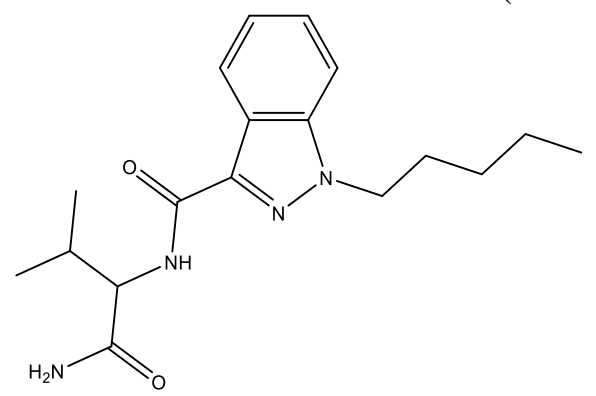


The initial naming convention of the synthetic cannabinoids was determined by the location, company, or individuals who initially developed the compounds. The HU compounds were developed at Hebrew University by Raphael Mechoulam. The CP compounds were developed by Pfizer. The AM compounds were developed by Alexandros Makriyannis. The JWH compounds were synthesized by John W. Huffman. ${ }^{3}$ Naming conventions for newer compounds, however, are determined by the chemical structure.

\subsubsection{Pharmacology and pharmacokinetics}

Synthetic cannabinoids were initially developed to identify possible medicinal uses. John W. Huffman was a part of a National Institute on Drug Abuse (NIDA) research study that investigated potential therapeutic effects. ${ }^{3}$ Synthetic cannabinoids affect the G-protein coupled receptors in the brain, which created an increase in calcium ions released into neurotransmitter ion channels with a concomitant release of potassium ions, producing neuronal hyperpolarization. ${ }^{3}$

Synthetic cannabinoids are $\mathrm{CB}_{1}$ and $\mathrm{CB}_{2}$ receptor agonists. The $\mathrm{CB}_{1}$ receptors are mainly found in the $\mathrm{G}$ protein receptors of the central nervous system. The $\mathrm{CB}_{1}$ receptors are involved in brain development, memory, pain processing, and movement control. ${ }^{2,3}$ In addition, the $\mathrm{CB}_{1}$ receptors effect glutamate and $\mathrm{GABA}$ neurotransmission. A majority of the $\mathrm{CB}_{2}$ receptors are found in the immune system and mediate immunosuppression. ${ }^{2,3}$ The metabolites of synthetic cannabinoids have been shown to influence mainly the $\mathrm{CB}_{1}$ receptors. An in vivo and in vitro study by Brents et al. showed that monohydroxylated metabolites of JWH-018 have a similar affinity to $\mathrm{CB}_{1}$ receptors as that of the parent compound or delta-9-THC, a main component found in the marijuana plant. ${ }^{6}$ Synthetic 
cannabinoids have a variety of effects not present after using marijuana, including acute toxic exposure, including tachycardia, nausea, visual and auditory hallucinations, acute tubular necrosis and possible seizures or stokes. ${ }^{2}$ Typical withdrawal effects include agitation, mood swings, loss of appetite and vomiting. ${ }^{2}$ These effects are dependent upon the absorption and distribution of the synthetic cannabinoids in the body.

Research has been conducted on the effects of absorption and distribution of synthetic cannabinoids in humans after smoking. In a study by Kacinko et al., one subject smoked an herbal incense blend containing JWH-018 and JWH-073. Blood samples were collected 19, 53, 107, and 199 minutes after smoking. The initial concentrations of each component in blood were $4.8 \mu \mathrm{g} / \mathrm{L}$ (JWH-018) and $4.2 \mu \mathrm{g} / \mathrm{L}$ (JWH-073). After 199 minutes of the concentration of each component dropped down to $0.2 \mu \mathrm{g} / \mathrm{L}$. Possible metabolites of JWH-073 were also detected. ${ }^{7}$ A study by De Jager et al. investigated the detection of JWH-018 and JWH-073 metabolites in urine from 1.5 to 65 hours after the individual smoked the blend. The metabolites that they were able to detect in the urine were JWH-018 N-pentanoic acid, JWH-073 butanoic acid, and JWH-018 N-5hydroxypentyl metabolite. ${ }^{8}$ A study by Kneisel et al. investigated the detection of synthetic cannabinoids in oral fluid and serum samples. Eleven different synthetic cannabinoids were detected in 26 different samples ranging from less than 0.1 to 457 $\mathrm{ng} / \mathrm{mL}$ in oral fluid and less than 0.1 to $8.3 \mathrm{ng} / \mathrm{mL}$ in serum samples. ${ }^{9}$ Knowing the parent compounds and metabolites present and amount of samples present in different biological matrices is important in developing a screening method to detect these and any future developed compounds. 


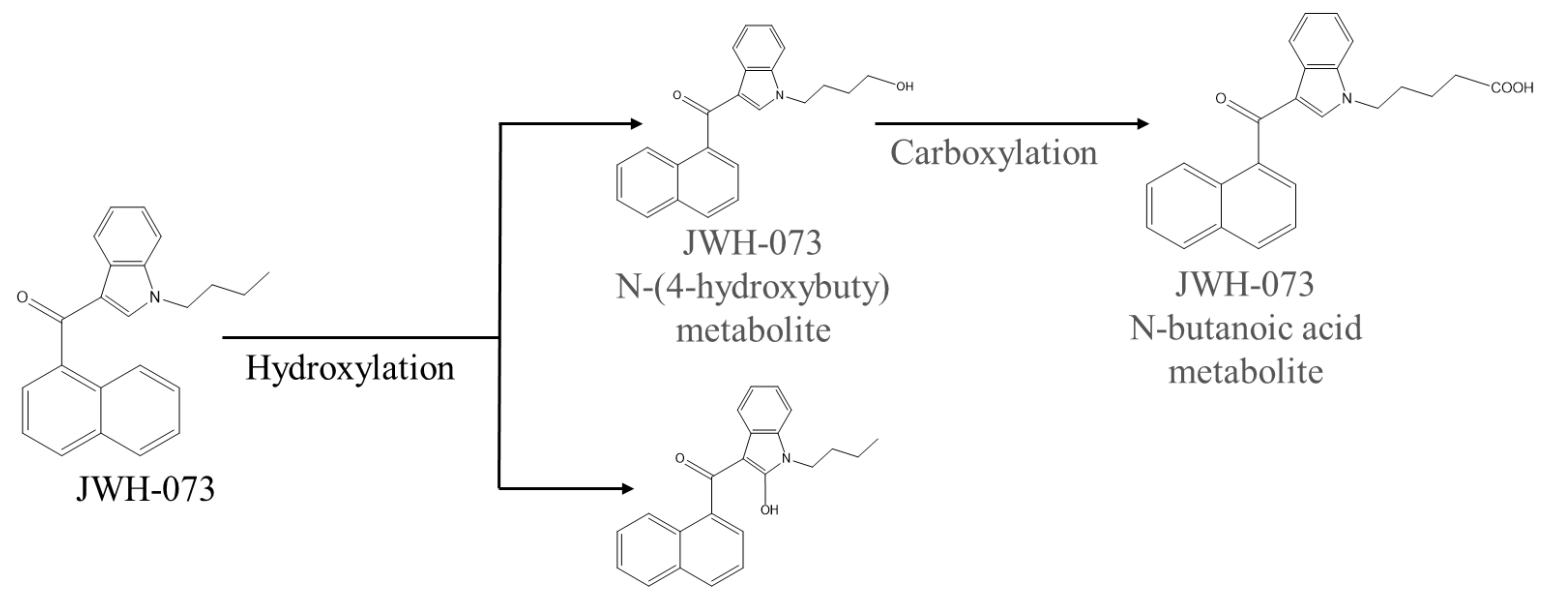

JWH-073

2-hydroxyindole

metabolite

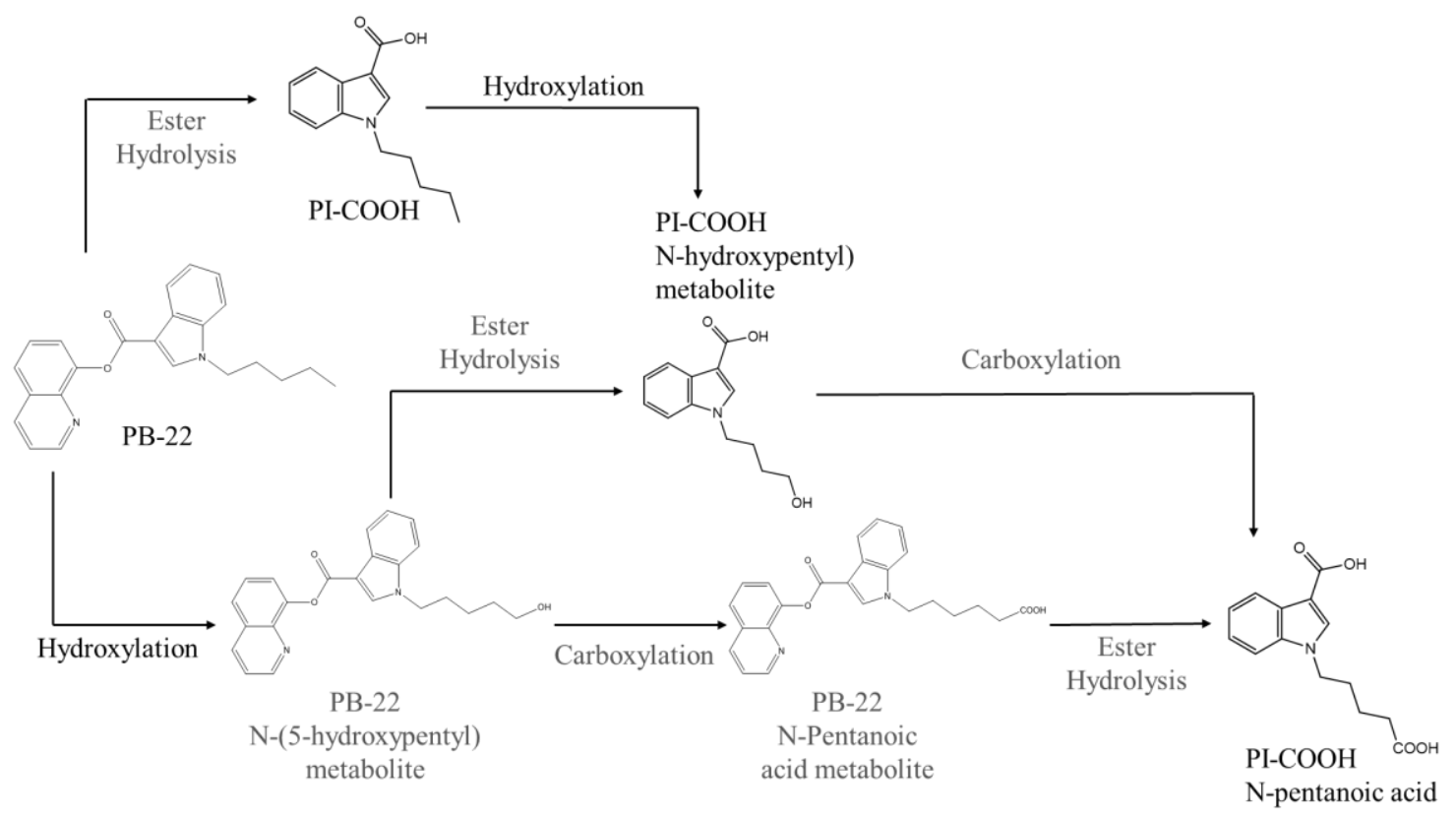

Figure 2: Examples of possible metabolic pathways for JWH-073 and PB-22 (PI-COOH is 1-pentyl-1H-indole-3-carboxylic acid) in urine. ${ }^{1}$

A variety of metabolic studies have also been conducted on synthetic cannabinoids. For the naphthoylindole compounds, the phase I metabolites occur via hydroxylation (on the idole ring or alkyl chain) or oxidative defluorination (on the alkyl chain). The phase II metabolites occur via carboxylation forming glucuronides. However, 
quinolinyl esters, such as PB-22 have a variety of metabolic pathways to include hydroxylation, carboxylation, and ester hydrolysis. 1 An example of the metabolic pathways can be seen in Figure 2 .

In the detection of synthetic cannabinoids in biological matrices, it is not easy to pinpoint unique biomarkers for these compounds. Many of these compounds share the same metabolic pathways. Some synthetic cannabinoids lose a significant part of their chemical structure undergoing biotransformation. ${ }^{1}$ To combat these issues, researchers have suggested either calculating the metabolite ratios of the major metabolites present in the sample or targeting a specific metabolite that is different between an analogue pair. ${ }^{1,10}$

\subsubsection{Current methods of detection}

Many studies have been conducted on the detection of synthetic cannabinoids in various biological matrices, such as urine, hair, blood, plasma, serum, and oral fluid. Each matrix presents its own challenges. The use of sample preparation to clean up the sample matrix prior to instrumental analysis is important because it reduces matrix interferences when detecting low level compounds. A variety of extraction techniques have been developed including hydrolysis, liquid-liquid extraction (LLE), solid phase extraction (SPE), and supported liquid extraction (SLE).

After sample extraction, screening tests are performed to determine the composition of the sample. Multiple types of tests have been analyzed for this purpose including immunoassays, gas chromatography-mass spectrometry (GC-MS), and liquid chromatography-tandem mass spectrometry (LC-MS/MS). The immunoassay is a measurement that depends on antibody and antigen interactions. ${ }^{11}$ The types of immunoassays that have been developed to detect synthetic cannabinoids include 
enzyme-linked immunosorbent assays (ELISA) and homogeneous enzyme immunoassays (HEIA). With ELISA, the capture antibody binds to the outer walls of the well plate. ${ }^{12}$ Then, the sample is added to the plate, where the analyte of interest (the antigen) binds to the capture antibody. Next, a detection antibody is added, which binds to the antigen producing a color change. The brightness of the color is proportional to the amount of the compound present in the sample. ${ }^{12}$

Rodrigues et al. determined the LOD and cross-reactivity of nineteen different synthetic cannabinoids and some of their metabolites using an ELISA immunoassay. ${ }^{13}$ The immunoassay was developed for the detection of JWH-200. Of the nineteen different synthetic cannabinoids examined, UR-144 was the only compound that could not be detected. In addition, 32 oral fluid samples were analyzed and determined to contain JWH-018, JWH-210, AM-2201, or no controlled substances/metabolites. To confirm the results of the immunoassay, the oral fluid samples were diluted with a buffer and analyzed via LC-MS/MS. The results showed that the immunoassay produced false negative results for samples with a concentration less than $0.36 \mathrm{ng} / \mathrm{mL}$. These samples were detected via LC-MS/MS. ${ }^{13}$

A study by Arntson et al. investigated the cross-reactivity of the JWH-018 Direct ELISA assay and the JWH-250 Direct ELISA assay to detect parent compounds and metabolites of various synthetic cannabinoids and other controlled substances. ${ }^{14}$ The JWH-018 Direct ELISA kit detected a total of 58 different compounds; however, the JWH-250 Direct ELISA kit detected only 16 of those same compounds. Neither assay kit detected controlled substances that were not synthetic cannabinoids. ${ }^{14}$ 
Another type of an immunoassay is homogeneous enzyme immunoassay (HEIA). Compared to ELISA, the HEIA does not require as much sample prep (no washing steps or long incubation periods), has high throughput, and exhibits low matrix effects. ${ }^{15}$ The HEIA immunoassay works similar to an enzyme-multiplied immunoassay technique (EMIT). In the HEIA immunoassay, the antibody is bound to an enzyme drug conjugate, 6-phosphate dehydrogenase (G6P-DH). The drug used is the compound that you are trying to detect. An extracted biological sample is added to the assay and competes with the drug labeled conjugate to bind to the antibody. If the sample displaces the enzyme drug conjugate, then the released conjugate is free and its attached enzyme converts nicotinamide adenine dinucleotide (NAD) to NADH, which is measured at $340 \mathrm{~nm}$. The increased displacement of the enzyme drug conjugate, yields an increase in the amount of NADH present and permits the quantitative determination of the concentration of the drug along with structurally similar compounds present in the sample. ${ }^{12,15-17}$

A study by Kronstrand et al. was conducted involving the analysis of urine samples via a Spice K2 homogeneous enzyme immunoassay (HEIA) which was capable of detecting metabolites of JWH-018, JWH-122, MAM-2201, and UR-144. ${ }^{18}$ The results showed that the authors were able to detect the various metabolites of each compound with varying degrees of cross-reactivity. However, the accuracy of the immunoassay was not acceptable at concentrations below $20 \mathrm{ng} / \mathrm{mL}$ and the precision decreased further at lower concentrations. ${ }^{18}$ Another study by Barnes et al. used the immunalysis HEIA K2 Spice kit to determine the cross-reactivity of seventy-four different compounds, including the parent drug and metabolites. Of the seventy-four tested compounds, thirty produced a low cross-reactivity (between $1-10 \%$ ) at a concentration of $500 \mu \mathrm{g} / \mathrm{L}$ and twenty-seven 
compounds produced a cross-reactivity less than $1 \% .{ }^{15}$ These results were compared to a LC-MS/MS capable of detecting all relevant drugs. Prior to analysis samples were prepared via salting-out liquid-liquid extraction (SALLE). The results of the abovementioned immunoassays demonstrated that while the developed assay kits could detect a wide range of compounds, the detection of individual compounds was highly dependent on their structure. Therefore, improved methods need to be developed that can detect a wider range of presently available compounds as well as any new compounds under development.

After samples are screened by immunoassay, gas chromatography (GC) or liquid chromatography (LC) combined with mass spectrometry (MS) are commonly used as a confirmatory test to identify synthetic cannabinoids in biological samples. Both instrumental techniques contain two steps. First, the injected sample passes through a column (the stationary phase) via a gas or liquid (mobile phase) to separate the various components in the sample. Second, a mass spectrometer detects each separated component in the sample. ${ }^{19}$ The amount of time that the sample needs to pass through the column is determined by the partitioning between the mobile and stationary phase and depends on the type phase used. After the sample components pass through the column, they enter the ion source forming ions of various mass to charge ratios. These ions pass through the mass analyzer and go to the detector. The fragmentation pattern as well as the abundance of the mass to charge ratio of the fragments obtained for each compound will vary based upon the ionization source and type of mass analyzer. ${ }^{12}$ Both single or tandem mass analyzers have been used. ${ }^{15}$ Common tandem mass analyzers contain a quadrupole 
as the first mass analyzer, a second quadrupole as a collision cell, and a quadrupole or a time of flight as a second mass analyzer. ${ }^{12}$

Many papers have been published on the identification of various synthetic cannabinoids using these methods. Kavanagh et al. analyzed urine samples of seven hospital patients thought to be under the influence of drugs. Sixteen urine metabolites of RCS-4 were identified in the patients via GC-MS. Prior to sample analysis, the urine samples underwent hydrolysis and derivatization. ${ }^{20}$ Grigoryev et al. analyzed 20 urine samples previously determined to contain UR-144 metabolites. Samples were prepared via LLE and analyzed via LC-MS/MS and GC-MS (which required sample derivatization prior to use). The authors were able to detect the mono-hydroxylated metabolites of UR144 via LC-MS/MS and GC-MS. ${ }^{21}$ A study by Heltsley et al. investigated the analysis of urine samples of US athletes. Samples were prepared via LLE or SPE and analyzed via LC-MS/MS. Out of the over 5,900 samples, 266 were positive for JW-018 or JWH-073 metabolites. The developed LC-MS/MS method had a n LOD of $1 \mu \mathrm{g} / \mathrm{L} .{ }^{22}$ Scheidweiler et al. developed an optimized LC-MS/MS method for the detection of 47 synthetic cannabinoid metabolites in urine samples. Blank urine samples were spiked with 47 synthetic cannabinoid metabolites, hydrolyzed, and extracted via SLE prior to analysis. The LOD for the developed method ranged from $0.25-20 \mu \mathrm{g} / \mathrm{L} .^{23}$

While these studies were able to detect a variety of compounds, the methods that were used have limitations. For example, when using gas chromatography, the compounds of interest need to be volatile. If a compound is not volatile, then a derivatizing agent needs to be added to the sample prior to injection. ${ }^{21}$ In addition, GCMS and LC-MS/MS have a long analysis time. Immunoassays are a quick and easy 
presumptive test; however, current immunoassays might not be able to detect newer synthesized compounds as the chemical structures for these compounds are always changing. Furthermore, variations in cross reactivity can limit detection and reduce sensitivity. Therefore, additional work is needed to develop instrumental techniques capable of detecting the currently available cannabinoids as well as those that will be developed in the future. A screening method needs to have good sensitivity and high selectivity. One current instrumental technique that can has this capability is surface enhanced Raman spectroscopy.

\subsection{Raman Spectroscopy}

Raman spectroscopy is a vibrational spectroscopic technique. A monochromatic laser beam irradiates the sample producing scattered light that is sent to the detector, where the signal is converted to a visible spectrum. The lasers commonly used in Raman spectroscopy produce an intense beam within the visible/near IR spectrum with typical wavelengths from $500-1000 \mathrm{~nm}$. The most common detectors used are multichannel charge-coupled devices (CCD), single element low band gap semiconductors, or electron multiplication CCD detectors, which help convert the data received into an electrical signal. The Raman spectra produced are measured in the infrared region of the electromagnetic spectrum (commonly $400-4000 \mathrm{~cm}^{-1}$ ). ${ }^{24,25}$ The Raman spectrum produces an individualized "chemical fingerprint" for the sample depending on the vibrational frequency of each chemical component produced via Raman scattering. 


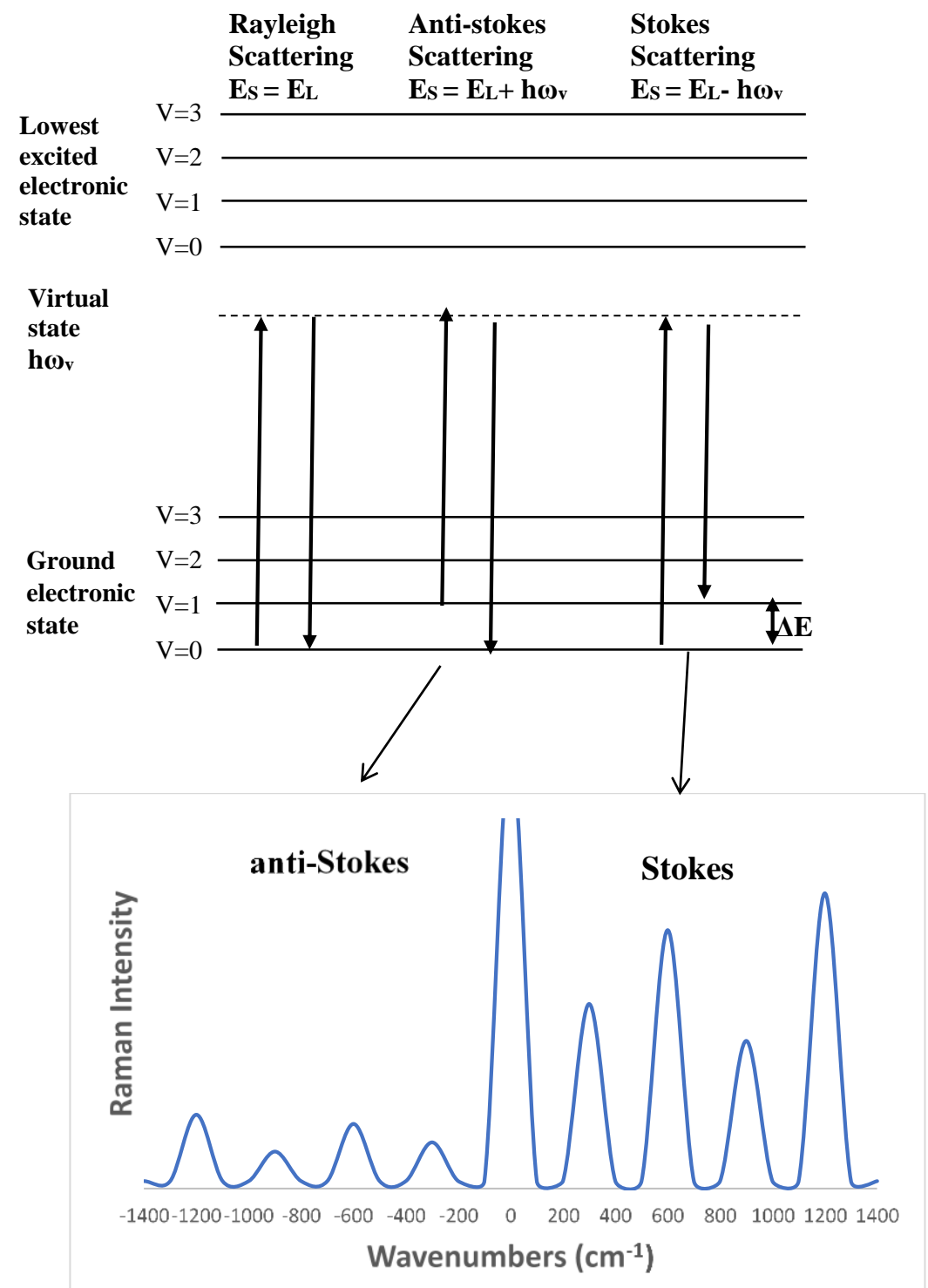

Figure 3: A simplified Jablonski diagram showing Rayleigh, anti-Stokes, and Stokes scattering, where $\mathrm{E}_{\mathrm{L}}$ is the incident photon energy from the laser, $\mathrm{E}_{\mathrm{s}}$ is the scattered energy produced and $h \omega_{v}$ is the energy of the vibration (above). An example Raman spectrum showing the difference in signal intensity between Stokes and anti-Stokes scattering (below). ${ }^{24,25}$

The scattered light produced, via the laser irradiating the sample, is elastic, (Rayleigh scattering) and inelastic (Raman scattering). In Rayleigh scattering, the molecule is excited to a virtual state and produces an emission that ends back at the original ground electronic state (Figure 3 ). ${ }^{24,25}$ Rayleigh scattering cannot be 
distinguished from the frequency of the incident beam, and therefore cannot be used for the structural interpretation of any compounds. With Raman scattering, the absorbed photon is reemitted at a different intensity that is $1 / 100000$ of the intensity of the incident beam, which results in a spectrum which is characteristic of the molecular absorbance of the molecule. ${ }^{24,25}$

The difference in the vibrational frequencies produced via Raman scattered light is measured as a series of negative (Stokes) or positive (anti-Stokes) shifts from the incident beam frequency. In Stokes scattering, the molecule is excited at the lowest energy state to a 'virtual state,' then it produces an emission that ends at the energy state higher than originally started. In anti-stokes scattering, the molecule is excited at the lowest energy state, to a similar 'virtual state,' then produces an emission that ends at the energy state lower than originally started (Figure 3 ) ${ }^{24,25}$ Since more molecules are originally located in the lower energy states, stokes scattering will produce higher intensity spectra than anti-stokes (Maxwell-Boltzmann distribution law). Therefore, the stokes side of the spectrum is typically used for analysis. ${ }^{24,25}$

The detection of a target compound via Raman spectroscopy requires a change in the polarizability of various bonds (vibrational modes) forming the compound. As the laser hits the compound, producing Raman scattering, the electrons around the various bonds in the compound are momentarily distorted, which creates a molecule that is temporarily polarized, resulting in an induced dipole. The variation in the polarizability of various vibrational modes in the compound creates results in the creation of the peaks present in the Raman spectrum. The Raman signal intensities of these peaks are directly proportional to the concentration of the molecule. ${ }^{25,26}$ The Raman spectrum produced is 
recorded in wavenumbers $\left(\mathrm{cm}^{-1}\right)$ versus signal intensity. Further detail on the spectral interpretation of the various synthetic cannabinoids examined in this study will be discussed in chapter 6.

The weakness of the conventional Raman technique is that the compound must be present at a sufficient concentration to produce a detectable Raman signal. In research conducted by Weyermann et al., samples of cocaine, heroin, and amphetamine could only be detected at concentrations as low as $1 \mathrm{mg} / \mathrm{mL}$ in diluted deionized water and drug mixtures were hard to discriminate. ${ }^{27}$ As a result, conventional Raman cannot analyze samples at physiological concentrations.

\subsection{Surface Enhanced Raman Spectroscopy}

In 1974, surface enhanced Raman spectroscopy (SERS) was developed by Fleischmann et al. which enabled the analysis of samples at physiological concentrations. In their research, an enhancement of the Raman spectrum from pyridine adsorbed on a roughened silver electrode was observed. ${ }^{28}$ Their discovery led to acceleration in the research involving Raman spectroscopy. Surface enhanced Raman spectroscopy uses a metal substrate to produce a signal enhancement that is several orders of magnitude greater than the signal produced from conventional Raman spectroscopy. The substrate may be a roughened metal surface or an aggregation of nanoparticles. There is an advantage to using nanoparticle solutions, as they provide a fresh surface with each use and signals ca be further enhanced through the use of aggregating agents.

\subsubsection{Nanoparticle formation}

Nanoparticles are formed through the coalescence and nucleation of reduced metallic salts. There are a few theories on nanoparticle nucleation and growth. The most 
common theory is the La Mer mechanism, which describes the nucleation and growth as a three-step process. ${ }^{29}$ Initially, there is a rapid formation of monomers, the free metal ion, in solution. Then, the metal ion undergoes "burst nucleation." Once an abundance of the free metal ion is formed, the metal ions close together to form nanoparticles. At the time when no additional nucleation occurs, then the existing monomers bind to the current nuclei forming larger particles. Finally, the metal ions disperse throughout the solution creating nanoparticles of various sizes (Figure 4). ${ }^{29}$ The concentration of the monomer affects the time needed for phase II to be completed. An increase in the concentration of the monomers leads to additional time needed to complete nucleation.

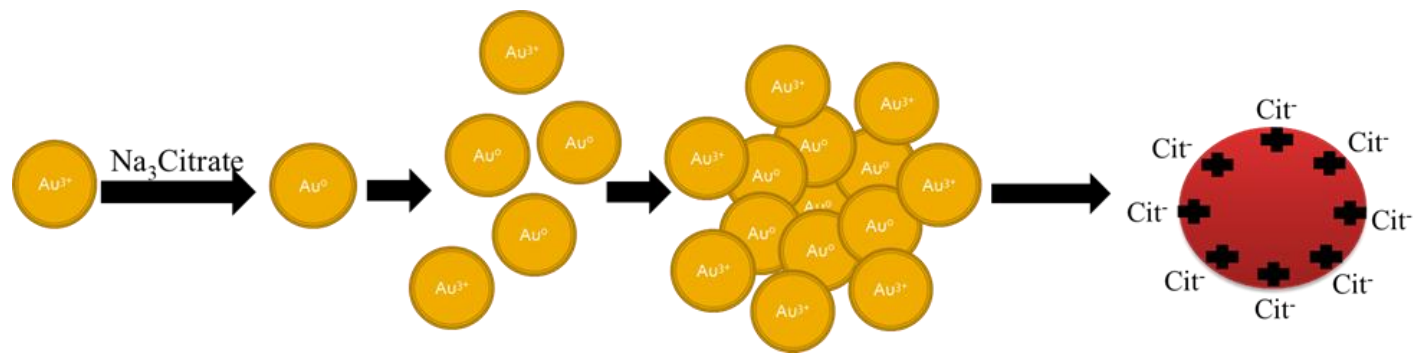

Figure 4: A simplified diagram of the La Mer mechanism showing nanoparticle nucleation and growth of gold nanoparticles in solution using trisodium citrate as the reducing agent. ${ }^{29}$

The formation of nanoparticle sizes in solution have been explained via Ostwald, as digestive, or intraparticle ripening. In Ostwald ripening, the smaller formed nanoparticles break apart in solution and bind to the bigger nanoparticles to form large nanoparticles. The above is caused by the surface energy and high solubility of the smaller nanoparticles. ${ }^{29}$ In digestive ripening, the formed nanoparticle, because of surface energy of that particle, breaks apart to form smaller nanoparticles. ${ }^{29}$ In intraparticle ripening, there is a separation of the metal ions on the outer formation of the nanoparticle, which changes the shape of the nanoparticle. The above happened because of the surface 
energy of the different monomers of the nanoparticle. ${ }^{29}$ Another mechanism to describe nanoparticle nucleation and growth is the Finke-Watzky two step mechanism. In the twostep mechanism, two events are happening simultaneously: the slow nucleation of the metal and the growth of the nanoparticle via a catalyst. ${ }^{29}$ The Finke-Watzky two step mechanism process of the nucleation and growth of gold nanoparticles cannot be conducted without the use of a reducing agent. A common reducing agent in spherical gold nanoparticle synthesis is trisodium citrate.

A study by Chow et al. showed that the concentration of the trisodium citrate added to the heated tetrachloroauric acid solution can affect the $\mathrm{pH}$ of the solution and cause two different reaction pathways for gold nanoparticle nucleation. ${ }^{30}$ By keeping the $\mathrm{pH}$ between $3.7-6.5$, the tetrachloroauric acid forms a $\left[\mathrm{AuCl}_{3}(\mathrm{OH})\right]^{-}$intermediate that undergoes a LaMer bust nucleation for less than 10 seconds, then a fast random attachment, and finishes with intraparticle ripening. If the $\mathrm{pH}$ is between 6.5-7.7, there is a reduction of the tetrachloroauric acid forming $\left[\mathrm{AuCl}_{3}(\mathrm{OH})\right]_{2}{ }^{-}$and $\left[\mathrm{AuCl}_{3}(\mathrm{OH})\right]_{3}{ }^{-}$ intermediates which undergo a long nucleation (around 60 seconds) followed by slow growth. ${ }^{30}$

Upon the completion of the nucleation and growth of the gold nanoparticles in solution, the stern (outer) layer contains the citrate trianions. Park et al. studied how the citrate anion bonds with the nanoparticles to produce an electrostatically stable nanoparticle solution using a variety of instrumental techniques. ${ }^{31}$ They concluded that a citrate layer forms from dihydrogen citrate anions adsorbing onto the gold nanoparticle surface, which hydrogen bond with other dihydrogen citrate anions already adsorbed in the gold nanoparticle surface and hydrogen bond with other citrate anions creating citrate 
chains. These formed citrate chains interact with each other via Van der Waals interactions. ${ }^{31}$ With each nanoparticle having a negatively charged stern layer, there is a steric repulsion between each nanoparticle allowing the nanoparticle solution to be electrostatically stabilized. ${ }^{31}$ To detect compounds of interest in solution, an aggregating agent is added to destabilize the nanoparticles in solution in order to produce the SERS enhancement through the development of enhanced local fields at points of interaction between the particles.

\subsubsection{Mechanism of SERS}

For the SERS effect to take place, the compound of interest must be adsorbed or within about $10 \mathrm{~nm}$ of the metallic surface to produce an electromagnetic interaction. ${ }^{25,32}$ There are two main theories on the mechanism for enhancement that occurs in SERS, which involve chemical and electromagnetic effects respectively. ${ }^{25,32}$ In both theories, the Raman signal is the result of an interaction between the induced dipole moment $(\mathrm{P})$, the electric field (E), and the polarizability $(\alpha)$ of the molecule. As the molecule interacts with the metal substrate, localized variations in the electric field alter the polarization of the molecule and its dipole moment resulting in an enhancement of the Raman signal. However, the process of enhancement can vary depending on the molecular structure and surface. The two theoretical descriptions attempt to explain these factors through electromagnetic and chemical effects.

In the electromagnetic theory, the electric field of the molecule is enhanced by the electromagnetic effect produced by the metal surface that results from the collective excitation of the electron cloud near the surface (surface plasmons). Regardless of the exact mechanism of enhancement, the excitation of the surface plasmon greatly increases 
the local field experienced by a molecule adsorbed on the surface of the metal. In the same way, the metal also enhances the Raman scattered field. ${ }^{25,32}$

First, one must understand why metals are used for detection of low concentrations of various compounds. The electrical and optical properties of a metal can be characterized by its dielectric function $\epsilon(\omega)$, where $\omega$ is the frequency of light. The frequency of light can be calculated using the equation $\omega=2 \pi c / \lambda$, where $c$ is the speed of light and $\lambda$ is the wavelength. ${ }^{32}$ Therefore, the dielectric function can be related to $\omega$ or $\lambda$. The dielectric function of a metal with respect to a specific wavelength $\epsilon(\lambda)$, at a given wavelength, is characterized by real and imaginary parts. ${ }^{32}$

Within the visible range of the electromagnetic spectrum, the real part of the dielectric function is large and negative. At long wavelengths, the real part of the dielectric function can be determined by the lossless Drude model:

$$
\epsilon=\epsilon_{\infty}\left(1-\frac{\omega_{p}^{2}}{\omega^{2}}\right)=\epsilon_{\infty}\left(1-\frac{\lambda_{p}^{2}}{\lambda^{2}}\right)
$$

where $\omega_{\mathrm{p}}$, the plasma frequency, and is equivalent to $2 \pi \mathrm{c} / \lambda_{\mathrm{p}}$. However, metals are not lossless, having no dissipation of electromagnetic energy. Therefore, there is an imaginary portion of the dielectric function that is important. ${ }^{32}$ The imaginary portion is related to the absorption of the metal. The dielectric function in relation to wavelength for gold metal contains additional contributions from free electrons, which give it a higher absorption when compared to other metals, such as silver. ${ }^{32}$ Upon understanding the dielectric function of a metal, one can understand the electromagnetic properties of the metal, such as reflectance. 
Upon an incident electromagnetic wave hitting a metal surface at a specific wave vector, reflected and transmitted waves at a specific wavevector result. The amplitude of the transmitted waves is the result of the index of refraction of the metal, which is related to the dielectric function, $n(\omega)=\sqrt{\epsilon(\omega)}$. The intensity of the reflected light is determined by the Local Field Intensity Enhancement Factor (LFIEF) ${ }^{25,32}$ The LFIEF is the normalized value of the square of the electric field amplitude at a specific point divided by the normalized intensity of the incoming field at

$$
\operatorname{LFIEF}(r)=|E(r)|^{2} /\left|E_{0}(r)\right|^{2}
$$

that specific point. The LFIEF is dependent upon the frequency and wavelength at the specific point and therefore the LFIEF can be rewritten as:

$$
\operatorname{LFIEF}(r, \omega)=|E(r, \omega)|^{2} /\left|E_{0}(r, \omega)\right|^{2}
$$

The LFIEF could be greater than or less than 1, depending upon if the local field is enhanced or quenched. ${ }^{25,32}$

In a traditional sense, the LFIEF on a flat surface for a normal incidence excitation of a bulk metal, such as gold, from air would be quenched. However, if the gold was a lossless metal, an electromagnetic field would be created on the surface of the metal. If the metal is lossless, then there is no dissipation of electromagnetic energy. When the incident wave from the laser hits the electromagnetic field of the metal, it sends the incident electromagnetic wave in the opposite direction. ${ }^{25,32}$ However, at high reflectivity, a low LFIEF can be achieved on the surface of the metal. Other LFIEFs can be achieved at different angles, which are dependent upon the incident angle of polarization. ${ }^{25,32}$ In addition, the type of metal used, and its shape can have an effect on the local electric field. 
In my study, spherical gold nanoparticles were used to produce the SERS enhancement. To gain an understanding of what happens to the electromagnetic field around the gold nanoparticle, Maxwell's equations can be used with set boundary conditions. ${ }^{25,32}$ To simplify the mathematical solutions, electrostatic approximation can be used to explain the effects of an electromagnetic field on a metal. In electrostatic approximation, the applied electric field of the metal has a uniform field that oscillates at a specific frequency, but not in a direction of a specific vector or wavelength. The electrostatic approximation is valid for nanoparticles less than $10 \mathrm{~nm}$; however, it can be applied for larger objects. ${ }^{25,32}$ The electrostatic problem of a sphere can be solved understanding that the sphere has an induced dipole at a specific origin $\rho$ and surrounding the nanoparticle is an applied external field. The magnitude of the induced dipole can be calculated as follows:

$$
\rho \propto\left(\frac{\epsilon(\lambda)-\epsilon_{M}}{\epsilon(\lambda)+2 \epsilon_{M}}\right)
$$

The dielectric function $\epsilon(\lambda)$ is a complex number having a real, $\operatorname{Re}[\epsilon(\lambda)]$, and imaginary, $\operatorname{Im}[\epsilon(\lambda)]$, portion. While the real portion of the dielectric function can achieve $\operatorname{Re}[\epsilon(\lambda)]=$ $-2 \epsilon_{\mathrm{M}}$ (implying that $\rho \rightarrow \infty$ ), this cannot be achieved by the imaginary portion of the dielectric function. Therefore, the magnitude of $\operatorname{Im}[\epsilon(\lambda)]$, however small, is the magnitude of $\rho$ at a specific wavelength and called the localized surface plasmon resonance of the sphere. ${ }^{25,32}$

The part of the spherical nanoparticle that has the largest local field enhancement are the two points, in the direction of the external electric field, that go through the center of the sphere. The LFIEF at a specific area of a spherical nanoparticle can be calculated 
and will occur over a specific wavelength range, where $\operatorname{Re}[\epsilon(\lambda)]=-2 \epsilon_{M}$ is satisfied, based upon the metal used. ${ }^{25,32}$ For example, the LFIEF's can be estimated at a specific point on gold and silver spherical nanoparticles. While the LFIEF has a higher signal intensity for silver over gold nanoparticles, gold has a broader wavelength range where $\operatorname{Re}[\epsilon(\lambda)]=-$ $2 \epsilon_{\mathrm{M}}$ can be achieved. The intensity of excitation over this broad wavelength range for gold spherical nanoparticles is smaller due to the larger $\operatorname{Im}[\epsilon(\lambda)]$ at the corresponding resonance frequency. ${ }^{25,32}$

Another factor to consider is the size of the nanoparticle. The electrostatic approximation works for nanoparticles less than $10 \mathrm{~nm}$. Nanoparticles ranging from 30 to $100 \mathrm{~nm}$ can have size effects. First, the LSP resonance varies depending upon the size of the nanoparticle. As the nanoparticle size increases, the LSP resonance shifts to a higher wavelength (red shift). Second, the LFIEF decreases as the nanoparticle size increases, broadening the resonance. Third, the nanoparticles ranging from 30 to $100 \mathrm{~nm}$ produce multipolar resonances that do not effectively couple with incident radiation to produce a strong LFIEF. $^{25,32}$

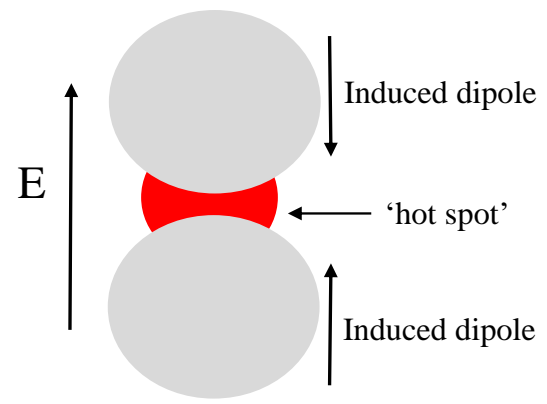

Figure 5: The interaction between two gold nanoparticles producing 'hot spots' after an external applied electric field (E). 
In addition to the effect of a single particle with plasmon resonance, the interaction between two or more close particles (coupled plasmon resonance) can affect SERS enhancement. The interaction between the two particles is caused by the induced dipoles of the two or more particles. These interactions create electromagnetic 'hot spots' (Figure 5). The closer the particles are to each other, an increase in the intensity of the local field intensity enhancement occurs, creating a stronger Raman signal intensity that produces a red shift of the Raman spectrum. ${ }^{25,32}$ The highest signal intensity is located at the area of closest contact between the two particles. A variety of metallic substrates in various forms have been tested in surface enhance Raman spectroscopy, which includes gold, copper, silver, and alkali metals. Of those metals, the most prominently used metals have been gold and silver because of their strong plasmon resonance. ${ }^{25}$

In addition to the electromagnetic theory, there are other factors, such as chemical enhancement, that affects SERS enhancement. Chemical enhancement is the change in the polarizability of the metal nanoparticle following adsorption of the compound of interest onto the metal nanoparticle surface. Chemical enhancement can result in the quenching (not being able to see the compound of interest) or enhancement (via a chargetransfer mechanism) of the SERS signal. ${ }^{25}$ There are three different ways at which charge-transfer mechanism can be explained. First, the metal nanoparticle does not bind covalently to the analyte of interest, but causes a change in the electronic distribution of the analyte, which changes the Raman polarizability. ${ }^{25}$ Second, the addition of a surface complex could covalently bind to the metal or indirectly binds to the metal via an electrolyte ion and change the polarizability. Chloride is an example of an electrolyte ion. Third, is a photon-driven charge transfer between the metal and the analyte of interest. ${ }^{25}$ 


\subsubsection{Improvement of enhancement through aggregation}

As stated previously, spherical gold nanoparticles can be formed using sodium citrate as the reducing agent, which creates stabilized negatively charged nanoparticles. In order to detect the compound of interest via SERS enhancement, the nanoparticles need to become destabilized to create electromagnetic 'hot spots.' One way to destabilize the nanoparticles is the use of an aggregating agent.

Despite having some understanding about aggregation, the exact mechanism for which destabilization is still unknown. One theory is that the anion of the aggregating agent displaces the citrate surrounding the nanoparticle, reducing the repulsion between particles. The above theory is thought occur specifically with chloride and other salts (Figure 6) ${ }^{25}$ However, as important as the anion is to nanoparticle destabilization, the cation could also have an effect.
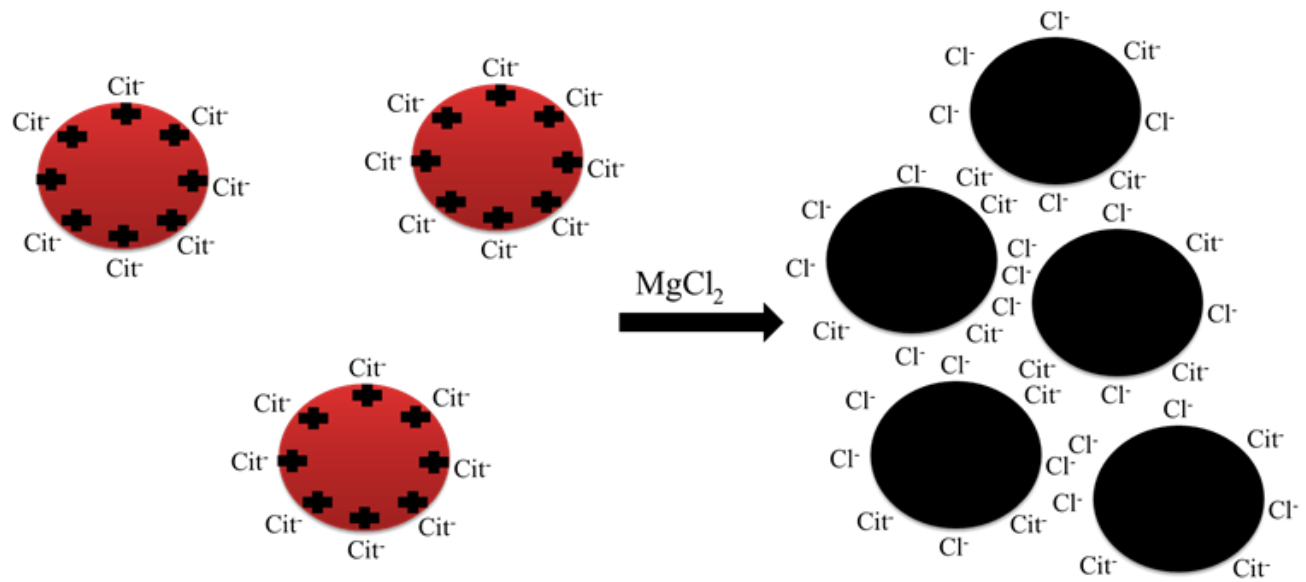

Figure 6: A basic diagram of one possible mechanism of nanoparticle destabilization via chloride ion deplacement. ${ }^{25}$

A variety of studies have been published on the destabilization of gold nanoparticles using various salts. A study by Kim et al. looked at the change in absorbance, nanoparticle size, and zeta potential with the use of benzyl mercaptan as the 
aggregating agent. The peak of the surface plasmon band of gold nanoparticles via sodium citrate reduction is typically around $520 \mathrm{~nm}$. After the addition of the aggregating agent, there was a red shift in the UV-vis spectrum, shifting the surface plasmon band to $720 \mathrm{~nm}$, and continuing to shift to higher wavelengths as the concentration of the benzyl mercaptan increased. ${ }^{33}$ As the concentration of the benzyl mercaptan increased, the average nanoparticle size increased and the zeta potential decreased. In addition, there was a color change from red to blue after the addition of the aggregating agent. The authors believed that the addition of the benzyl mercaptan to the nanoparticle solution caused the benzyl mercaptan to displace the citrate ions surrounding the nanoparticles causing a change in the stability ratio and bringing the nanoparticle closer together for SERS enhancement. ${ }^{33}$

Another factor involves the effect of an aggregating agent's ionic strength on the color change that occurs during nanoparticle destabilization. Burns et al. titrated various monovalent, divalent, and trivalent sulfate, nitrate, phosphate, and chloride salts into a gold nanoparticle solution until a color change occurred. Prior to and at the titration end point, the UV-vis absorbance, nanoparticle size, and zeta potential were recorded. ${ }^{34}$ The concentration of the aggregating agent at the titration end point was determined. From the above information, the ionic strength, activity coefficient, and Debye length (nm) were calculated. The Debye length is the calculated particle-to-particle mean distance between nanoparticles in solution. ${ }^{34}$

The results showed that the multivalent salts reached the titration endpoint at a lower concentration than monovalent salts. The same trend was seen with ionic strength. However, there was no correlation to Debye length. The particle-to-particle mean 
distance varied between monovalent, divalent, and trivalent salts and was not related to the color change of the nanoparticle solution. ${ }^{34}$

Similar to Kim et al., there was a red to blue color change of the nanoparticle solution as a result of a red shift in the UV-vis spectrum, and a decrease in the zeta potential. However, the zeta potential changed from a negative to a positive number at the titration endpoint. The charge of the zeta potential of a nanoparticle solution is related to the charge of the reducing agent. ${ }^{34}$ If gold nanoparticles were formed via a sodium citrate reduction, then the zeta potential would be is negative. The change from negative to positive means that the stern layer of the nanoparticle has become positively charged after the addition of the aggregating agent. Therefore, the authors believed that the cation of the aggregating agent influences nanoparticle aggregation and the color change was the result of the cation surface absorption. ${ }^{34}$ The results of these two studies, show that aggregation of the nanoparticles could be caused by both the cation and the anion of the aggregating agent.

Another way to examine the effect of monovalent cations and various anions of aggregating agents is to utilize the Hofmeister series as a driver for critical coagulation concentration (CCC). The Hofmeister series is a list of specific cations and anions with respect to their capability to salt-out or salt-in proteins. The CCC is the demarcation between fast and slow nanoparticle aggregation. A study by Oncsik et al. determined the CCC for monovalent salts when mixed with positively and negatively charged latex nanoparticles. The more hydrated cations and anions reached the $\mathrm{CCC}$ at a higher concentration than the least hydrated cations and anions. ${ }^{35}$ Additional work by Pavlovic et al. studied the $\mathrm{CCC}$ for multivalent anions. For $\mathrm{PO}_{4}{ }^{3-}, \mathrm{Fe}(\mathrm{CN})_{6}{ }^{3-}$, and $\mathrm{Fe}(\mathrm{CN})_{6}{ }^{4-}$, more 
than one $\mathrm{CCC}$ was discovered as the concentration of salts with those anions increased when added to $\mathrm{MgAl}-\mathrm{NO}_{3}-\mathrm{LDH}$ particles. The authors believed that it could have been a consequence of hydrogen bonding, hydrophobic interactions, or electrostatic interactions. ${ }^{36}$ Prior to my research, no research had been conducted to determine the CCC of salt with multivalent cations (see Chapter 4).

\subsection{SERS for Drug Detection}

In recent years, there has been a significant increase in analytical and forensic applications of Raman spectroscopy. The development of portable Raman spectrometers that are light, easy to carry, and produce fast results has accelerated the trend. The various forensic applications include questioned document examination, explosives/gunshot residue, trace evidence, DNA, and drug analysis. Within questioned documents, SERS analysis has been conducted on pen inks, printer inks and toners. ${ }^{37,38}$ Silver colloids have been used to differentiate between various black and blue gel and liquid pens ${ }^{37}$ and to differentiate between four different jet ink dyes printed on five different types of paper. ${ }^{38}$

In regard to trace analysis, studies were conducted on fibers and paints to detect various pigments on dyes within the various forms of art. As a consequence of background interferences, silver colloids could not be used to detect pigments and dyes on fibers. Instead, a silver paste was developed to detect various pigments and dyes on an art piece by Mariano Fortuny. ${ }^{39}$ Gold nanoparticles on silicon or carbon coated surfaces, which formed monolayers of linear arrays, were able to detect RDX and ammonium nitrate. ${ }^{40}$ In addition, research has been conducted combining Polymerase Chain Reaction (PCR) with SERS to detect specific DNA and RNA sequences using silver colloids printed on filter paper and applied to a region on a dipstick. ${ }^{41}$ 
Multiple articles have been produced showing the ability to detect various drugs via SERS. Silver nanoparticles have been used to detect morphine, codeine and hydrocodone in trace quantities with $\mathrm{NaCl}$ as an aggregating agent. ${ }^{42}$ The spectra produced provided enough spectral information to differentiate these three structurally similar molecules as well as providing assignment to how the molecules interact with the nanoparticles. Yet, the detection limits were not determined, and spectra were produced with the addition of one microgram of the drug of interest. ${ }^{42}$

Surface enhanced Raman spectroscopy has also been applied to drugs commonly found in drug facilitated sexual assaults (DFSA). In previous work by Cîntã et al., the analysis of diazepam and nitrazepam was performed on silver colloid surfaces in aqueous solution. ${ }^{43}$ The nanoparticles were synthesized using borohydride reduction. The drugs were detected and identified at a concentration of approximately $30 \mathrm{ng} / \mathrm{mL}$ with excitation wavelength of $488 \mathrm{~nm}$. Though the structures are very similar of these two compounds, they could easily be distinguished based on differences in Raman vibrational frequencies. $^{43}$

In a recent study, 11 different benzodiazepines were studied using gold nanoparticles and four different aggregating agents $\left(\mathrm{MgCl}_{2}, \mathrm{CaCl}_{2}, \mathrm{KCl}\right.$, and $\left.\mathrm{NaCl}\right) .{ }^{46}$ The gold nanoparticles were prepared using the reduction of hydrogen tetrachloroaurate with a $1 \%$ sodium citrate solution. The limits of the detection of the various benzodiazepines were determined to be $50 \mathrm{ng} / \mathrm{ml}$ or lower with an excitation wavelength of $785 \mathrm{~nm}$. On the basis of the results, the best aggregating agent to use was (1.67M) $\mathrm{MgCl}_{2}$. All benzodiazepines could be easily distinguished using differences in Raman vibrational frequencies at a concentration of $250 \mathrm{ng} / \mathrm{mL} .{ }^{44}$ Spiked urine samples containing 
benzodiazepines were detected at concentrations as low as $0.5 \mathrm{ng} / \mathrm{mL}$. Prior to SERS analysis, the benzodiazepines had to be extracted from the urine using supported liquid extraction (SLE). ${ }^{45}$

Only a few articles have been published on the use of SERS to detect various designer drugs. ${ }^{46-48}$ Mephedrone using SERS and a portable Raman spectrometer using gold and silver nanoparticles. However, the limit of detection for the optimal method was not determined. ${ }^{47}$ Farquharson et al. analyzed a variety of drugs in saliva, including MDMA, using SERS via a portable Raman spectrometer. On the basis of their analysis, all 14 drugs could be discriminated from each other via their Raman shift. ${ }^{48}$ Alphapyrrolidinopentiophenone ( $\alpha$-PVP) could be detected at concentrations as low as $0.01 \mathrm{mM}$ using silver nanoparticles. ${ }^{49}$ Stewart et al. looked at a variety of cathinones of which could be easily distinguished from one another. ${ }^{46}$

Prior to starting this research study, no research had been published on SERS detection of synthetic cannabinoids. Since our first publication, additional work on synthetic cannabinoids has been conducted, see Chapter 3. Silver nanoparticles have been used detect AMB-FUBINACA at concentrations as low as $0.01 \mathrm{mM} .{ }^{49}$ In addition, using a hydroxylamine phosphate silver ( $\mathrm{HPAg}$ ) colloid and $\mathrm{NaCl}$ as the aggregating agent, $5 \mathrm{~F}$ PB-22 could be detected at concentrations as low as $2.12 \times 10^{-8} \mathrm{M}^{50}$

\section{GOALS AND OBJECTIVES}

The goal of my thesis was to produce a surface enhanced Raman spectroscopy (SERS) technique that would be able to detect synthetic cannabinoids from biological samples at low physiological concentrations. The technique needed to use a rapid extraction method that, paired with SERS, could be used as an alternative to the 
traditional immunoassays. The SERS procedure would be able to detect the current synthetic cannabinoids as well as the future development of other synthetic cannabinoids and other designer drugs due to the unique Raman spectrum of each compound.

Therefore, SERS could be useful in a clinical setting to assess drug overdose as well as forensic toxicological analysis.

The study includes three main objectives. The first objective was to assess the use of SERS as a method for the screening of synthetic cannabinoids using previously tested methods. The second part of the study was to further investigate SERS enhancement in order to develop an optimal SERS method for the detection of synthetic cannabinoids. Gaining a better understanding of the nanoparticle and aggregating agent interaction would help determine the optimal aggregating agent and aggregating agent concentration needed for SERS analysis of synthetic cannabinoids. The final part of the study was to assess the use of supported liquid extraction as a technique to detect synthetic cannabinoids in simulated urine samples. Data collection and analysis involved observation of the Raman spectral analysis of each synthetic cannabinoid, calculation of analytical figures of merit, and analysis of simulated/spiked urine samples. 


\section{INITIAL ASSESSMENT OF A SERS METHOD FOR THE DETECTION OF SYNTHETIC CANNABINOINDS}

This article was published in Talanta, 164, Mostowtt T, McCord B., Surface enhanced Raman spectroscopy (SERS) as a method for the toxicological analysis of synthetic cannabinoids, 396-402, Copyright Elsevier (2017).

\subsection{Abstract}

Synthetic cannabinoids (K2, spice) present problems in forensic investigations because standard presumptive methods, such as immunoassays, are insufficiently specific for the wide range of potential target compounds. This issue can lead to problems with low sensitivity and yield false negative results. A potential solution to this problem is surface enhanced Raman spectroscopy (SERS). In this study we demonstrate the analysis of a set of structurally similar synthetic cannabinoids using SERS.

The procedure involves mixing the analyte with gold nanoparticles prepared in a solution containing alkali or alkaline earth salt solutions. The salts produce aggregation of the nanoparticles with a resultant spectral enhancement due to the formation of spectral hotspots with enhanced field effects within the aggregate. Among the salts tested, $0.0167 \mathrm{M} \mathrm{MgCl}_{2}$ produced the lowest limit of detection and best overall sensitivity. The method produces clearly distinguishable spectra for each synthetic cannabinoid with detection limits as low as $18 \mathrm{ng} / \mathrm{mL}$. Spiked urine samples were also analyzed following a cleanup procedure involving support liquid extraction. When using a portable Raman system, a higher concentration of $\mathrm{MgCl}_{2}$ was needed to produce similar a LOD. The results demonstrate that this procedure has great potential as a method for presumptive screening of synthetic cannabinoids. 


\subsection{Introduction}

The use and abuse of synthetic cannabinoids has increased significantly in recent years. Originally, these drugs were sold in retail outlets or via the internet and labeled as "not for human consumption" to avoid any possible regulation of the products by the Food and Drug Administration (FDA). In these internet or retail outlets, synthetic cannabinoids are sold as "K2" or "spice". ${ }^{51}$ With easy access, any individual of any age could purchase the drug giving easy access making them very popular.

This upsurge in popularity has led to a concomitant increase in drug seizures and emergency room visits around the world. The number of emergency room visits in the United States involving the use of synthetic cannabinoids increased from over 11,000 in 2010 to over 28,000 in $2011 .^{52}$ Over $75 \%$ of those visits involved people from the age of 12-29. ${ }^{52}$ In Europe, 36 people were hospitalized, following the abuse of MDMBCHMICA in one month of $2016 .{ }^{53}$ There have also been incidents in South Korea and other parts of the world. ${ }^{54}$

Many of these drugs are made in small, unregulated laboratories and easily shipped around the world using standard carriers. ${ }^{55}$ The response of public officials to this issue has been to make these problematic drugs illegal. Unfortunately, once a compound is listed and becomes illegal to possess, new synthetic analogs are quickly developed and distributed. The resultant plethora of new and dangerous compounds presents a continuing problem in forensic investigation and laboratory work as standard methods may not detect the new drug targets.

Although confirmatory testing of many drugs of abuse can be performed using advanced methods such as GC-MS and LC-MS, ${ }^{56,57}$ it is important to have a robust and 
reliable screening method. For toxicological investigations, the most commonly used technique is the immunoassay. ${ }^{14,58}$ As an example, Spinelli et al. performed a validation study on a commercial immunoassay system developed to target the JWH-018 Npentanoic acid metabolite. To assess the immunoassay's cross-reactivity, 73 synthetic cannabinoids (500 $\mu \mathrm{g} / \mathrm{L}$ concentration) were examined. Of these 73 compounds, 19 synthetic cannabinoids produced none or less than $1.2 \%$ cross-reactivity ${ }^{59}$ Similarly, Barnes et al. looked at the cross-reactivity of a different commercial immunoassay. Their study of 73 cannabinoids examined at a concentration of $10 \mu \mathrm{g} / \mathrm{L}$ found that only 17 of the compounds produced a cross-reactivity of $10 \%$ or higher, clearly indicating potential problems with false negatives. ${ }^{15}$ Although some immunoassays are highly sensitive for their targets and can be rapidly performed, ${ }^{14}$ current immunoassay testing of cannabinoids is very limited and the tests respond poorly (low cross reactivity) when confronted with the wide range of differing designer drug structures.

Because of these problems, new procedures involving GC-MS and LC-MS, have been developed for screening synthetic cannabinoids and other designer drugs. ${ }^{18,57,60,61}$ For GC-MS, sample preparation for this method involves an extraction of the drugs from the biological matrix and derivatization, which is necessary in order to produce volatile compounds. ${ }^{56,57}$ Both of these methods have limits of detection as low as $0.1 \mathrm{ng} / \mathrm{mL}$ and there is a wide range of samples that can be detected. ${ }^{18,57,60,61}$ Unfortunately, many hospital laboratories lack the expertise to perform this kind of complex analysis in a timely fashion, and the instrumentation is costly to run and maintain. A general immunoassay for dangerous drugs may be the only available test, resulting in false negatives when synthetic drug analogs are tested. 
Many samples left undetected by immunoassay may never be investigated further. In the study performed by Spinelli et al., over 2400 previously tested authentic case urine samples were examined. Upon analysis, 57 false negatives were produced at a cut-off of $5 \mu \mathrm{g} / \mathrm{L}$ and 87 false negatives were produced at a cut-off of $10 \mu \mathrm{g} / \mathrm{L} .{ }^{59}$ The above issue with false negatives and lack of cross-reactivity is a likely result of poor cross-reactivity between designer drug structures.

The inevitable problem with immunoassays is that the procedures targets one particular compound or structural type. The wide range of synthetic cannabinoids available virtually guarantees that screening via immunoassay will cause false negatives. An alternative procedure is needed that is flexible, specific and sensitive. For this reason, we have been investigating surface enhanced Raman spectroscopy for use in screening synthetic cannabinoids. Because immunoassay tests are class and not compound specific, even a positive result cannot provide much guidance for subsequent confirmation by GCMS or LC-MS. Instead, itis an orthogonal method that produces unique spectra for each compound. This however is not the case for Raman spectroscopy.

Unlike conventional Raman techniques, surface enhanced Raman Spectroscopy (SERS) has sufficient sensitivity to detect these drugs at low physiological conditions. The initial SERS procedure was developed by Fleischmann et al. in $1974 .^{28}$ In these early experiments, it was discovered that the Raman signal was greatly enhanced when the sample was deposited on roughened metal surfaces. The effect was believed to result from the result of electric field enhancements on the metal surfaces that accentuated interactions between adsorbed compounds and surface plasmons. Later work by Lee and 
Miesel demonstrated that similar enhancements could be created in a solution containing metallic nanoparticles. ${ }^{62}$

Recently, there has been a renewed interest in SERS as a screening tool for trace analysis. ${ }^{63,64}$ SERS has been employed for the identification of trace identification of certain controlled substances. The drugs previously examined have included amphetamines, ${ }^{27,64-68}$ opiates, ${ }^{27,65,69,70}$ benzodiazepines, ${ }^{43-45}$ and designer drugs. ${ }^{49,50}$

In previous work in this laboratory we have demonstrated the application of SERS for the detection of benzodiazepines in aqueous and toxicological samples at concentrations as low as $0.5 \mathrm{ng} / \mathrm{mL} .{ }^{44,45}$ During a study of potential interferences with this technique, we were able to determine that THC could be detected at concentrations as low as $5 \mathrm{ng} / \mathrm{mL}$. For this reason, we began to examine the SERS spectra of natural and synthetic cannabinoids. In this study, four synthetic cannabinoids were examined, JWH073, JWH-018, JWH-081, and JWH-122. The structures of these compounds are relatively similar and are shown in Figure 7. The overall goal of the project was to determine if SERS could differentiate and detect synthetic cannabinoids of a closely similar chemical structure at low $\mathrm{ng} / \mathrm{mL}$ concentrations. To our knowledge this is the first description of the application of SERS for synthetic cannabinoids.

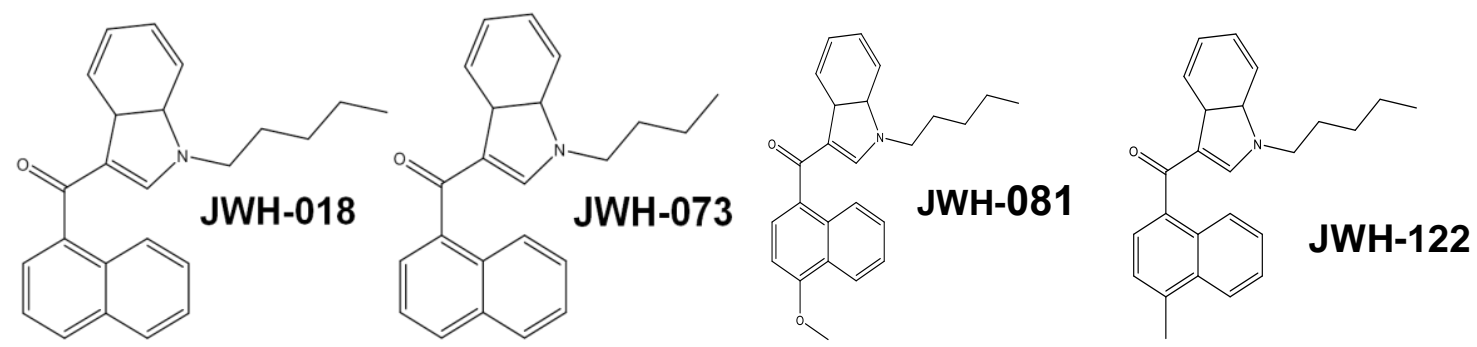

Figure 7: The chemical structure of the 4 different synthetic cannabinoids examined in this study. 


\subsection{Materials and Methods}

\subsubsection{Drugs examined}

All DEA scheduled drugs JWH-018 (LOT\# 1294.1B3.2), JWH-073 (LOT\#

1293.1B1.2), JWH-081 (LOT\# 1505.1B1.1), and JWH-122 (LOT\# 1491.1B1.1) were obtained from Lipomed AG. All of the DEA scheduled drugs were obtained in powder form and diluted to $0.2 \mathrm{mg} / \mathrm{mL}$ in methanol. The above solution was then used to create stock standard solutions ranging from 25 to $20,000 \mathrm{ng} / \mathrm{mL}$ in $10 \%$ methanol.

\subsubsection{Aggregating agent optimization}

The following procedures used for SERS development and analysis were similar to those followed by Doctor et al. ${ }^{44} 100 \mathrm{~mL}$ gold nanoparticle solutions were prepared reducing tetrachloroauric (III) acid trihydrate (Acros Organics, LOT\#A0354619) with 4 $\mathrm{mL}$ of a $1 \%$ by weight solution of trisodium citrate dihydrate (Fisher Scientific, LOT\# 091236). Chlorates, nitrates, and sulfates were used as aggregating agents to help determine the optimal aggregating agent and concentration used for SERS analysis. The previous study by Doctor et al. only looked at chloride salts as aggregating agents. Initially, $1.67 \mathrm{M}$ stock solutions of $\mathrm{MgCl}_{2}$, (Fisher Scientific), $\mathrm{CaCl}_{2}$ (Fisher Scientific), $\mathrm{KCl}$ (Spectrum), $\mathrm{NaCl}$ (Sigma-Aldrich), $\mathrm{MgSO}_{4}$ (Fisher Scientific), $\mathrm{Na}_{2} \mathrm{SO}_{4}$ (Spectrum), and $\mathrm{KNO}_{3}$ (Sigma-Aldrich) were examined in SERS analysis. Based upon initial results, further analysis was examined using stock concentrations of $0.835 \mathrm{M}, 1.25 \mathrm{M}, 2.5 \mathrm{M}$ and 3.34 $\mathrm{M}$ of $\mathrm{MgCl}_{2}$. After the preparation of the above solutions, the SERS samples for analysis were prepared as follows. First, $980 \mu \mathrm{L}$ of the gold nanoparticle solution was placed in a clear $2 \mathrm{~mL}$ sample vial (Fisher Scientific) followed by $10 \mu \mathrm{L}$ of a select aggregating agent solution and vortexed for $30 \mathrm{~s}$. Then, $10 \mu \mathrm{L}$ of the drug stock standard 
solution was added to the $2 \mathrm{~mL}$ sample vial and vortexed for $30 \mathrm{~s}$. The final concentration of each solution, in the gold nanoparticle solution was as follows: $0.05 \mathrm{mM} \mathrm{HAuCl}_{4}$, $0.0835-0.334 \mathrm{M}$ of aggregating agent, and between $2.5-2000 \mathrm{ng} / \mathrm{mL}$ of a synthetic cannabinoid.

\subsubsection{Simulated urine samples}

Upon the optimization of the above method, spiked urine samples of JWH-018, JWH-073, JWH-081, and JWH-122 in pure methanol were analyzed using supported liquid extraction (SLE). For sample pretreatment, a 1:1 solution of spiked urine sample: water and spiked urine sample: $100 \mathrm{mM} \mathrm{NH} \mathrm{H}_{4} \mathrm{OAc}$ buffer solution (adjusted to $\mathrm{pH} 4$ with $1 \%$ formic acid) were examined (total solution volume of $2 \mathrm{~mL}$ ) and applied to the Biotage Isolute supported liquid extraction (SLE) $+2 \mathrm{~mL}$ column under vacuum and let to sit for $5 \mathrm{~min}$. Next, two washes of $2 \mathrm{~mL}$ dichloromethane (Acros Organics) under gravity were applied to the SLE+ column and let sit for two minutes after each wash. Then, a vacuum was applied to the SLE+ column eluting the dichloromethane, which was dried down under nitrogen gas and reconstituted with $10 \%$ methanol. Finally, $10 \mu \mathrm{L}$ of this urine extracted sample was used into the formation of the SERS solution mentioned in the above paragraph for analysis.

\subsubsection{Instrumentation}

The gold nanoparticles were characterized via ultraviolet-visible absorbance (Varian Cary 100 Bio UV-visible spectrophotometer) and dynamic light scattering (Malvern Zetasizer Nano). In addition to the nanoparticle size, the zeta potential of the gold nanoparticles was measured using a Malvern Zetasizer Nano. The SERS solution was transferred to a quartz suprasil cuvette (Hellma Analytics) and analyzed using a 
Perkin Elmer Raman Station 400F (bench top model) with a $785 \mathrm{~nm}$ laser at $100 \mathrm{~mW}$ power with a resolution of $4 \mathrm{~cm}^{-1}$ (FWHM) for 5 scans with $4 \mathrm{~s}$ of laser exposure or to a Thermo Scientific First Defender RM (portable) with a $785 \mathrm{~nm}$ laser at high power (250 $\mathrm{mW}$ ) and a resolution of $7-10.5 \mathrm{~cm}^{-1}(\mathrm{FWHM})$. All samples were run in triplicate and used to calculate limit of detection.

\subsection{Results and discussion}

In this study, four structurally similar JWH synthetic cannabinoids (JWH-018, JWH-073, JWH-081, and JWH-122) were examined. The purpose of this work was to determine if SERS was capable of differentiation of 4 structurally similar synthetic cannabinoids at toxicological concentrations. This work is divided into four parts. Part one focused on the gold nanoparticle synthesis used for SERS analysis. Part two focused on the evaluation of chloride, sulfate, and nitrate salts to determine the optimal aggregating agent concentration to detect the four synthetic cannabinoids examined. Part

three assessed the use of a portable Raman spectrometer in our SERS method, comparing to our bench top results from part two. Part four looked into the use of SERS for the toxicological screening of spiked urine samples. To our knowledge, this is the first report in the literature involving SERS analysis of these compounds.

\subsubsection{Gold nanoparticle synthesis}

The gold nanoparticles used in this study were created using a citrate reduction method similar to Doctor et al. ${ }^{44}$ The reaction was deemed complete when the tetrachloroauric (III) acid trihydrate solution changed from a yellow to a final wine red color following the addition of the sodium citrate. The resultant nanoparticles were spherical in shape and ranged in size from $10 \mathrm{~nm}$ to $100 \mathrm{~nm}$ in diameter with an average 
size of $24.5 \pm 4.7 \mathrm{~nm}$, and a zeta potential of $-32.5 \pm 1.5 \mathrm{mV}$. The UV-VIS peak

absorbance was $521 \pm 1.7 \mathrm{~nm}$ (Figure $8 \mathrm{a}-\mathrm{c})$. The peak absorption and size distribution was similar to previous results obtained by Doctor et $a l .{ }^{45}$ and size distribution was similar to previous results obtained by Doctor et al. ${ }^{44}$

(a)

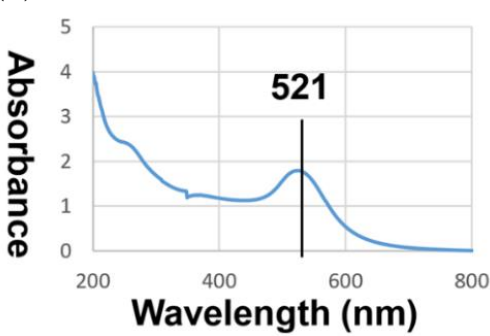

(b)

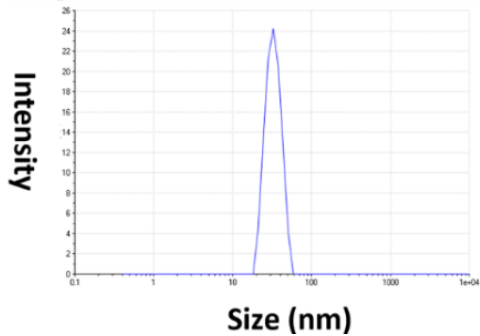

(c)

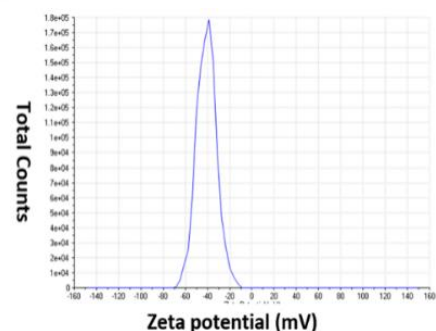

Figure 8: Characterization of synthesized nanoparticles (a) UV-VIS absorption spectra; (b) size distribution; (c) zeta potential distribution

\subsubsection{Aggregating agent evaluation}

In the previous work in this laboratory, chloride salts at various concentrations were examined to determine the optimal method for gold nanoparticle aggregation and SERS detection of benzodiazepines. Overall it was determined that a concentration of $0.0167 \mathrm{M} \mathrm{MgCl}_{2}$ produced the optimal sensitivity. ${ }^{44}$ In this work, a similar set of chlorate salts as well as different salts, such as sulfates and a nitrate, were examined to determine their effect on nanoparticle aggregation and its associated enhancements to the Raman signal for the cannabinoids.

The cannabinoid samples were prepared for analysis as follows: $980 \mu \mathrm{L}$ of the nanoparticle solution was mixed with $10 \mu \mathrm{L}$ of a select aggregating agent stock solution and vortexed for $30 \mathrm{~s}$. Next, $10 \mu \mathrm{L}$ of the drug stock standard solution was added and vortexed for $30 \mathrm{~s}$, then placed in a cuvette for analysis by the Raman spectrometer. The final concentration of the analyzed samples were as follows: $0.05 \mathrm{mM} \mathrm{HAuCl}_{4}, 0.0167 \mathrm{M}$ 
of aggregating agent, and between $2.5-2000 \mathrm{ng} / \mathrm{mL}$ of the synthetic cannabinoid. Seven different aggregating agents were examined including $\mathrm{MgCl}_{2}, \mathrm{CaCl}_{2}, \mathrm{NaCl}$, and $\mathrm{KCl}$ as well as $\mathrm{MgSO}_{4}, \mathrm{Na}_{2} \mathrm{SO}_{4}$ and $\mathrm{KNO}_{3}$. The aggregating agent concentration was set at $0.0167 \mathrm{M}$ due to results of optimized concentrations from previous studies and to limit the amount of variables at the start of this study. Of the seven aggregating agents, only $\mathrm{MgCl}_{2}, \mathrm{CaCl}_{2}$, and $\mathrm{MgSO}_{2}$ produced nanoparticle aggregation (a change from a red to a grey solution) with subsequent Raman enhancement at this concentration (Figure 9).

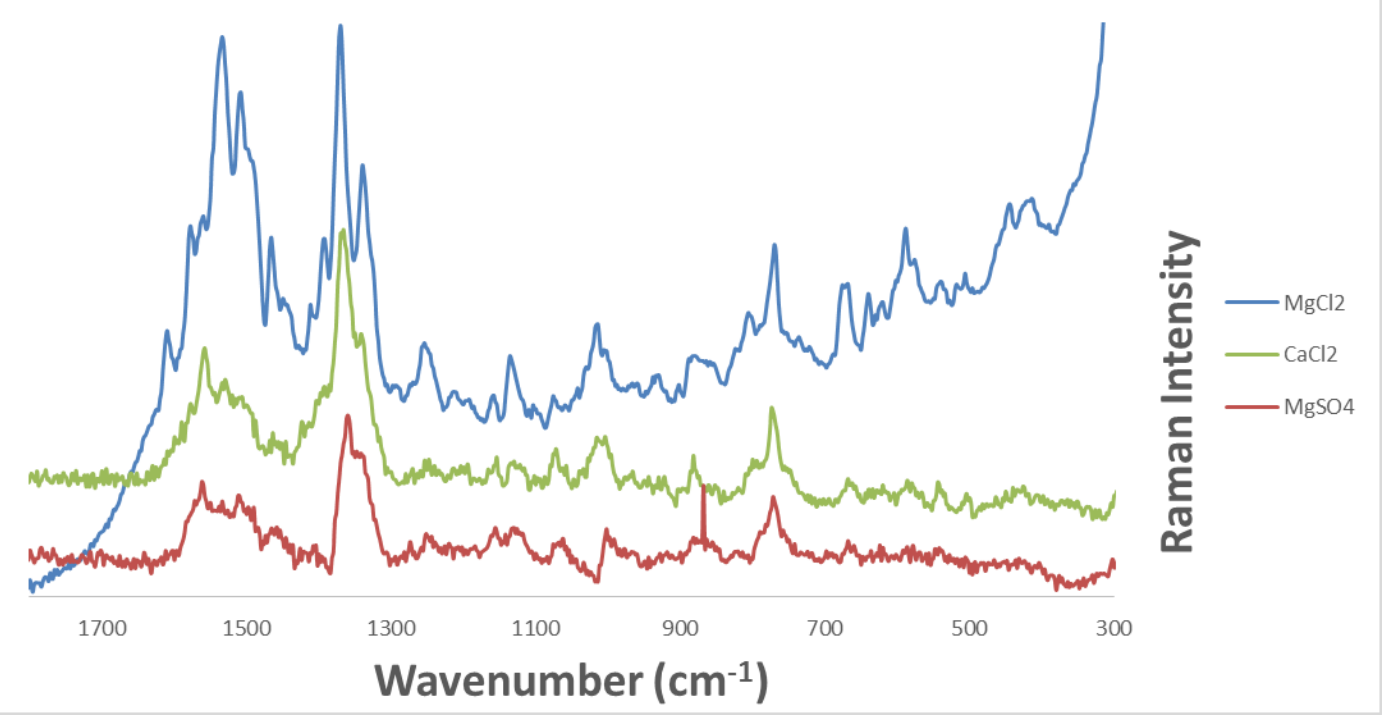

Figure 9: Raman spectra of JWH-073 via the addition of $\mathrm{MgCl}_{2}$ (top), $\mathrm{CaCl}_{2}$ (middle) and $\mathrm{MgSO}_{4}$ (bottom) at $250 \mathrm{ng} / \mathrm{mL}$.

Figure 7 shows the Raman spectrum of each compound with $0.0167 \mathrm{M} \mathrm{MgCl}_{2}$ as the aggregating agent. A prominent peak in each spectrum was used to establish the slope, correlation coefficient, and limit of detection (LOD) for each compound. Compared to $\mathrm{CaCl}_{2}$ and $\mathrm{MgSO}_{4}, \mathrm{MgCl}_{2}$ produced the greatest sensitivity and lowest LOD for all four synthetic cannabinoids (Table 1). For three out of the four JWH compounds, $\mathrm{MgCl}_{2}$ produced more than a tenfold lower limit of detection. Based upon the above parameters, it was determined that the best aggregating agent to use was $\mathrm{MgCl}_{2}$. 
It is notable that each compound produced a unique and identifiable Raman spectrum that could be distinguished from the others (Figure 10). JWH-018 and JWH073 are similar in chemical structure, only varying in the length of the alkyl chain off the nitrogen and have similar Raman spectrum. Yet, there are a few differences. Firstly, JWH- 018 has an additional peak at $700 \mathrm{~cm}^{-1}$ not seen in JWH-073. Second, there are differences in the peak locations near $1396 \mathrm{~cm}^{-1}$ and $1574 \mathrm{~cm}^{-1}$ producing a shift of $4 \mathrm{~cm}^{-1}$ (to $1392 \mathrm{~cm}^{-1}$ and $1578 \mathrm{~cm}^{-1}$ ) in the Raman spectra of JWH-018. Lastly, JWH-073 contains additional spectral peaks at and below $650 \mathrm{~cm}^{-1}$.
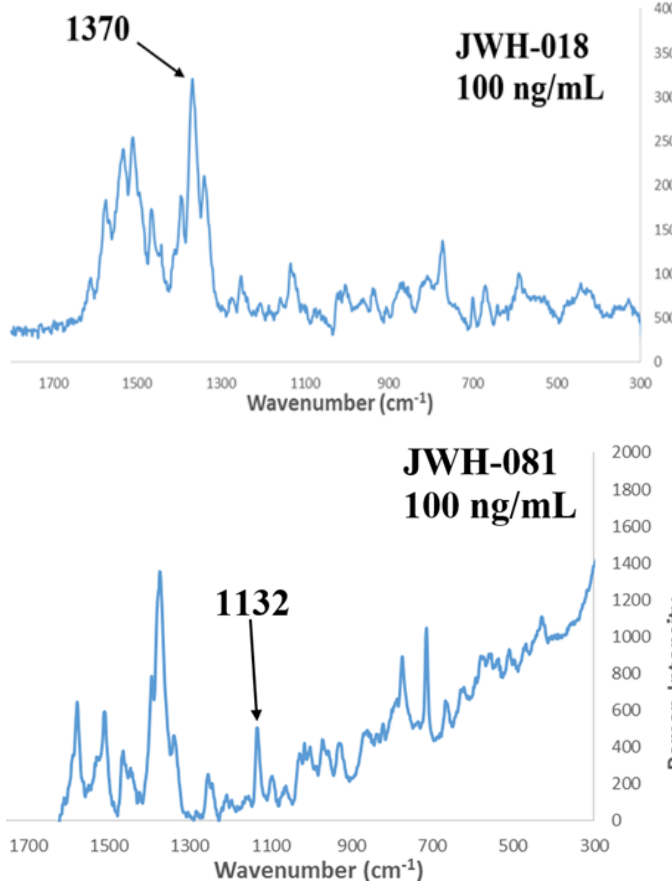

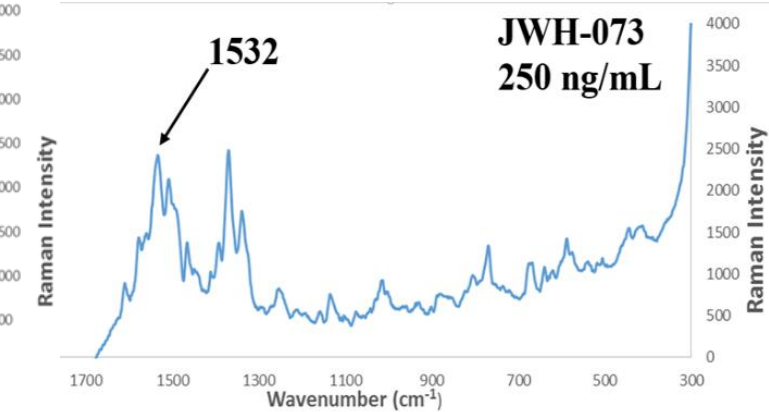

JWH-122 1900 $100 \mathrm{ng} / \mathrm{mL}$

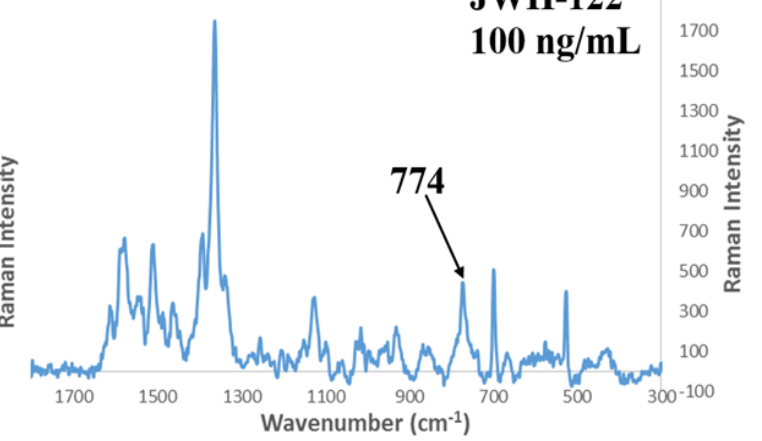

Figure 10: The Raman spectrum produced from JWH-018, JWH-073, JWH-081, and $\mathrm{JWH}-122$ using $0.0167 \mathrm{M} \mathrm{MgCl}_{2}$. The indicated peak was used to determine the LOD of each compound. 


\begin{tabular}{|c|c|c|c|c|c|c|}
\hline Drug & $\begin{array}{l}\text { Aggregating } \\
\text { agent }\end{array}$ & $\begin{array}{c}\text { Dynamic } \\
\text { Range } \\
(\mathrm{ng} / \mathrm{mL})\end{array}$ & $\begin{array}{c}\text { Sensitivity } \\
\text { (Intensity/ } \\
\text { Concentration) }\end{array}$ & $\mathrm{R}^{2}$ & $\begin{array}{l}\text { Standard } \\
\text { Deviation }\end{array}$ & $\begin{array}{c}\text { Calculated } \\
\text { LOD } \\
(\mathrm{ng} / \mathrm{ml})\end{array}$ \\
\hline \multirow{3}{*}{$\begin{array}{c}\text { JWH- } \\
018\end{array}$} & $\mathrm{MgCl}_{2}$ & $2.5-250$ & 31 & 0.99 & $5.3 \times 10^{2}$ & 51 \\
\hline & $\mathrm{CaCl}_{2}$ & $25-500$ & 8.7 & 0.92 & $2.4 \times 10^{2}$ & 84 \\
\hline & $\mathrm{MgSO}_{4}$ & $2.5-500$ & 2.3 & 0.96 & $2.2 \times 10^{2}$ & $2.8 \times 10^{2}$ \\
\hline \multirow{3}{*}{$\begin{array}{l}\text { JWH- } \\
073\end{array}$} & $\mathrm{MgCl}_{2}$ & $25-500$ & 9.6 & 0.99 & $1.8 \times 10^{2}$ & 56 \\
\hline & $\mathrm{CaCl}_{2}$ & $25-250$ & 1.8 & 0.73 & $1.5 \times 10^{2}$ & $2.5 \times 10^{2}$ \\
\hline & $\mathrm{MgSO}_{4}$ & $25-1000$ & 0.36 & 0.91 & $1.2 \times 10^{2}$ & $9.9 \times 10^{2}$ \\
\hline \multirow{3}{*}{$\begin{array}{c}\text { JWH- } \\
081\end{array}$} & $\mathrm{MgCl}_{2}$ & $2.5-250$ & 2.9 & 0.90 & $1.3 \times 10^{3}$ & 18 \\
\hline & $\mathrm{CaCl}_{2}$ & $100-1000$ & 0.5 & 0.98 & 64 & $3.8 \times 10^{2}$ \\
\hline & $\mathrm{MgSO}_{4}$ & $2.5-1000$ & 0.24 & 0.95 & 61 & $7.7 \times 10^{2}$ \\
\hline \multirow{3}{*}{$\begin{array}{l}\text { JWH- } \\
122\end{array}$} & $\mathrm{MgCl}_{2}$ & $2.5-250$ & 3.4 & 0.99 & 68 & 60 \\
\hline & $\mathrm{CaCl}_{2}$ & $25-500$ & 2.5 & 0.99 & $2.0 \times 10^{2}$ & $2.3 \times 10^{2}$ \\
\hline & $\mathrm{MgSO}_{4}$ & $25-500$ & 0.05 & 0.22 & 72 & $4.3 \times 10^{3}$ \\
\hline
\end{tabular}

Table 1: A comparison of the effect of $0.0167 \mathrm{M} \mathrm{MgCl}_{2}, \mathrm{CaCl}_{2}$, and $\mathrm{MgSO}_{4}$ when used as aggregating agents for the detection of JWH-018, JWH-073, JWH-081, and JWH-122.

\subsection{3. $\mathrm{MgCl}_{2}$ optimization}

Further analysis was used to determine the effect of variation in the $\mathrm{MgCl}_{2}$

concentration on sensitivity (Table 2). Three different concentrations of $\mathrm{MgCl}_{2}$ were examined for use as aggregating agents: $0.00835 \mathrm{M}, 0.00167 \mathrm{M}$, and $0.0334 \mathrm{M}$. These solutions were combined with $2.5-2000 \mathrm{ng} / \mathrm{mL}$ of each synthetic cannabinoid and analyzed. To determine the LOD, the same spectral peaks used for part 1 were used for part 2. For all four compounds, the $0.0167 \mathrm{M}$ concentration produced the highest slope, best linear correlation (R2 value), and lowest LOD. 


\begin{tabular}{|c|c|c|c|c|c|c|}
\hline Drug & $\begin{array}{c}\mathrm{MgCl}_{2} \\
\text { Concentration } \\
(\mathrm{M})\end{array}$ & $\begin{array}{c}\text { Dynamic } \\
\text { Range } \\
(\mathrm{ng} / \mathrm{mL})\end{array}$ & $\begin{array}{c}\text { Sensitivity } \\
\text { (Intensity/ } \\
\text { Concentration) }\end{array}$ & $\mathrm{R}^{2}$ & $\begin{array}{c}\text { Standard } \\
\text { Deviation }\end{array}$ & $\begin{array}{c}\text { Calculated } \\
\text { LOD } \\
(\mathrm{ng} / \mathrm{ml})\end{array}$ \\
\hline $\begin{array}{c}\text { JWH- } \\
018\end{array}$ & 0.00835 & $25-500$ & 11 & 0.88 & $2.5 \times 10^{2}$ & 72 \\
\hline JWH- & 0.0167 & $2.5-250$ & 31 & 0.99 & $5.3 \times 10^{2}$ & 51 \\
\hline 073 & 0.0334 & $25-500$ & 3.6 & 0.97 & $3.3 \times 10^{2}$ & $2.8 \times 10^{2}$ \\
\hline \multirow{2}{*}{$\begin{array}{c}\text { JWH- } \\
081\end{array}$} & 0.0167 & $25-500$ & 9.6 & 0.99 & $1.8 \times 10^{2}$ & 56 \\
\hline & 0.0334 & $2.5-1000$ & 1.9 & 0.97 & 88 & $1.4 \times 10^{2}$ \\
\hline JWH- & 0.00835 & $25-500$ & 5.1 & 0.97 & $2.1 \times 10^{2}$ & $1.3 \times 10^{2}$ \\
\hline 122 & 0.0334 & $2.5-250$ & 2.9 & 0.90 & $1.3 \times 10^{3}$ & 18 \\
\hline & 0.00835 & $2.5-250$ & 3.8 & 0.95 & $1.1 \times 10^{2}$ & 90 \\
\hline & 0.0334 & $25-500$ & 0.65 & 0.92 & 35 & $1.6 \times 10^{2}$ \\
\hline
\end{tabular}

Table 2: A comparison of $0.00835 \mathrm{M}, 0.0167 \mathrm{M}$, and $0.0334 \mathrm{M} \mathrm{MgCl}_{2}$ used to determine the optimal aggregating agent concentration for the detection of JWH-018, JWH-073, JWH-081, and JWH-122.

\subsubsection{Bench top Raman vs. portable Raman comparison}

After the completion of optimization of the SERS method for the detection of four different synthetic cannabinoid compounds, a second analysis was preformed using a portable Raman spectrometer to determine if similar results could be obtained. There are a few differences between the two Raman instruments, such laser power, instrument resolution, and scan settings varied. The Perkin Elmer Raman (bench top model) has a $100 \mathrm{~mW}$ laser with a resolution of $4 \mathrm{~cm}^{-1}$ (FWHM) while the Thermo Scientific First Defender RM (portable) used a laser at a high power setting of $250 \mathrm{~mW}$ with a resolution of $7-10.5 \mathrm{~cm}^{-1}$ (FWHM). For the benchtop system, each sample was scanned 5 times with $4 \mathrm{~s}$ exposure time, while for the portable system, the number of scans and the exposure time was varied based on input signal. The results from the portable Raman spectrometer is shown Table 3 . In addition to the 3 different $\mathrm{MgCl}_{2}$ concentrations 
previously tested on the bench top model, $0.0125 \mathrm{M}$ and $0.025 \mathrm{M} \mathrm{MgCl}_{2}$ were also examined to further determine the optimal $\mathrm{MgCl}_{2}$ concentration for our SERS method.

The concentration of the gold nanoparticle solution and the synthetic cannabinoid concentrations were the same as before.

\begin{tabular}{|c|c|c|c|c|c|c|}
\hline Drug & $\begin{array}{c}\mathrm{MgCl}_{2} \\
\text { Concentration }\end{array}$ & $\begin{array}{c}\text { Dynamic } \\
\text { Range } \\
\text { (ng/mL) }\end{array}$ & $\begin{array}{c}\text { Sensitivity } \\
\text { (Intensity/ } \\
\text { Concentration) }\end{array}$ & $\mathrm{R}^{2}$ & $\begin{array}{c}\text { Standard } \\
\text { Deviation }\end{array}$ & $\begin{array}{c}\text { Calculated } \\
\text { LOD } \\
(\mathrm{ng} / \mathrm{ml})\end{array}$ \\
\hline \multirow{3}{*}{$\begin{array}{c}\text { JWH- } \\
018\end{array}$} & 0.00835 & $2.5-100$ & 0.013 & 0.86 & 0.027 & 63 \\
\cline { 2 - 7 } & 0.0125 & $2.5-100$ & 0.0009 & 0.92 & 0.0007 & 18 \\
\cline { 2 - 7 } & 0.025 & $2.5-250$ & 0.003 & 0.97 & 0.026 & $2.6 \times 10^{2}$ \\
\cline { 2 - 7 } & 0.0334 & $2.5-250$ & 0.0002 & 0.94 & 0.024 & $3.6 \times 10^{2}$ \\
\hline \multirow{3}{*}{ JWH- } & 0.00835 & $25-500$ & 0.005 & 0.86 & 0.006 & 34 \\
\cline { 2 - 7 } & 0.0125 & $2.5-100$ & 0.0003 & 0.87 & 0.01 & $1.0 \times 10^{2}$ \\
\cline { 2 - 7 } & 0.0167 & $25-500$ & 0.00005 & 0.98 & 0.012 & $7.1 \times 10^{2}$ \\
\cline { 2 - 7 } & 0.025 & $2.5-250$ & 0.0001 & 0.79 & 0.012 & $3.5 \times 10^{2}$ \\
\hline \multirow{3}{*}{ JWH- } & 0.0334 & $2.5-250$ & 0.0007 & 0.94 & 0.012 & 51 \\
\cline { 2 - 7 } & 0.00835 & $2.5-500$ & 0.0001 & 0.97 & 0.036 & $1.1 \times 10^{3}$ \\
\cline { 2 - 7 } & 0.0125 & $25-250$ & 0.0002 & 0.85 & 0.087 & $1.3 \times 10^{3}$ \\
\cline { 2 - 7 } & 0.0167 & $2.5-500$ & 0.0002 & 0.89 & 0.047 & $7.0 \times 10^{2}$ \\
\cline { 2 - 7 } & 0.025 & $25-250$ & 0.0002 & 0.99 & 0.047 & $7.1 \times 10^{2}$ \\
\hline \multirow{3}{*}{ JWH- } & 0.0334 & $2.5-500$ & 0.0002 & 0.88 & 0.002 & 34 \\
\cline { 2 - 7 } 122 & 0.00835 & $10-250$ & 0.0002 & 0.88 & 0.022 & $3.4 \times 10^{2}$ \\
\cline { 2 - 7 } & 0.0167 & $5-250$ & 0.0002 & 0.72 & 0.047 & $7.0 \times 10^{2}$ \\
\cline { 2 - 7 } & 0.025 & $2.5-100$ & 0.0003 & 0.93 & 0.0066 & 66 \\
\cline { 2 - 7 } & 0.0334 & $2.5-100$ & 0.0003 & 0.78 & 0.0042 & 42 \\
\hline
\end{tabular}

Table 3: A comparison of $0.00835 \mathrm{M}, 0.0125 \mathrm{M}, 0.0167 \mathrm{M}, 0.025 \mathrm{M}$ and $0.0334 \mathrm{M} \mathrm{MgCl}_{2}$ used to determine the optimal aggregating agent concentration for the detection of JWH018, JWH-073, JWH-081, and JWH-122 using the portable Raman spectrometer.

Upon analysis of the portable Raman data, the optimal concentration of $\mathrm{MgCl}_{2}$ aggregation used in the Raman analysis of each JWH compound varied. However, for 3 out of the $4 \mathrm{JWH}$ synthetic cannabinoids, a concentration higher than $0.0167 \mathrm{M} \mathrm{MgCl}_{2}$ produced the lowest calculated LOD, with the exception that $0.0125 \mathrm{M}$ was optimal for 
JWH-018. For JWH-073 and JWH-081, the $0.0334 \mathrm{M} \mathrm{MgCl}_{2}$ produced the lowest sensitivity and calculated LOD. These results may simply be the result of differences in optical configurations and laser power. Therefore, we suggest that users should optimize nanoparticle and aggregating agent concentrations when using different instrument configurations.

To further assess the capability of the portable Raman system, a comparison of the library spectrum of the JWH-018, JWH-073, JWH081, and JWH-122 was made with the SERS spectrum obtained from that same instrument (Figure 11). The main differences in the library spectra are seen near $500 \mathrm{~cm}^{-1}$ and in between 1500 and $1800 \mathrm{~cm}^{-1}$. JWH018 and JWH-073 are nearly identical but can be resolved based upon the additional peak seen at $1288 \mathrm{~cm}^{-1}$ and double peaks near $1600 \mathrm{~cm}^{-1}$ seen in the JWH-073 spectrum. For JWH-081 and JWH-122 the bench top, portable, and library spectra are similar. For JWH-018 and JWH-073 benchtop spectra varies slightly between the portable and library spectra in peak intensity and resolution. However, for all compounds analyzed with the portable Raman, the library search feature identified the correct compound, indicating the specificity of the analysis and also demonstrating that obtained SERS spectra for these compounds is very similar to that obtained from drug powders. 


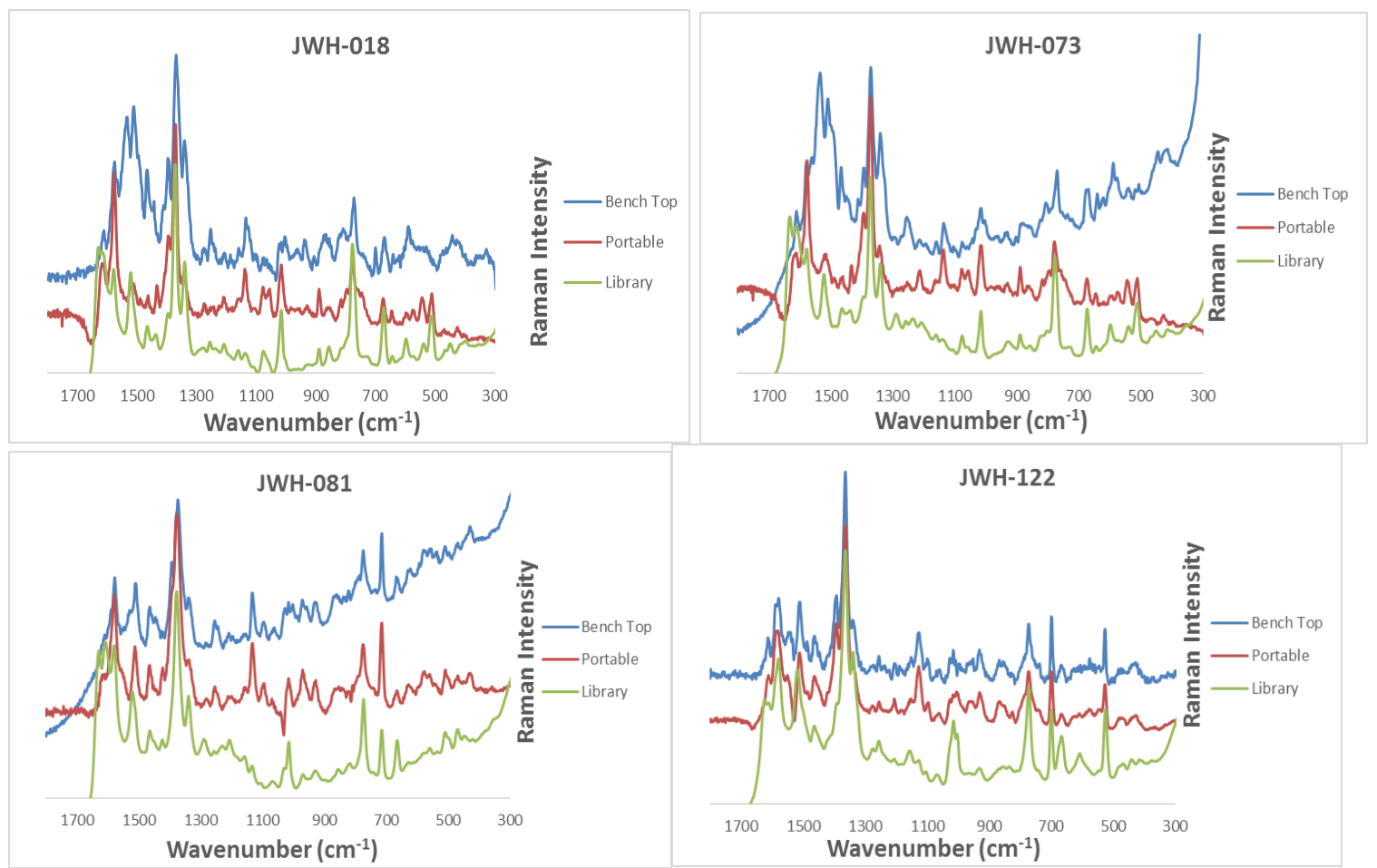

Figure 11: A comparison of the benchtop (top), portable (middle), and library (bottom) Raman spectra of JWH-018 (100 ng/mL), JWH-073 (250 ng/mL), JWH-081 (100 $\mathrm{ng} / \mathrm{mL})$, and JWH-122 (100 ng/mL) with $0.0167 \mathrm{M} \mathrm{MgCl}_{2}$ as an aggregating agent.

\subsubsection{Spiked urine sample}

To examine the capability to the SERS method to screen biological samples, an analysis was performed on a set of spiked urine samples containing JWH-018, JWH-073, JWH-081, or JWH-122 using supported liquid extraction (SLE). SLE is a quick extraction method that takes under $20 \mathrm{~min}$ and uses a silica based solid support to caputure the aqueous phase, while still permitting later transfer into an organic solvent. ${ }^{45}$ For this analysis, urine samples were diluted 1:1 with water or diluted 1:1 with buffer (100 mM NH4OAc buffer solution adjusted to $\mathrm{pH} 4$ with $1 \%$ formic acid). After dilution, the urine column. Two 5 min washes of dichloromethane were performed and the sample was eluted under vacuum. This solution was then evaporated to dryness under nitrogen gas and reconstituted with $10 \%$ methanol into $4 \mathrm{~mL}$ total volume. Then, $10 \mu \mathrm{L}$ of 
extracted sample was added to the sample analysis solution containing: $0.05 \mathrm{mM} \mathrm{HAuCl}_{4}$ and $0.0334 \mathrm{M}$ of aggregating agent.

A comparison of the Raman spectra of JWH-018 obtained involving urine samples analyzed after or without extraction prior to analysis (Figure 12). All spiked urine samples were analyzed at the concentration of $500 \mathrm{ng} / \mathrm{mL}$. These samples were analyzed with the First Defender RM portable Raman system. Based these results, only urine samples diluted with water produced a usable spectra. The recovery of JWH-018 was $86 \%$. This result demonstrates that the SLE can be used as an extraction method for the detection of JWH synthetic cannabinoids.

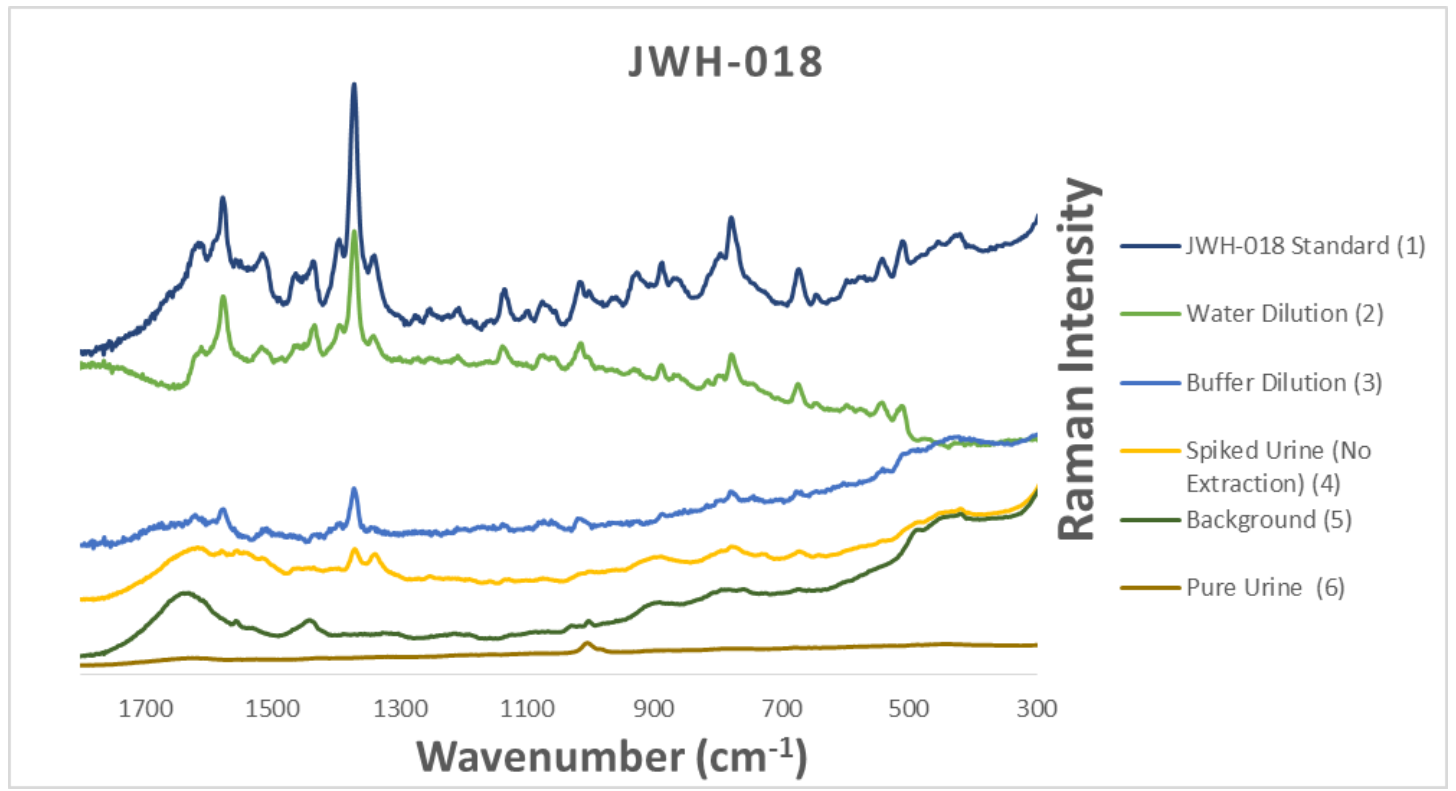

Figure 12: The Raman spectrum of a JWH-018 standard (1000 ng/mL) using from the optimized SERS method (1), a spiked urine sample $(500 \mathrm{ng} / \mathrm{mL})$ with 1:1 water dilution (2), spiked urine sample (500 $\mathrm{ng} / \mathrm{mL})$ with 1:1 ammonium acetate buffer dilution (3), spiked urine sample (2000 ng/mL) without any extraction (yellow), along with background sample (4) and pure urine (5).

\subsection{Conclusion}

This paper demonstrates the application of surface enhanced Raman spectroscopy in the detection of synthetic cannabinoids. An analysis of chloride, sulfate, and nitrate 
salts as aggregating agents were conducted. The optimal SERS procedure involves the mixing of an unknown sample with gold nanoparticles and $0.0167 \mathrm{M} \mathrm{MgCl}_{2}$ as an aggregating agent. This new procedure can easily distinguish 4 structurally similar cannabinoids with LODs ranging from 18 to $60 \mathrm{ng} / \mathrm{mL}$. For determination of these compounds in urine, a quick extraction method using supported liquid extraction was sufficient for detection. Overall this method is quick, sensitive and can be used in combination with portable instrumentation. Unlike standard immunoassays, the procedure provides identifiable spectra which can assist the analyst in downstream confirmation by mass spectrometry. It should prove to be a powerful tool for screening toxicological samples. 


\title{
4. AN EVALUATION OF MONOVALENT, DIVALENT, AND TRIVALENT
}

\section{CATIONS FOR THE SERS ANALYSIS OF SYNTHETIC CANNABINOIDS}

\author{
Reproduced from Mostowtt T, Munoz J, McCord B. An evaluation of monovalent,
} divalent, and trivalent cations as aggregating agents for surface enhanced Raman spectroscopy (SERS) analysis of synthetic cannabinoids. Analyst. 2019 Mar 1;164: 396402 with permission from the Royal Society of Chemistry.

\subsection{Abstract}

In this study monovalent, divalent, and trivalent chloride, sulfate and nitrate salts were examined to determine the critical coagulation concentration (CCC) for each salt and its corresponding effect on detection limits for SERS analysis of cannabinoid extracts. The CCC of each salt was determined using UV-vis, particle size and zeta potential measurements. The results demonstrated that the divalent and trivalent cations produce a lower CCC when compared to monovalent cations. The data indicate the effect that cation valence has on nanoparticle destabilization. This information is essential because it permits the user to develop optimal conditions for aggregation of salts used in SERS. Tests were then performed to determine the best conditions for SERS detection of a small set of cannabinoids with naphthyl indole moieties. The results demonstrate the importance of salt concentration for colloid aggregation and illustrate the effect of aggregation on sensitivity for the SERS experiment.

\subsection{Introduction}

A critical feature of surface enhanced Raman spectroscopy (SERS) is the use of a metal substrate, which can be in the form of roughened metal surfaces or colloidal metal nanoparticles, typically produced from coinage metals. A variety of methods have been 
developed to create nanocolloids in various shapes including spheres, rods, and stars, using several different reducing agents. One of the most common methods of producing gold nanoparticles utilizes sodium citrate as the reducing agent for gold chloride salts. A significant amount of research has been conducted to develop theories on the nucleation, growth, and formation of stable, polydisperse gold nanoparticles. ${ }^{29,31,71-73}$

For optimal SERS enhancement, it is common to add a chloride salt to the colloidal metal nanoparticle solution. This destabilizes the nanoparticles creating 'hot spots' which provide signal enhancement due to localized enhancement of the electric field. ${ }^{25}$ Multiple studies have demonstrated that a red shift occurs in the UV-Vis absorption of the nanoparticles following the addition of an aggregating agent. ${ }^{31,33,74}$ Additionally, this process commonly results in a decrease in the zeta potential of the nanoparticle. ${ }^{33,34}$ A study by Burns et al. examined the relationship between color change and the type of salt, ionic strength, and its associated Debye length. ${ }^{34}$ Their suggestion was that cation adsorption into the stern layer of the nanoparticle played a major role in this process due to a net reduction of nanoparticle surface charge. This process reduced the repulsion between nanoparticles permitting closer interparticle distances and allowing interactions between particles that resulted in color change. Ionic strength and Debye length were not thought to be factors. It is generally postulated that the anion of the aggregating agent has a major effect on SERS enhancement by displacing the reducing agent surrounding the nanoparticle. This process is known as the chloride ion effect. ${ }^{25}$ In previous work in our laboratory we have demonstrated that concentrations as low as $0.5 \mathrm{ng} \mathrm{mL} \mathrm{m}^{-1}$ of benzodiazepines can be detected in urine using supported liquid extraction followed by SERS. ${ }^{45}$ Interestingly, this work also demonstrated that sensitivity 
for the SERS technique varied greatly depending on the nature of the salt used for nanoparticle aggregation, and more particularly on the type of cation as predicted by Burns et al. Thus, the focus of this study was to gain a better understanding of the process of gold nanoparticle aggregation via salt through experiments involving absorption and zeta potential, as well as measuring their effect on SERS enhancements. To perform these experiments, we examined nine different synthetic cannabinoids using a portable Raman spectrometer. These compounds, also known as novel psychoactive substances, provide an interesting test set for optimizing SERS analysis through aggregation, due to the controlled minor variations in structure from one form of the molecule to the next (Figure 13). These changes are typically performed to maintain psychotropic effects yet produce sufficient changes in the molecules to avoid legal scheduling.

It should be noted that there has been a resurgence of interest in recent years on the use of SERS in forensic applications including biological fluids, ${ }^{75}$ trace evidence,,${ }^{76,77}$ gunshot residue ${ }^{75}$ explosives,${ }^{78}$ as well as drug analysis and toxicology ${ }^{45,79-85}$ The various designer drugs that have been examined include JWH-018, JWH-073, JWH-081, JWH-122, 5F-PB-22, $\alpha$-PVP, and AMB-FUBINACA. ${ }^{49,50,86-88}$ The SERS procedure permits rapid analysis at high sensitivity and does not require recognition elements for specificity as does immunoassay based techniques. This can be particularly important in the analysis of novel designer cannabinoids, where structures of illicit substances are constantly changing. These structural variants often lack sufficient cross reactivity with standard immunological testing methods but can be differentiated by SERS techniques. ${ }^{15,89}$ Therefore, an additional and important goal of this paper is to define an 
optimized procedure for analysis of these drugs using colloidal nanoparticles and aggregating agents.
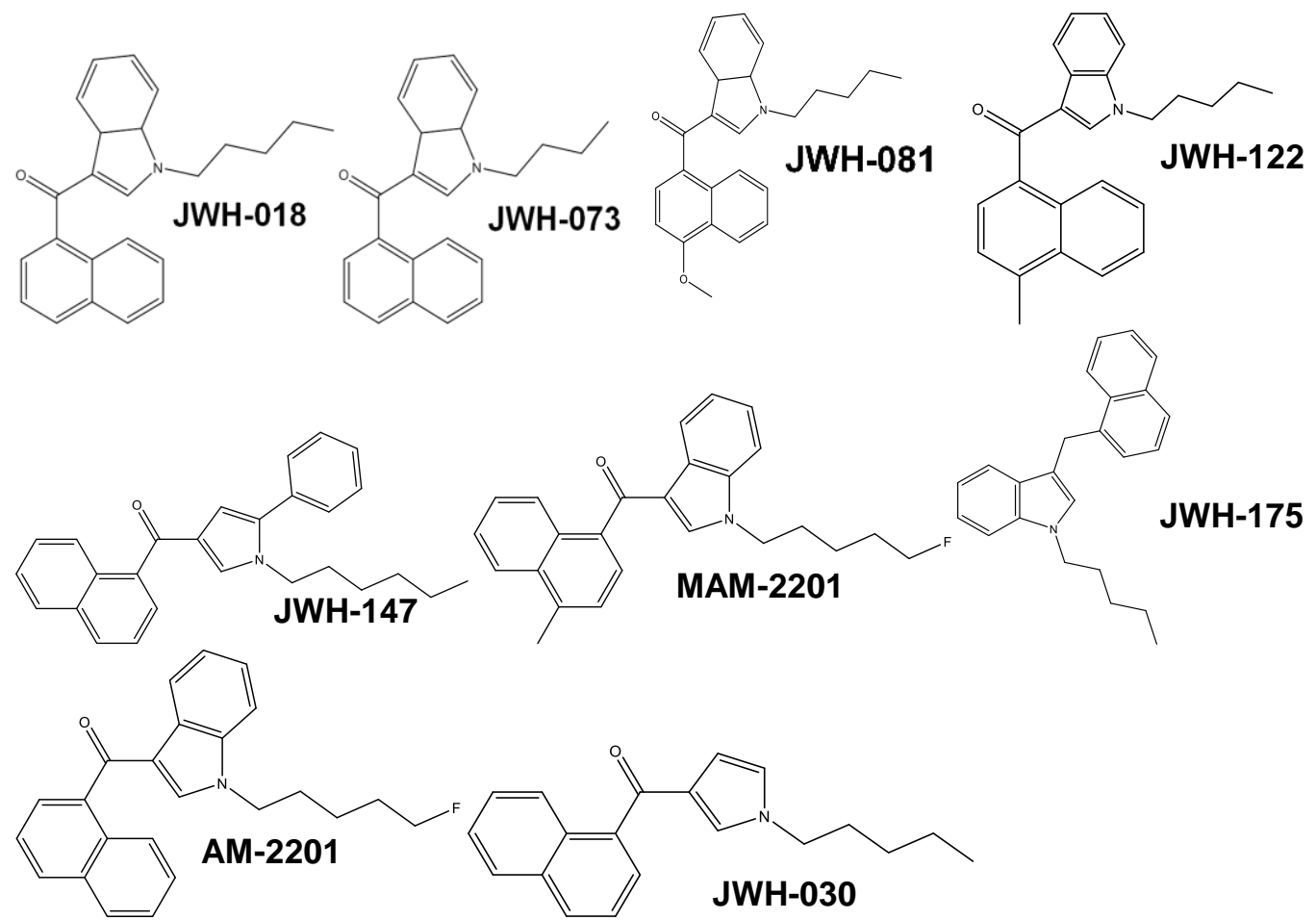

Figure 13: The chemical structures of the nine different synthetic cannabinoids examined in this study.

\subsection{Experimental}

\subsubsection{Materials used}

Preparation of the gold nanoparticle colloids was based on studies previously conducted by Doctor et al., Deriu et al., and Mostowtt et al. ${ }^{44,86,87} 100 \mathrm{~mL}$ gold nanoparticle solutions were prepared reducing tetrachloroauric (iii) acid trihydrate (Acros Organics, LOT\#A0354619) with $4 \mathrm{~mL}$ of a $1 \%$ by weight solution of trisodium citrate dihydrate (Fisher Scientific, LOT \#091236) at pH 5.

Monovalent $\left(\mathrm{K}^{+}\right.$and $\left.\mathrm{Na}^{+}\right)$, divalent $\left(\mathrm{Ca}^{2+}\right.$ and $\left.\mathrm{Mg}^{2+}\right)$, and trivalent $\left(\mathrm{Al}^{3+}\right)$ chlorate, nitrate, and sulfate salts were studied as aggregating agents for the SERS analysis of 
synthetic cannabinoids. The monovalent cations in this study included $\mathrm{KCl}$ (Spectrum), $\mathrm{NaCl}$ (Sigma-Aldrich), $\mathrm{K}_{2} \mathrm{SO}_{4}$ (Fisher Scientific), $\mathrm{Na}_{2} \mathrm{SO}_{4}$ (Spectrum), $\mathrm{NaNO}_{3}$ (Fisher Scientific), and $\mathrm{KNO}_{3}$ (Fisher Scientific). The divalent cations examined include $\mathrm{MgCl}_{2}$ (Fisher Scientific), $\mathrm{CaCl}_{2}$ (Fisher Scientific), $\mathrm{MgSO}_{4}$ (Fisher Scientific), $\mathrm{CaSO}_{4}$ (SigmaAldrich), $\mathrm{Mg}\left(\mathrm{NO}_{3}\right)_{2}$ (Fisher Scientific), and $\mathrm{Ca}\left(\mathrm{NO}_{3}\right)_{2}$ (Fisher Scientific). The trivalent cations included $\mathrm{AlCl}_{3}$, (Fisher Scientific), $\mathrm{Al}_{2}\left(\mathrm{SO}_{4}\right)_{3}$ (Fisher Scientific), and $\mathrm{Al}\left(\mathrm{NO}_{3}\right)_{3}$ (Fisher Scientific).

Synthetic cannabinoid standards (in powder) of JWH-073 (LOT \#1293.1B1.2) and JWH-081 (LOT \#1505.1B1.1) were obtained from Lipomed AG and JWH-030 (LOT \#0443548-16), JWH-147 (LOT \#0444979-9), and MAM-2201 (LOT \#0483939-13) were obtained from Cayman Chemical. DEA exempt preparation of $(\mathrm{mg} \mathrm{mL}-1$ in methanol) JWH-018 (LOT \#0448916-52), JWH-122 (LOT \#0497508-1, JWH-175 (LOT \#045046714), and AM-2201 (LOT \#0499457-1) were obtained from Cayman Chemical. All the above standards were used to create stock standard solutions at concentrations ranging from $25-20000 \mathrm{ng} \mathrm{mL}^{-1}$ in a $10 \%$ methanol $(0.02 \mathrm{M})$ solution.

\subsubsection{UV-vis spectroscopy}

For this procedure $980 \mu \mathrm{L}$ of the gold nanoparticle solution was placed into a quartz Suprasil® cuvette (Hellma Analytics). Then, a select amount of aggregating agent solution was titrated into the cuvette, vortexed, and analyzed via a UV-visible spectrophotometer. The initial stock concentrations for the aggregating agents were as follows: $0.84 \mathrm{M}$ for monovalent cation salts and $0.05 \mathrm{M}$ for divalent and trivalent salts. The amount of aggregating agent solution titrated to the gold nanoparticles varied from $10-100 \mu \mathrm{L}$ (in increments of $10 \mu \mathrm{L}$ ) for monovalent salts and 5-50 $\mu \mathrm{L}$ (in increments of 
$5 \mu \mathrm{L}$ ) for divalent and trivalent salts. The calculated concentration of the aggregating agent in the overall solution was used for data analysis and interpretation of UV-vis results, as well as SERS analysis. All samples were run in triplicate using three nanoparticle solutions that were made on three different days.

\subsubsection{Zetasizer study}

Analysis was conducted on the nanoparticle and aggregating agent interaction via dynamic light scattering at $\mathrm{pH}$ 5. The change in nanoparticle size and zeta potential was measured with three different concentrations for each aggregating agent based upon results obtained from the UV-vis study. The results show that a red shift occurs in the absorbance of the nanoparticles with increasing aggregating agent concentration (Table 4). Three concentrations were determined: the initial aggregating agent concentration titrated in the UV-vis study, the concentration at which a color change of the nanoparticle solution occurred from red to purple, and the concentration at which a color change occurred from purple to grey/clear color. The stock solution concentration of each aggregating agent varied based upon the aggregating agent concentration needed from Table 4. For example, to have a final concentration of $0.048 \mathrm{M} \mathrm{NaCl}$ in the SERS nanoparticle solution, a stock solution of $4.8 \mathrm{M} \mathrm{NaCl}$ was made. The stock concentrations for the aggregating agents ranged from $0.025 \mathrm{M}$ to $4.8 \mathrm{M}$. Not all aggregating agents from the UV-vis study were used in this part of the study due to poor solubility in water. The aggregating agents not examined included: $\mathrm{K}_{2} \mathrm{SO}_{4}, \mathrm{Na}_{2} \mathrm{SO}_{4}, \mathrm{NaNO}_{3}, \mathrm{KNO}_{3}, \mathrm{CaSO}_{4}$, $\mathrm{Mg}\left(\mathrm{NO}_{3}\right)_{2}$, and $\mathrm{Ca}\left(\mathrm{NO}_{3}\right)_{2}$. The aggregating agents examined were $\mathrm{NaCl}, \mathrm{CaCl} 2, \mathrm{MgCl}_{2}$, $\mathrm{AlCl}_{3}, \mathrm{NaNO}_{3}, \mathrm{Ca}\left(\mathrm{NO}_{3}\right)_{2}, \mathrm{Mg}\left(\mathrm{NO}_{3}\right)_{2}, \mathrm{Al}\left(\mathrm{NO}_{3}\right)_{3}, \mathrm{Mg}\left(\mathrm{SO}_{4}\right)_{2}$, and $\mathrm{Al}_{2}\left(\mathrm{SO}_{4}\right)_{3}$. 


\begin{tabular}{|c|c|c|c|}
\hline Aggregating Agent & (A) & (B) & (C) \\
\hline $\mathrm{KCl}$ and $\mathrm{NaCl}$ & $0.0084 \mathrm{M}$ & $0.033 \mathrm{M}$ & $0.048 \mathrm{M}$ \\
\hline $\mathrm{CaCl}_{2}$ and $\mathrm{MgCl}_{2}$ & $0.00025 \mathrm{M}$ & $0.00075 \mathrm{M}$ & $0.0015 \mathrm{M}$ \\
\hline $\mathrm{AlCl}_{3}$ & $0.00025 \mathrm{M}$ & $0.0005 \mathrm{M}$ & $0.0012 \mathrm{M}$ \\
\hline $\mathrm{NaNO}_{3}$ & $0.0084 \mathrm{M}$ & $0.041 \mathrm{M}$ & $0.055 \mathrm{M}$ \\
\hline $\mathrm{Ca}\left(\mathrm{NO}_{3}\right)_{2}, \mathrm{Mg}\left(\mathrm{NO}_{3}\right)$, and $\mathrm{MgSO}_{4}$ & $0.00025 \mathrm{M}$ & $0.001 \mathrm{M}$ & $0.0015 \mathrm{M}$ \\
\hline $\mathrm{Al}\left(\mathrm{NO}_{3}\right)_{3}$ and $\mathrm{Al}_{2}\left(\mathrm{SO}_{4}\right)_{3}$ & $0.00025 \mathrm{M}$ & $0.0005 \mathrm{M}$ & $0.001 \mathrm{M}$ \\
\hline
\end{tabular}

Table 4: (A) Initial concentration of aggregating agents examined, (B) Aggregating agent concentration at which initial color change occurred from red to purple, and (C) Aggregating agent concentration at which initial color change occurred from purple to grey/clear color.

In these experiments, $980 \mu \mathrm{L}$ of the gold nanoparticle solution was placed into a glass vial (Fisher scientific) followed by $10 \mu \mathrm{L}$ of the aggregating agent, vortexed, placed into a polystyrene cuvette and analyzed to determine nanoparticle size. Then, the same solution was transferred to a disposable capillary cell for zeta potential analysis. In a second part of this study $10 \mu \mathrm{L}$ of a $10 \%$ methanol $(0.02 \mathrm{M})$ solution was added to the nanoparticle solution after the addition of the aggregating agent (SERS optimization negative control), vortexed, and analyzed for change in nanoparticle size and zeta potential. This part of the study was conducted to show that the $10 \%$ methanol $(0.02 \mathrm{M})$ solution does not affect the nanoparticle and aggregating agent interaction. All samples were run in triplicate using three nanoparticle solutions made on different days.

The size of the nanoparticles was analyzed using a $4 \mathrm{~mL}$ (10 $\mathrm{mm}$ thickness) polystyrene cuvette (Sarstedt) via dynamic light scattering based upon the theory that particles move by Brownian Motion. The detector was placed at $173^{\circ}$ scattering angle, measuring the scattered light via backscatter detection. The relationship between particle size and Brownian motion can be determined by the Stokes-Einstein equation 


$$
D_{T}=\frac{k_{b} T}{3 \pi \eta d}
$$

where $\mathrm{D}_{\mathrm{T}}$ is the diffusion coefficient, $\eta$ is the viscosity of the fluid, $\mathrm{T}$ is the temperature, $\mathrm{k}_{\mathrm{b}}$ is the Boltzmann constant, and $\mathrm{d}$ is the particle's diameter.30 The instrument measures the correlation of the diffusion of the nanoparticles over time to determine the diffusion coefficient, using the Stokes-Einstein equation to determine the size of the nanoparticles. The rate of decay of the correlation is related to the nanoparticle size. The smaller the nanoparticle, the faster the rate of decay.

The zeta potential of the nanoparticles was determined using a DTS1070 disposable folded capillary cell (Malvern) measuring the electrophoretic mobility of the nanoparticles and converted to zeta potential via the Henry equation

$$
U_{E}=\frac{2 \varepsilon z f(k a)}{3 \eta}
$$

where $U_{E}$ is the electrophoretic mobility, $\eta$ is the viscosity of the fluid, $z$ is the zeta potential, $\varepsilon$ is the dielectric constant, $\mathrm{f}(\mathrm{ka})$ is Henry's function.31 An electric field is applied to the nanoparticle solution, dispersing the particles to the sign opposite of their charge and measures the scattered light at a $13^{\circ}$ from the incident beam. This in combination with the reference beam produces a fluctuation in the signal intensity over time that is proportional to the speed of the nanoparticle and used to determine the electrophoretic mobility.

\subsubsection{SERS optimization}

The results from the UV-vis study determined the aggregating concentration used for each salt in the SERS analysis of the synthetic cannabinoids. SERs analysis was 
performed using the following procedure ${ }^{86}$ First, $980 \mu \mathrm{L}$ of the gold nanoparticle solution was placed in a clear $2 \mathrm{~mL}$ glass vial (Fisher Scientific) followed by $10 \mu \mathrm{L}$ of a select aggregating agent solution and vortexed for 30 seconds. Then, $10 \mu \mathrm{L}$ of the drug stock standard solution was added to the $2 \mathrm{~mL}$ sample vial and vortexed for 30 seconds. The final concentration of each component in the gold nanoparticle solution was as follows: $0.05 \mathrm{mM} \mathrm{HAuCl}_{4}$, between $0.001-0.055 \mathrm{M}$ of aggregating agent, and between 2.5-2000 $\mathrm{ng} \mathrm{mL}^{-1}$ of each synthetic cannabinoid in $0.02 \mathrm{M}$ methanol. Each sample was run in triplicate. A culmination of all the data was used to calculate the limit of detection for each synthetic cannabinoid.

\subsubsection{Instrumentation}

The nanoparticle and aggregating agent interaction was studied using a Varian Cary 100 Bio UV-visible spectrophotometer (absorbance) and Malvern Zetasizer Nano ZS (nanoparticle size and zeta potential) were used in these experiments. The SERS solutions were transferred to a quartz Suprasil@ cuvette (Hellma Analytics) and analyzed using a Thermo Scientific FirstDefenderRM (portable) with a $785 \mathrm{~nm}$ laser at high power $(250 \mathrm{~mW})$ with a resolution of 7 to $10.5 \mathrm{~cm}^{-1}$ (FWHM).

\subsection{Results and discussion}

In this study, nine different synthetic cannabinoids (JWH-018, JWH-030, JWH073, JWH-081, JWH-122, JWH-147, JWH-175, AM-2201, and MAM-2201 were examined in order to gain a better understanding of the nanoparticle, aggregating agent, and drug interaction to produce the achieved SERS enhancement as well as optimization of the detection of synthetic cannabinoids. This study is divided into two parts. The first part examines how different aggregating agents within varying concentrations affect the 
gold nanoparticle destabilization. The second part evaluates the effect of monovalent, divalent, and trivalent chloride, sulfate, and nitrate salts to determine the optimal aggregating agent concentration to detect the nine synthetic cannabinoids examined. The objective of this study is to explain why certain salts work better than others for the detection of synthetic cannabinoids in solution.

The gold nanoparticles were created via a citrate reduction method, similar to Doctor et al. ${ }^{44}$ The UV-VIS peak absorbance was $521 \pm 0.7 \mathrm{~nm}$. The peak absorption and size distribution were similar to previous results obtained by Mostowtt et al. and Doctor et al. ${ }^{44,86}$

\subsubsection{Aggregating agent optimization}

In the first part of this study, 15 different aggregating agents were examined including monovalent, divalent, and trivalent chloride, sulfate, and nitrate salts. The goal of this part of the study was to determine the concentration at which nanoparticle color change and aggregation (a change from red to grey/clear solution color) occurred for each salt, while gaining a better understanding of the mechanism by which the cation and anion of the aggregating agent interact with the nanoparticles to produce SERS enhancement.

Kim et al. examined the effect of gold nanoparticle aggregation following the addition of benzyl mercaptan as the aggregating agent over a period of 2 hours. They found that as time passed the UV-vis spectrum of the nanoparticles red shifted and decreased the original plasmon band at $520 \mathrm{~nm}$, and a new plasmon band at approximately $720 \mathrm{~nm}$ appeared. ${ }^{33}$ In addition, Kim et al. studied the change in the zeta potential with the addition of benzyl mercaptan. The results showed a decrease in the zeta 
potential of the gold nanoparticles after the addition of benzyl mercaptan. Two hours following the addition of the benzyl mercaptan, the zeta potential continued to decrease. 8 The results of the UV-vis and Zetasizer study indicate that as an aggregating agent is added to the nanoparticle solution the nanoparticles destabilize and come closer together resulting in a decrease in the zeta potential.

In a study by Bell et al., the UV spectra of gold colloidal nanoparticles of various sizes following the addition of $\mathrm{MgSO}_{4}$ was examined to determine an optimal SERS method to detect thiophenol in solution. ${ }^{74}$ For the $46 \mathrm{~nm}$ gold colloids, as the amount of $\mathrm{MgSO}_{4}$ increased from $0.00055 \mathrm{~mol} \mathrm{dm}^{-3}$ to $0.011 \mathrm{~mol} \mathrm{dm}^{-3}$ the absorbance of the original plasmon band decreased and an additional band appeared at $640 \mathrm{~nm}$ with shifts to $800 \mathrm{~nm}$ at higher $\mathrm{MgSO}_{4}$ concentrations. For the $30 \mathrm{~nm}$ gold colloids, as $0.0033 \mathrm{~mol}$ $\mathrm{dm}^{-3} \mathrm{MgSO}_{4}$ was added to the colloid solution, an additional band at $800 \mathrm{~nm}$ appeared. As the $\mathrm{MgSO}_{4}$ concentration increased from $0.0033 \mathrm{~mol} \mathrm{dm}^{-3}$ to $0.024 \mathrm{~mol} \mathrm{dm}^{-3}$, the absorbance of the original plasmon band and the new plasmon band decreased. Bell et al. considered this reaching a 'fully aggregated' plateau. ${ }^{74}$ The authors attributed the effect of the $\mathrm{MgSO}_{4}$ on the gold colloid solution to the formation of gold colloid aggregates. In this study, the aggregating agent was titrated in varying ratios with the gold nanoparticle solution. The concentration at which any color change occurred was noted (Table 1) and analyzed via UV-visible spectroscopy to access the change in absorbance of the nanoparticle solution as the salt concentration increases (Figure 14). The concentration of the aggregating agent at the color transition was calculated based upon the amount of salt added to the nanoparticle solution. The results obtained in this study agreed with the previous work mentioned above. For all the salts examined, as the 
concentration increased, the absorbance at $521 \mathrm{~nm}$ decreased and shifted to a higher wavelength. In addition, as the salt concentrations increased above the values noted in Table 1 Row C, a decrease in the absorbance of the original and red shifted plasmon band was seen. A 'fully aggregated' plateau was achieved. Therefore, at concentrations above Table 1 Row C for each salt, SERS enhancement may be less effective for the gold nanoparticle solutions formed in this study.

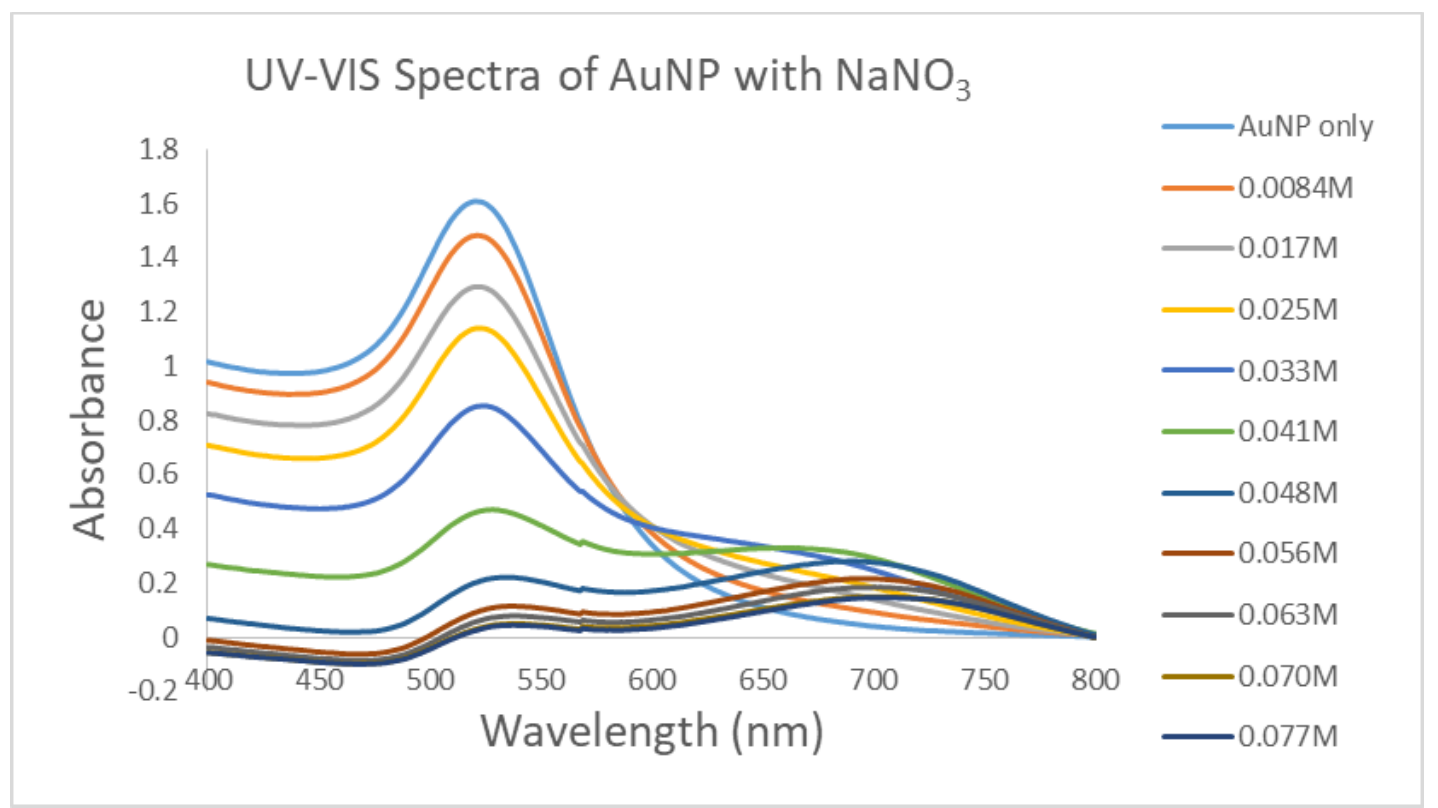

Figure 14: Characterization of the changes in absorption of the gold nanoparticle solution as concentration of $\mathrm{NaNO}_{3}$ increases.

Using the data collected, the change in maximum absorbance at $521 \mathrm{~nm}$ was plotted versus the change in concentration. When comparing monovalent, divalent, and trivalent cations, a higher concentration of the monovalent cations was needed to achieve color change compared to the divalent and trivalent cations (Figure 15). However, when comparing the anions of each monovalent, divalent, and trivalent cation, no major difference in the red shift of the surface plasmon band was observed (Figure 16). These 
results demonstrate the importance of the cation in the color change and aggregation process.
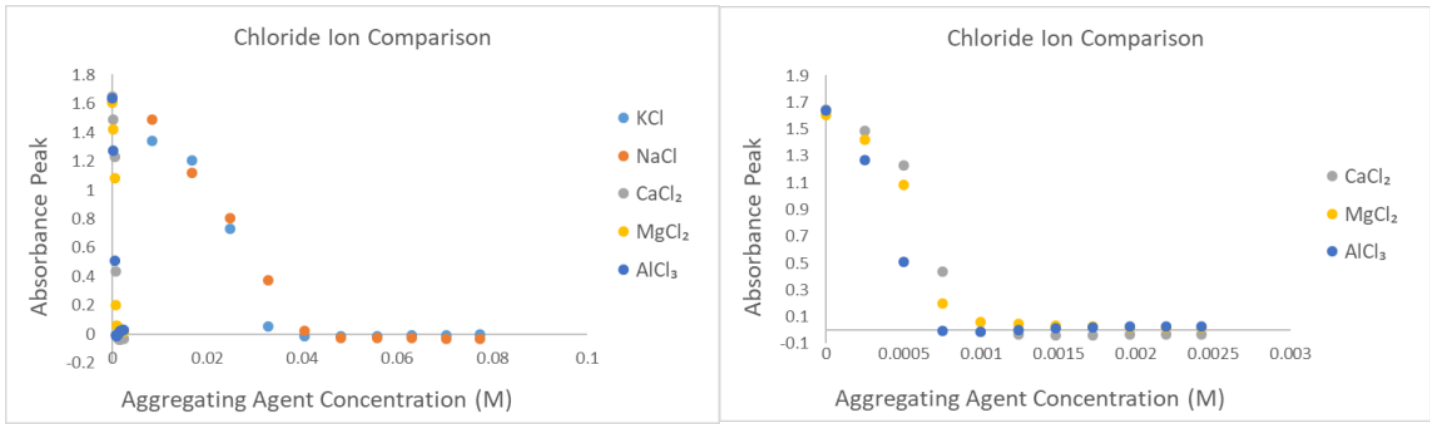

Figure 15: Comparing changes in absorption of the gold nanoparticle solution as concentration of various chloride salts increase.
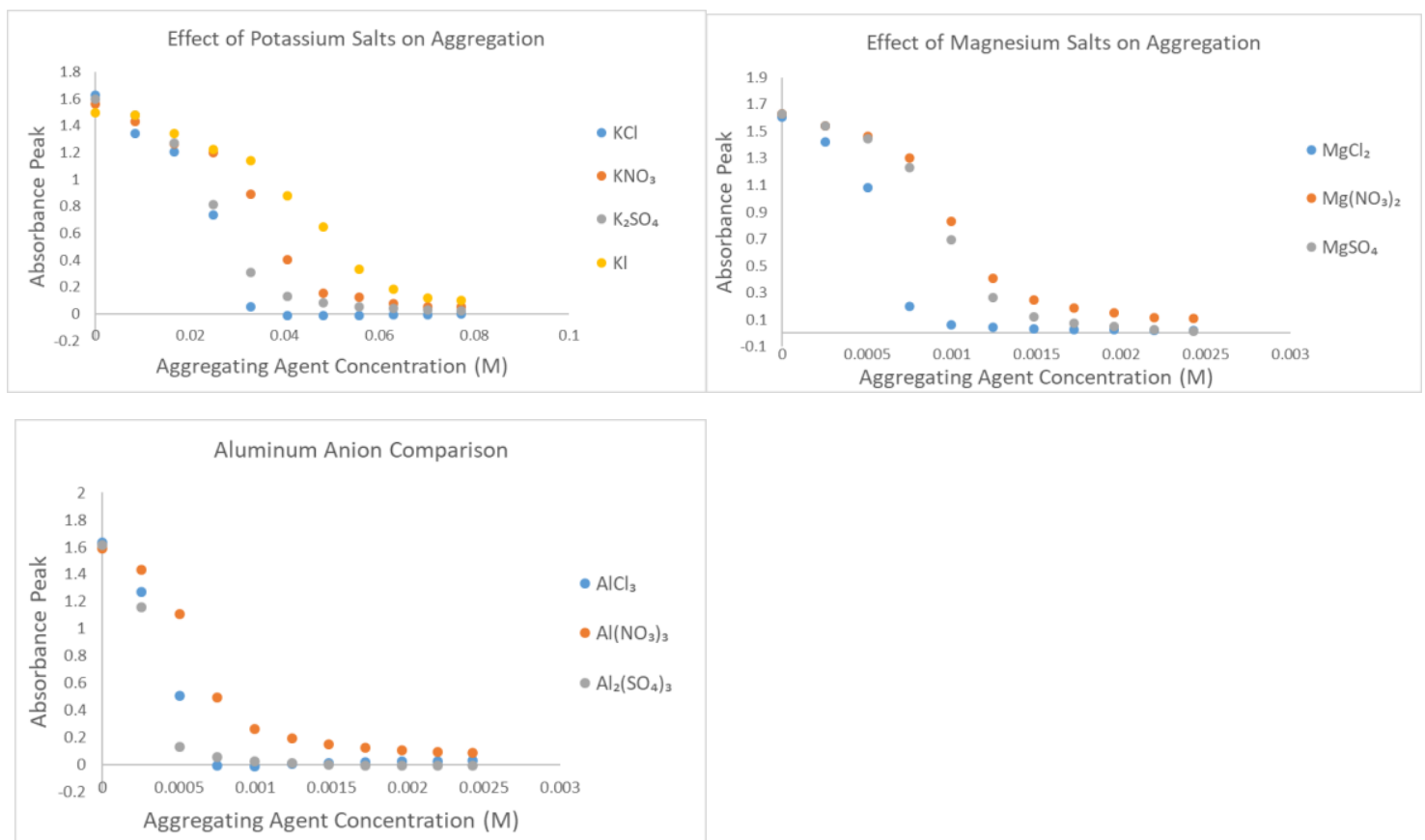

Figure 16: Characterization of the changes in absorption of the nanoparticles as concentration of the cation increases.

Burns et al. investigated the effect of ionic strength on gold nanoparticle aggregation and how this relates to the visible color change seen, from red to purple, after the addition of various aggregating agents. In their study, the ionic strength, activity coefficient and Debye length (particle-to-particle mean distance) were calculated. ${ }^{34}$ From 
their calculations, the higher the ionic strength (i.e. the higher the salt concentration), the lower the Debye length between nanoparticles, resulting in a stronger surface plasmon interaction between nanoparticles. ${ }^{34}$ The divalent cations examined produced a color change at a lower concentration than the monovalent cations and thus had a larger calculated Debye length. Therefore, the color change seen after the addition of the aggregating agent may not be related to the calculated Debye length between nanoparticles, but instead the result of the cation adsorption to the surface of the nanoparticles which permits closer association. ${ }^{34}$ However, the authors did not ex 4 mine the effect of higher concentrations of salts on aggregation.

In general, the work of Burns et al. indicated that the cation of the aggregating agent influences nanoparticle destabilization. Burns et al. showed a change in the zeta potential from negative to positive between the beginning and the titration end point of the aggregating agent. ${ }^{34}$ Their results show that the destabilization of the nanoparticles through the use of an aggregating agent does not just displace the negatively charged reducing agent, in this case citrate, with the anion of the aggregating agent salt. It also shows that the cation assists in the destabilization of the nanoparticles. In our results, we examined a wider range of salt concentrations and showed that divalent cations also produce aggregation at a lower salt concentration than the monovalent cations (Table 4 and Figure 15).

The presence of aggregation can be elucidated by studying the change in nanoparticle size and zeta potential after the addition of aggregating agents. As the aggregating agent was added to the nanoparticles, the distribution of the average nanoparticle size changed (Table 5). The addition of the aggregating agent destabilizes 
the nanoparticle, reducing the electrostatic attraction between particles bringing them closer together and producing aggregates. ${ }^{92,93}$ It is important to note that the Zetasizer interprets aggregated nanoparticles as one larger nanoparticle. Due to this, the calculated increases in average nanoparticle sizes indicate agglomeration. Thus, the sizes produced indicate the effect of nanoparticle destabilization is not just closer interactions between particles but the formation of aggregates (Figure 17).

\section{AuNP Only}

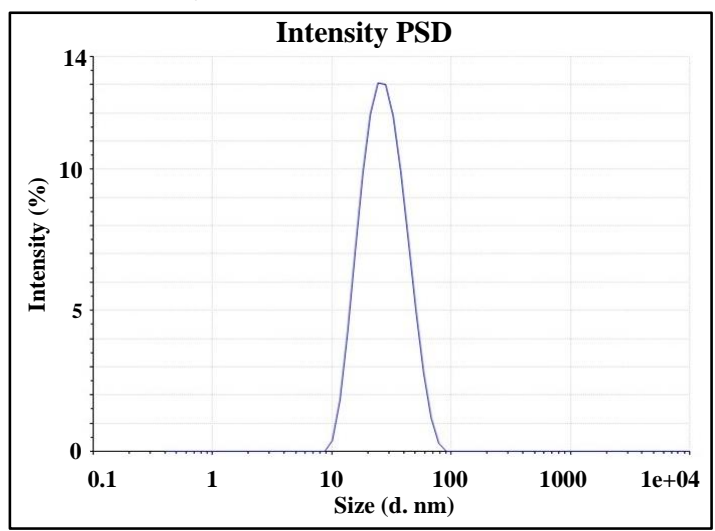

\subsection{NaNO}

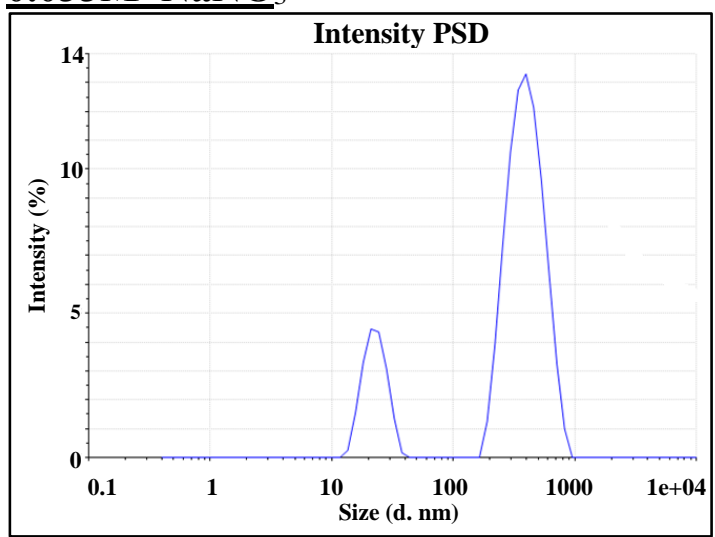

\section{$0.0084 \mathrm{M} \mathrm{NaNO}_{3}$}

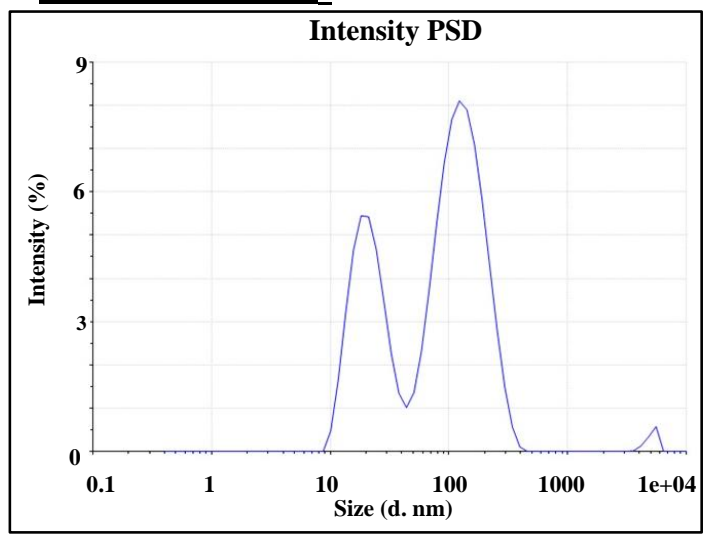

\section{$0.048 \mathrm{M} \mathrm{NaNO}$}

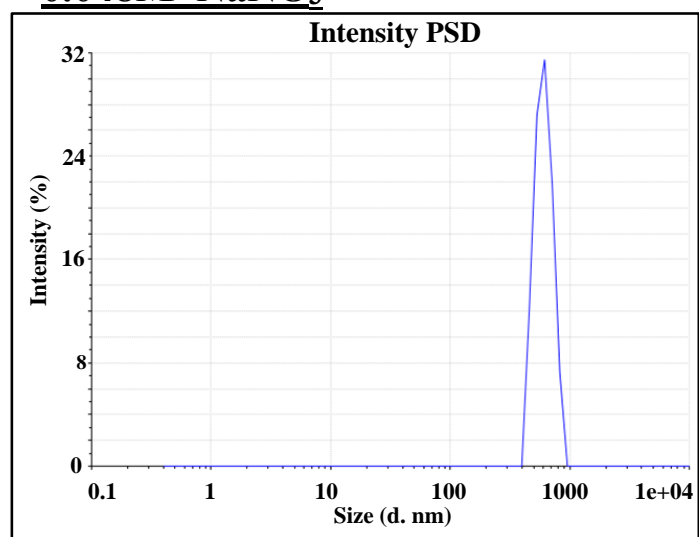

Figure 17: Characterization of the change in size (diameter) of the gold nanoparticles as concentration of $\mathrm{NaNO}_{3}$ increases. 


\begin{tabular}{|c|c|c|c|c|c|}
\hline $\begin{array}{l}\text { Salt } \\
\text { Concentration }\end{array}$ & $\begin{array}{l}\text { AuNP } \\
\text { Size Avg } \\
(\mathrm{nm})\end{array}$ & $\begin{array}{l}\text { AuNP } \\
\text { Size Std } \\
\text { Dev (nm) }\end{array}$ & $\begin{array}{l}\text { Salt } \\
\text { Concentration }\end{array}$ & $\begin{array}{c}\text { AuNP } \\
\text { Size Avg } \\
\text { (nm) }\end{array}$ & $\begin{array}{c}\text { AuNP } \\
\text { Size Std } \\
\text { Dev (nm) }\end{array}$ \\
\hline None & 27.7 & 6.7 & $0.0015 M$ & 181 & 50 \\
\hline $0.0084 \mathrm{M} \mathrm{NaCl}$ & 51.9 & 14 & $\mathrm{Ca}\left(\mathrm{NO}_{3}\right)_{2}$ & & \\
\hline $0.033 \mathrm{M} \mathrm{NaCl}$ & 130 & 51 & $0.00025 \mathrm{M}$ & 51.0 & 8.7 \\
\hline $0.048 \mathrm{M} \mathrm{NaCl}$ & 130 & 18 & $\mathrm{Mg}\left(\mathrm{NO}_{3}\right)_{2}$ & & \\
\hline $0.00025 \mathrm{M}$ & 41.5 & 4.3 & $0.001 \mathrm{M}$ & 126 & 29 \\
\hline $\mathrm{CaCl}_{2}$ & & & $\mathrm{Mg}\left(\mathrm{NO}_{3}\right)_{2}$ & & \\
\hline $\begin{array}{l}0.00075 \mathrm{M} \\
\mathrm{CaCl}\end{array}$ & 132 & 48 & $\begin{array}{l}0.0015 \mathrm{M} \\
\mathrm{Mg}\left(\mathrm{NO}_{3}\right)_{2}\end{array}$ & 154 & 54 \\
\hline $0.001 \mathrm{M} \mathrm{CaCl}_{2}$ & 138 & 18 & $0.00025 \mathrm{M}$ & 45.5 & 3.9 \\
\hline $\begin{array}{l}0.00025 \mathrm{M} \\
\mathrm{MgCl}_{2}\end{array}$ & 31.1 & 5.8 & $\begin{array}{l}\mathrm{Al}\left(\mathrm{NO}_{3}\right)_{3} \\
0.0005 \mathrm{M}\end{array}$ & 33.9 & 7.6 \\
\hline $\begin{array}{l}0.00075 \mathrm{MI} \\
\mathrm{MgCl}_{2}\end{array}$ & 87.9 & 37 & $\begin{array}{l}\mathrm{Al}\left(\mathrm{NO}_{3}\right)_{3} \\
0.001 \mathrm{M}\end{array}$ & 186 & 31 \\
\hline $\begin{array}{l}0.0015 \mathrm{MI} \\
\mathrm{MgCl}_{2}\end{array}$ & 164 & 44 & $\begin{array}{l}\mathrm{Al}\left(\mathrm{NO}_{3}\right)_{3} \\
0.00025 \mathrm{M}\end{array}$ & 45.1 & 7.9 \\
\hline $\begin{array}{l}0.00025 \mathrm{M} \\
\mathrm{AlCl}_{3}\end{array}$ & 56.1 & 12 & $\begin{array}{l}\mathrm{MgSO}_{4} \\
0.001 \mathrm{M} \\
\mathrm{MgSO}\end{array}$ & 148 & 35 \\
\hline $0.0005 \mathrm{M} \mathrm{AlCl}_{3}$ & 46.5 & 11 & $0.0015 M$ & 209 & 77 \\
\hline $0.0012 \mathrm{M} \mathrm{AlCl}_{3}$ & 129 & 24 & $\mathrm{MgSO}_{4}$ & & \\
\hline $\begin{array}{l}\mathbf{0 . 0 0 8 4 M} \\
\mathrm{NaNO}_{3}\end{array}$ & 44.0 & 9.8 & $\begin{array}{l}0.00025 \mathrm{M} \\
\mathrm{Al}_{2}\left(\mathrm{SO}_{4}\right)_{3}\end{array}$ & 35.8 & 12 \\
\hline $0.041 \mathrm{M} \mathrm{NaNO}_{3}$ & 188 & 31 & $0.0005 \mathrm{M}$ & 143 & 39 \\
\hline $\begin{array}{l}0.055 \mathrm{M} \mathrm{NaNO}_{3} \\
0.001 \mathrm{M} \\
\mathrm{Ca}\left(\mathrm{NO}_{3}\right)\end{array}$ & 198 & 53 & $\begin{array}{l}\mathrm{Al}_{2}\left(\mathrm{SO}_{4}\right)_{3} \\
\mathrm{O.001 \textrm {M }} \\
\mathrm{Al}_{2}\left(\mathrm{SO}_{4}\right)_{3}\end{array}$ & 175 & 27 \\
\hline
\end{tabular}

Table 5: Characterization of the change in the average size (diameter) of the gold nanoparticles as the salt concentration increases.

The effect of the addition of the aggregating agent is demonstrated in Figure 14,

which shows that as the aggregating agent was added to the nanoparticle solution, a shift in the average size of the particles occurs. As the initial amount of salt is added to the nanoparticles, the single nanoparticle size distribution peak splits into two peaks 
demonstrating the start of the aggregation process; however, at this stage no visible color change has occurred. As the salt concentration increases to cause a color change from red to purple, an increase in the number of destabilized nanoparticles is seen. Upon complete aggregation, the nanoparticle solution turns to a grey/clear color and a single peak is formed. This data demonstrates that the addition of aggregating agent not only destabilizes the nanoparticles and alter the spectra, but it clearly induces the formation aggregates, even at the earliest stages.

Another factor to consider is the effect of the aggregating agent on the zeta potential of the nanoparticle (Table 6). For these nanoparticles, citrate stabilizes the gold colloids and results in an overall negative zeta potential. In this study, the zeta potential decreased as the salt concentration increased, indicating a reduction in charge, leading to closer interactions between particles and thus aggregation. This result is consistent with the nanoparticle size study. In general, nanoparticles are considered stable if the nanoparticle solution has an average zeta potential value equal to or greater than the average nanoparticle size. Destabilization occurs when the zeta potential is less than the average nanoparticle size. In this study, the nanoparticle solution without the addition of any aggregating agent had an average nanoparticle size of $27.7 \pm 6.7 \mathrm{~nm}$ and an average zeta potential of $-35.9 \pm 2.1 \mathrm{mV}$. When the recorded zeta potential $(35.9 \mathrm{mV})$ is higher than the size $(27.7 \mathrm{~nm})$, the nanoparticles in solution are considered stable. Comparing the results following the addition of the aggregating agents at all the concentrations examined, the nanoparticle size value was greater than that of the zeta potential and aggregation occurred as shown in Figure 17. 


\begin{tabular}{|c|c|c|c|c|}
\hline & \multicolumn{2}{|c|}{ No MeOH Added } & \multicolumn{2}{|l|}{ MeOH Added } \\
\hline $\begin{array}{l}\text { Salt and } \\
\text { Concentration }\end{array}$ & $\begin{array}{l}\text { Zeta Potential } \\
\text { Average }(\mathrm{mV})\end{array}$ & $\begin{array}{l}\text { Zeta Potential } \\
\text { Standard Dev } \\
(\mathrm{mV})\end{array}$ & $\begin{array}{l}\text { Zeta Potential } \\
\text { Average }(\mathrm{mV})\end{array}$ & $\begin{array}{l}\text { Zeta Potential } \\
\text { Standard Dev }(\mathrm{mV})\end{array}$ \\
\hline None & -35.9 & 2.1 & -35.1 & 3.8 \\
\hline $0.0084 \mathrm{M} \mathrm{NaCl}$ & -34.8 & 5.8 & -38.6 & 2.6 \\
\hline 0.033M NaCl & -46.4 & 4.9 & -52.9 & 6.2 \\
\hline 0.048M NaCl & -30.0 & 7.2 & -34.1 & 3.1 \\
\hline $0.00025 \mathrm{M} \mathrm{CaCl}_{2}$ & -30.5 & 1.8 & -31.9 & 1.8 \\
\hline $0.00075 \mathrm{M} \mathrm{CaCl}_{2}$ & -25.6 & 1.3 & -24.3 & 0.8 \\
\hline $0.001 \mathrm{M} \mathrm{CaCl}_{2}$ & -18.7 & 0.7 & -21.0 & 1.7 \\
\hline $0.00025 \mathrm{M} \mathrm{MgCl}_{2}$ & -34.6 & 2.2 & -30.8 & 1.5 \\
\hline $0.00075 \mathrm{M} \mathrm{MgCl}_{2}$ & -26.7 & 1.1 & -27.1 & 0.9 \\
\hline $0.0015 \mathrm{M} \mathrm{MgCl}_{2}$ & -22.3 & 1.0 & -21.7 & 1.0 \\
\hline $0.00025 \mathrm{M} \mathrm{AlCl}_{3}$ & -36.2 & 4.3 & -36.5 & 3.2 \\
\hline $0.0005 \mathrm{M} \mathrm{AlCl}_{3}$ & -32.7 & 4.1 & -31.3 & 3.2 \\
\hline $0.0012 \mathrm{M} \mathrm{AlCl}_{3}$ & -18.3 & 3.3 & -19.6 & 5.2 \\
\hline $\mathrm{o.0084}_{\mathrm{M} \mathrm{NaNO}}$ & -34.5 & 5.7 & -38.8 & 1.4 \\
\hline $\mathrm{o.041M} \mathrm{NaNO}_{3}$ & -26.7 & 8.0 & -36.9 & 5.3 \\
\hline $\mathrm{0.055M} \mathrm{NaNO}_{3}$ & -27.1 & 10 & -31.1 & 1.9 \\
\hline $0.00025 \mathrm{M} \mathrm{Ca}\left(\mathrm{NO}_{3}\right)_{2}$ & -29.3 & 1.3 & -31.7 & 1.8 \\
\hline $0.00075 \mathrm{M} \mathrm{Ca}\left(\mathrm{NO}_{3}\right)_{2}$ & -19.0 & 3.9 & -21.1 & 1.2 \\
\hline $0.0015 \mathrm{M} \mathrm{Ca}\left(\mathrm{NO}_{3}\right)_{2}$ & -16.1 & 4.3 & -13.6 & 1.2 \\
\hline $0.00025 \mathrm{M} \mathrm{Mg}\left(\mathrm{NO}_{3}\right)_{2}$ & -28.9 & 1.4 & -31.7 & 1.7 \\
\hline $0.001 \mathrm{M} \mathrm{Mg}\left(\mathrm{NO}_{3}\right)_{2}$ & -21.8 & 1.5 & -21.5 & 0.5 \\
\hline $0.0015 \mathrm{M} \mathrm{Mg}\left(\mathrm{NO}_{3}\right)_{2}$ & -17.8 & 1.4 & -19.2 & 0.8 \\
\hline $0.00025 \mathrm{M} \mathrm{Al}\left(\mathrm{NO}_{3}\right)_{3}$ & -33.3 & 3.2 & -41.1 & 1.2 \\
\hline $0.0005 \mathrm{M} \mathrm{Al}\left(\mathrm{NO}_{3}\right)_{3}$ & -27.7 & 7.7 & -35.2 & 1.7 \\
\hline $0.001 \mathrm{M} \mathrm{Al}\left(\mathrm{NO}_{3}\right)_{3}$ & -12.4 & 4.3 & -12.5 & 3.0 \\
\hline $0.00025 \mathrm{M} \mathrm{MgSO}_{4}$ & -29.5 & 2.6 & -32.6 & 1.2 \\
\hline $\mathrm{0.001M} \mathrm{MgSO}_{4}$ & -21.7 & 1.1 & -23.5 & 1.0 \\
\hline $\mathrm{0.0015M} \mathrm{MgSO}_{4}$ & -13.7 & 1.9 & -15.2 & 5.8 \\
\hline $0.00025 \mathrm{M} \mathrm{Al}_{2}\left(\mathrm{SO}_{4}\right)_{3}$ & -32.3 & 3.7 & -39.7 & 2.0 \\
\hline $0.0005 \mathrm{M} \mathrm{Al}_{2}\left(\mathrm{SO}_{4}\right)_{3}$ & -16.9 & 3.2 & -17.7 & 2.3 \\
\hline $0.001 M \mathrm{Al}_{2}\left(\mathrm{SO}_{4}\right)_{3}$ & -6.9 & 6.3 & -2.6 & 0.4 \\
\hline
\end{tabular}

Table 6: A comparison of the changes in the gold nanoparticle solution zeta potential after the addition of chloride, sulfate and nitrate salts with and without the addition of 10 $\mu \mathrm{L}$ of $10 \%$ methanol $(0.02 \mathrm{M})$ solution. 
To determine if the addition of methanol has any effect on the nanoparticle and salt destabilization, a comparison of these results was performed with and without the addition of $10 \mu \mathrm{L}$ of $10 \%$ methanol $(0.02 \mathrm{M})$. In our analytical method the synthetic cannabinoids were dissolved and/or diluted in a $10 \%$ methanol $(0.02 \mathrm{M})$ solution. The results seen in Table 3, demonstrate that the addition of the $10 \%$ methanol $(0.02 \mathrm{M})$ solution had no effect on zeta potential.

A few studies investigating the effect of salts on aggregation reference the Hofmeister series and how the values produced relate to the critical coagulation concentration (CCC). ${ }^{35,36,94-98}$ The Hofmeister series organizes cations and anions according to their ability to salt-out (kosmotropic) or salt-in (chaotropic) proteins. The $\mathrm{CCC}$ is the concentration of the aggregating agent counterions needed to induce destabilization of the nanoparticles at a demarcation between fast and slow aggregation. ${ }^{35,36}$ A recent study by Tian et al. determined the CCC of nitrate salt aggregating agents containing various alkali cations for montmorillonite nanoparticles (negatively charged nanoparticles). They determined that larger alkali cations need a lower concentration to reach the CCC compared to smaller alkali cations. ${ }^{96}$ Oncsik et al. studied various salts of monovalent cations and anions of the Hofmeister series and their effect on the CCC of polystyrene latex nanoparticles functionalized with sulfate (negatively charged stern layer) or amidine (positively charged stern layer). For the positively and negatively charged polystyrene latex nanoparticles, the more hydrated cations and anions needed a higher concentration to reach the CCC. ${ }^{35}$ Therefore, a higher concentration of a strongly hydrated cation or anion should be needed to produce the same end effect of nanoparticle destabilization. 
Pavlovic et al. studied the effects of various potassium monovalent, divalent, trivalent, and tetravalent anions salts on of the aggregation of $\mathrm{MgAl}-\mathrm{NO}_{3}-\mathrm{LDH}$ particles. The monovalent anions followed the indirect Hofmeister series, except for $\mathrm{HCO}^{3-}$, which achieved the $\mathrm{CCC}$ at the lowest concentration. In addition, the divalent, trivalent, and tetravalent anions salts achieved the CCC at a lower concentration than the monovalent anions. However, as the concentration of $\mathrm{PO}_{4}{ }^{3-}, \mathrm{Fe}(\mathrm{CN})_{6}{ }^{3-}$, and $\mathrm{Fe}(\mathrm{CN})_{6}{ }^{4-}$ increased, a stabilization of the nanoparticles occurred, creating a second and third CCC, which could be due to hydrophobic interactions, hydrogen bonding, or electrostatic interactions. ${ }^{37}$ Rouster et al. determined the critical coagulation ionic strength (CCIS) of similar potassium salts on positively and negatively charged titania nanosheets. The CCIS relates the ionic strength of the salt to the CCC. The charge of the titania nanosheets was created by changing the $\mathrm{pH}$. The result of the positively charged titania nanosheets agreed with the results obtained by Pavlovic et al. The higher the valence of the anion, the lower the concentration needed to reach the CCIS following the Schulze-Hardy rule. However, the negatively charged titania nanosheets, followed the inverse the Schulze-Hardy rule. ${ }^{97}$

Our research study included an examination of monovalent and divalent anions as well as monovalent, divalent, and trivalent cations. When comparing the chloride, sulfate, and nitrate anions, the results showed a similar trend on the effect on nanoparticle size and zeta potential (Tables 5 and 6). The cations examined in our study can be ranked from strongly to weakly hydrated as follows: $\mathrm{Al}^{3+}>\mathrm{Mg}^{2+}>\mathrm{Ca}^{2+}>\mathrm{Na}^{+}$. Yet, the cation that produced the lowest $\mathrm{CCC}$ was $\mathrm{Al}^{3+}$ and the cation that produced the highest was $\mathrm{Na}^{+}$.

These results indicate that the destabilization produced by these cationic salts follow an indirect Hofmeister series. ${ }^{96,97}$ Salts which keep negatively charged proteins 
stable also produce lower CCCs. These results are in accordance with the Derjaguin, Landau, Verwey, and Overbeek (DLVO) theory which states that colloids in solution stabilize due to van der Waals dispersion forces and Coulomb repulsions (electrostatic interactions). ${ }^{25,98}$ In addition, the Schulze-Hardy rule states that the destabilization of the nanoparticles is affected by the valence of the aggregating agent cation. A higher valence will induce the $\mathrm{CCC}$ at a lower concentration. 40 The results in our study agree with the Schulze-Hardy rule. When comparing the various chloride salts examined in our study, the higher charged cation, $\mathrm{Al}^{3+}$, reached the $\mathrm{CCC}$ at a lower salt concentration. In addition, the study on the change in nanoparticle size and zeta potential demonstrated that the type of cation used as the aggregating agent can affect the $\mathrm{CCC}$ of gold nanoparticles. This is important to understand SERS enhancement. In the final portion of this study, the ability to detect nine different synthetic cannabinoids via SERS was examined to determine an optimal aggregating agent to use for further studies on biological samples.

\subsubsection{SERS optimization}

The aggregating agent concentrations used in this part of the study were the concentrations indicated in Table 4, Row C. Initially, the SERS spectrum of the negative controls were examined. The negative controls only contained the gold nanoparticles, an aggregating agent, and 0.02 $\mathrm{M}$ of methanol (Figure 18). The SERS spectrum produced resulted mainly from the background signal of the aggregating agents and varied with the type of salt used. 


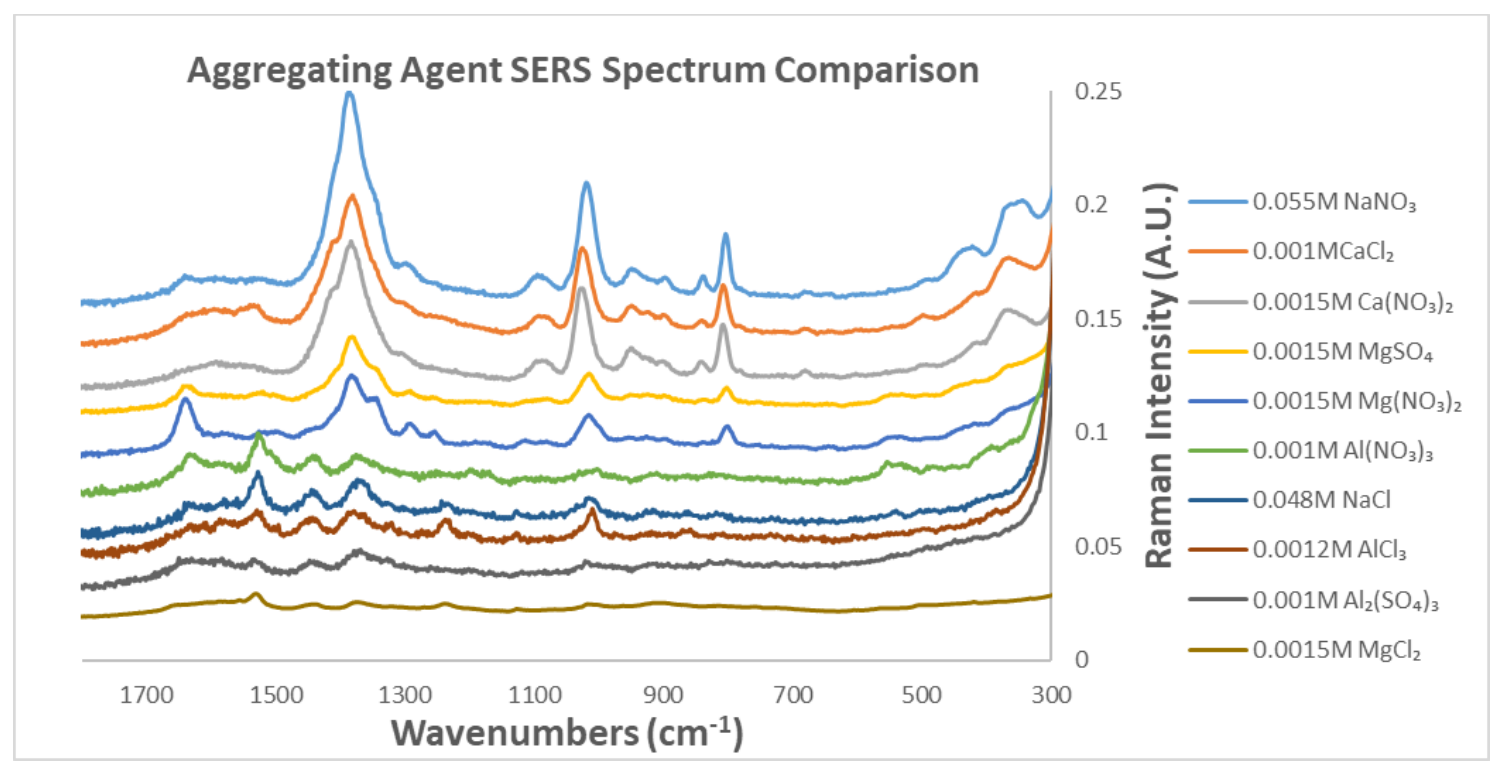

Figure 18: A comparison of the SERS spectrum of ten different aggregating agents (negative controls). The negative controls contained gold nanoparticles, various aggregating agents at the concentrations noted in Table 1 Row $\mathrm{C}$, and $0.02 \mathrm{M}$ methanol.

Experiments were also performed examining the effect of the different salts on the spectra of synthetic cannabinoids (Figure 19 A and B). When using the sulfate, nitrate, $\mathrm{CaCl}_{2}$, and $\mathrm{NaCl}$ as aggregating agents, the strength of signal intensity of the negative control spectrum interfered with the weak spectrum of the analyte and could not be detected using the portable Raman system. Best results were obtained using $\mathrm{MgCl}_{2}$ and $\mathrm{AlCl}_{3}$. Based upon the spectra produced, the limits of detection (LOD) were determined using the peaks with the highest intensity (Table 7). The salt that produced the lowest limit of detection for all compounds examined was $\mathrm{MgCl}_{2}$. The SERS spectrum of each compound using $\mathrm{MgCl}_{2}$ as the aggregating agent can be seen in Figure 20. 


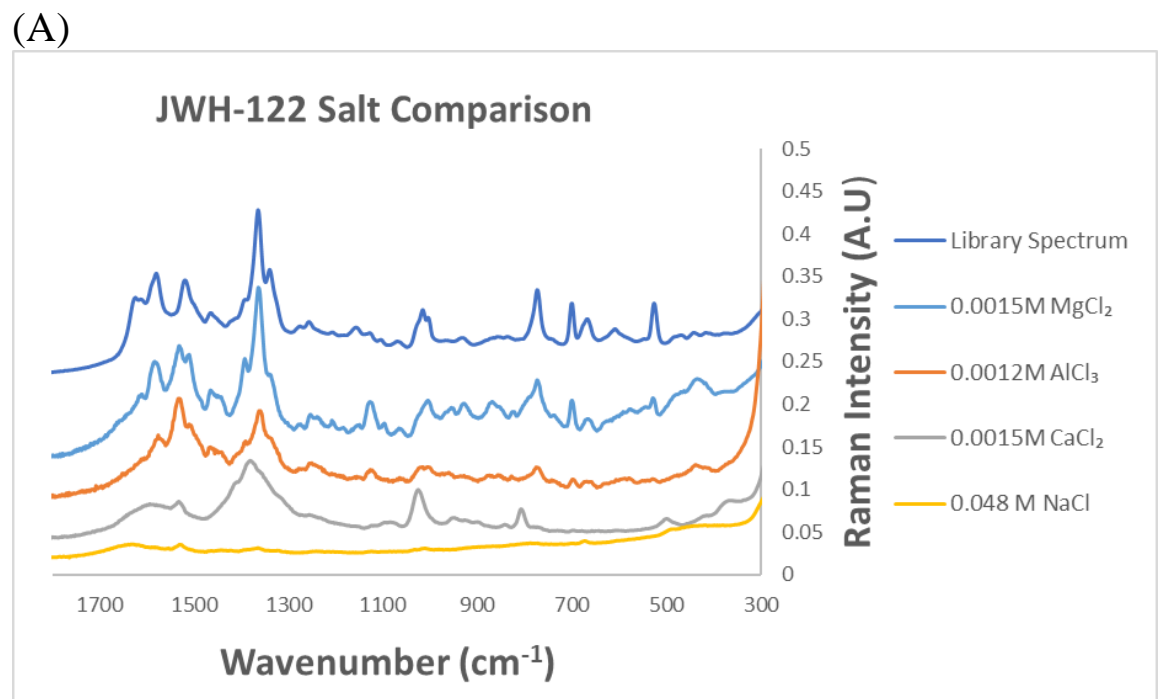

(B)

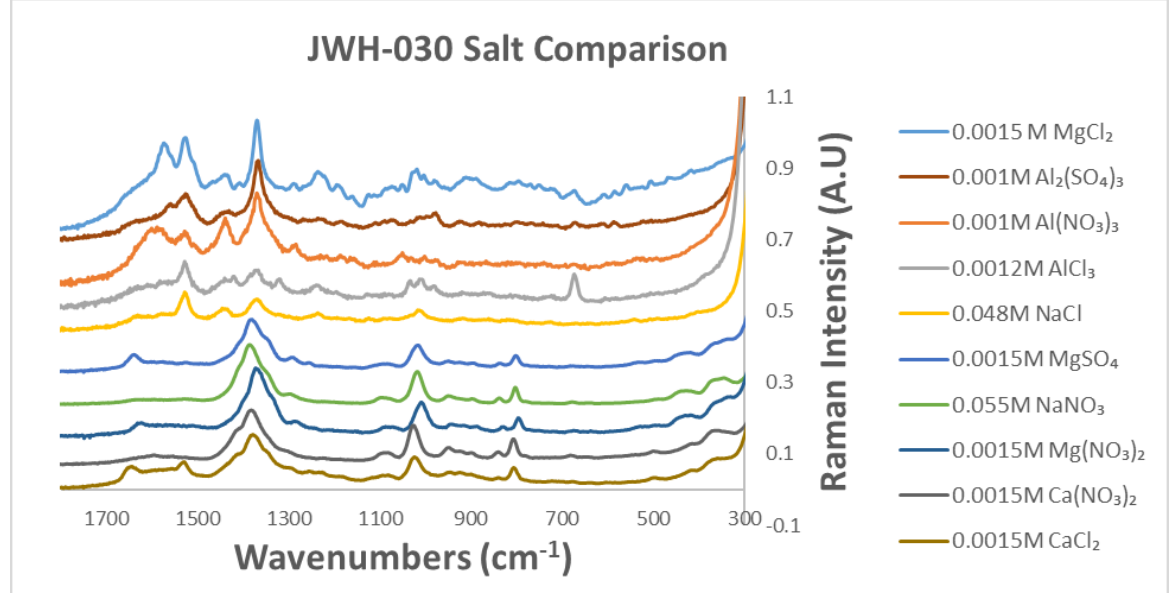

Figure 19: A comparison of the SERS spectrum of (A) JWH-122 at $100 \mathrm{ng} \mathrm{mL}^{-1}$ with various chloride salts to that of the library spectrum of JWH-122 from the portable Raman used. The solutions examined contained gold nanoparticles, chloride salts at the concentrations noted in Table 1 Row $\mathrm{C}$ and JWH-122 in $0.02 \mathrm{M}$ methanol solution. (B) JWH-030 at $100 \mathrm{ng} \mathrm{mL}^{-1}$ with various chloride, sulfate and nitrate salts. The solutions examined contained gold nanoparticles, various chloride, sulfate and nitrate salts at the concentrations noted in Table 1 Row C, and JWH-030 in $0.02 \mathrm{M}$ methanol solution. 


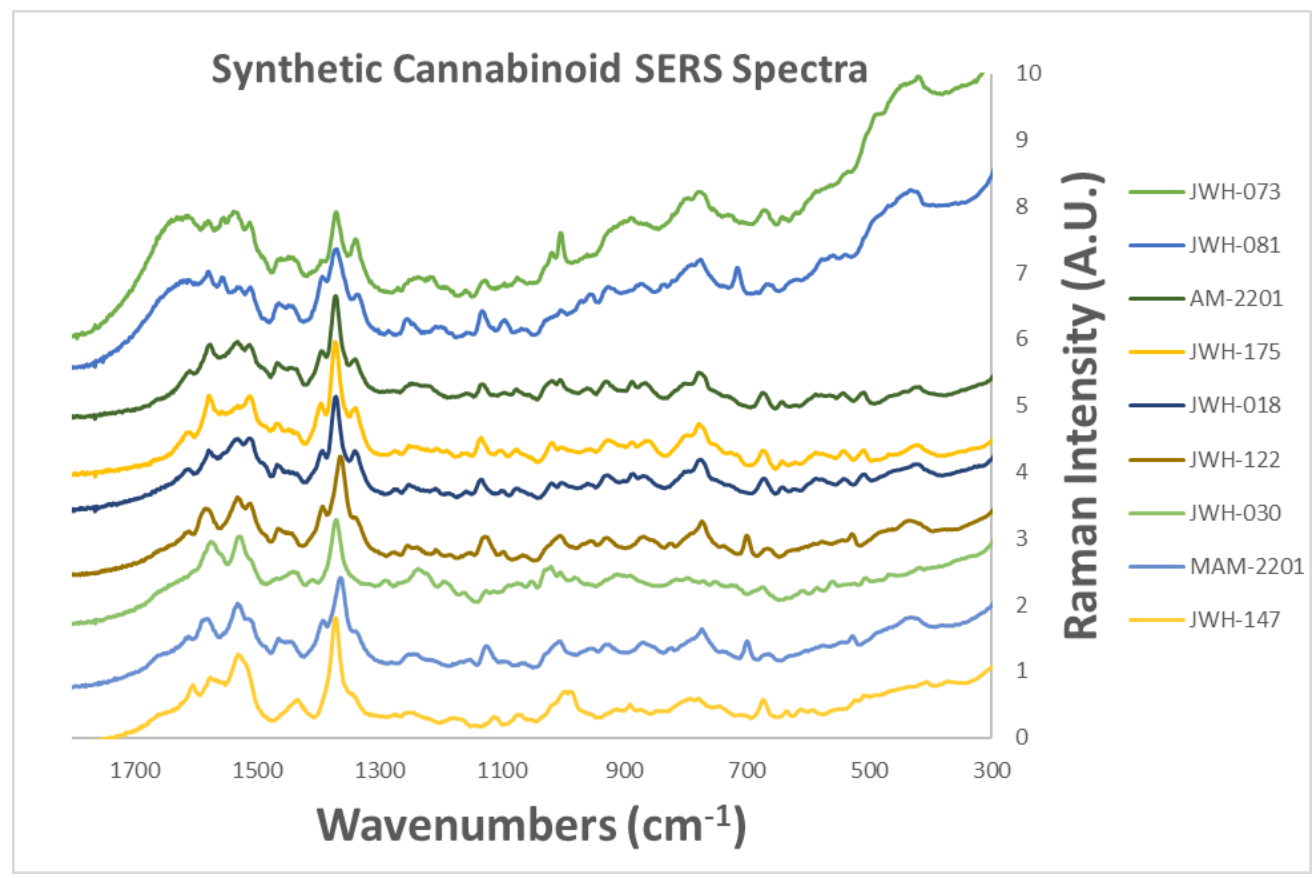

Figure 20: The SERS spectrum of all synthetic cannabinoids examined at $100 \mathrm{ng} \mathrm{mL}^{-1}$ in $0.02 \mathrm{M}$ methanol concentration using $0.0015 \mathrm{M} \mathrm{MgCl}_{2}$ as the aggregating agent.

\begin{tabular}{|c|lllll|}
\hline Drug & $\begin{array}{l}\text { Aggregating } \\
\text { Agent }\end{array}$ & Slope & $\begin{array}{l}\text { Standard } \\
\text { Deviation }\end{array}$ & $\mathbf{R}^{\mathbf{2}}$ & $\begin{array}{l}\text { LOD } \\
\text { (ng/mL) }\end{array}$ \\
\hline JWH-018 & $\mathrm{CaCl}_{2}$ & 0.00007 & 0.025 & 0.75 & 1090 \\
& $\mathrm{MgCl}_{2}$ & 0.0007 & 0.0005 & 0.89 & 20 \\
JWH-030 & $\mathrm{MgCl}_{2}$ & 0.0005 & 0.011 & 0.99 & 68 \\
& $\mathrm{AlCl}_{3}$ & 0.000007 & 0.008 & 0.96 & 3313 \\
JWH-073 & $\mathrm{NaCl}$ & 0.00001 & 0.0007 & 0.94 & 221 \\
& $\mathrm{MgCl}_{2}$ & 0.0007 & 0.03 & 0.98 & 130 \\
JWH-081 & $\mathrm{NaCl}$ & 0.000005 & 0.0005 & 0.83 & 328 \\
& $\mathrm{MgCl}_{2}$ & 0.0018 & 0.040 & 0.94 & 66 \\
JWH-122 & $\mathrm{MgCl}_{2}$ & 0.0018 & 0.023 & 0.97 & 38 \\
& $\mathrm{AlCl}_{3}$ & 0.00003 & 0.0058 & 0.98 & 583 \\
JWH-175 & $\mathrm{MgCl}_{2}$ & 0.0031 & 0.091 & 0.89 & 88 \\
& $\mathrm{AlCl}_{3}$ & 0.000003 & 0.0003 & 0.94 & 266 \\
& $\mathrm{CaCl}_{2}$ & 0.0003 & 0.028 & 0.83 & 746 \\
$\mathrm{MAM}-2201$ & $\mathrm{MgCl}_{2}$ & 0.0018 & 0.019 & 0.95 & 30 \\
& $\mathrm{MgCl}_{2}$ & 0.0009 & 0.008 & 0.95 & 27 \\
$\mathrm{AM}-2201$ & $\mathrm{AlCl}_{3}$ & 0.000004 & 0.0007 & 0.98 & 554 \\
& $\mathrm{NaCl}$ & 0.000006 & 0.00007 & 0.96 & 334 \\
& $\mathrm{MgCl}_{2}$ & 0.001 & 0.038 & 0.99 & 113 \\
& $\mathrm{AlCl}_{3}$ & 0.00002 & 0.010 & 0.91 & 1463 \\
\hline
\end{tabular}

Table 7: The aggregating agent used along with the calculated slope, standard deviation, $\mathrm{R}^{2}$, and limits of detection to determine the optimal aggregating agent to use with the synthesized gold nanoparticles to detect 9 different synthetic cannabinoids in solution. 


\subsection{Conclusion}

An analysis of the gold nanoparticle and aggregating agent interaction was performed by accessing the change in absorbance, nanoparticle size, and nanoparticle zeta potential with monovalent, divalent, and trivalent chloride, sulfate, and nitrate salts. Results showed that divalent and trivalent cations achieve the CCC at lower concentrations compared to monovalent cations. Data from Zetasizer measurements indicate that the observed color changes are the result of the formation of larger aggregates of nanoparticles with an average size that increases with added salt concentration. There is also a corresponding reduction in charge indicating adsorption of cations following salt addition. Our results demonstrate the strong effect of divalent and trivalent cations in producing aggregation. The effects are independent of ionic strength and appear to correspond to the Hoffmeister effects. When used to examine the SERS effect on cannabinoids which are generally compounds, di and trivalent cations produce aggregation at the lowest concentration. This can be an important advantage for SERS measurements as these lower concentrations reduce background signal.

The optimal SERS procedure involved the use of $\mathrm{MgCl}_{2}$ as the aggregating agent to detect nine synthetic cannabinoids with limits of detection ranging from 20-130 ng $\mathrm{mL}^{-1}$ using a portable Raman system. Even lower detection limits may be obtained using a benchtop system. ${ }^{87}$ This developed SERS method is simple, sensitive and should be a useful technique for screening toxicological samples. 


\section{THE INITIAL ASSESSMENT OF SERS FOR THE TOXICOLOGICAL}

ANALYSIS OF SYNTHETIC CANNABINOID METABOLITES IN SPIKED URINE

\section{SAMPLES}

\subsection{Abstract}

As more and more synthetic cannabinoids are discovered and become scheduled, newer compounds having similar effects on the $\mathrm{CB}_{1}$ and $\mathrm{CB}_{2}$ receptors are being developed. In this new normal, a method needs to be developed to detect the current synthetic cannabinoids and their metabolites as well as those to be developed in the future. The current workhorse screening method for the detection of these compounds and their metabolites are immunoassays.

While these immunoassays have cross-reactivity and can detect compounds with a similar chemical structure, as newer compounds with different chemical structures are created newer immunoassays need to be developed. This can take a long time. However, SERS can be used to detect the current synthetic cannabinoids on the market and those in created in the future.

Upon the development of an optimized SERS method for the detection of pure synthetic cannabinoid parent compounds, a study was conducted the on the detection of two synthetic cannabinoid metabolites in spiked urine samples. Each spiked urine sample was extracted using SLE or $\mu$-SPE followed by SERS. While the JWH-018 metabolites could not be detected using SLE, they could be detected using $\mu$-SPE.

\subsection{Introduction}

SERS In recent years there has been an emergence in the development, use, and abuse of novel psychoactive substances (NPS). One subgroup of these substances are 
synthetic cannabinoids, which produce effects on the $\mathrm{CB}_{1}$ and/or $\mathrm{CB}_{2}$ receptors to mimic the effects of naturally occurring cannabinoids. ${ }^{2,3}$ Initially, these compounds were studied as legal alternative to the naturally occurring and controlled delta-9-THC. Currently, synthetic cannabinoids are schedule I controlled substances in the United States.

Toxicologically, a variety of immunoassays ${ }^{12-15,18}$ and LC-MS ${ }^{12,15,19,21-23}$ methods have been developed to detect the current synthetic cannabinoid compounds being used. As newer synthetic cannabinoids are being synthesized, newer immunoassays need to be developed to detect these compounds. Immunoassays can be a great tool in toxicology to combat this issue due to their cross-reactivity, detecting a wide range of compounds with a similar chemical structure. However, not all synthetic cannabinoids have the same base chemical structure that produce the antibody antigen interaction needed to see a color change to detect future compounds of interest. There are currently developed methods that can be used to combat this threat, such as surface enhanced Raman spectroscopy (SERS).

SERS uses metallic nanoparticles along with aggregating agents to be able to detect compounds at low $\mathrm{ng} / \mathrm{mL}$ concentrations. Previous work from my research group has demonstrated the detection of various synthetic cannabinoids in oral fluids using SERS. JWH-018 was detected in spiked oral fluid with a LOD of $0.8 \mathrm{ng} / \mathrm{mL}$ in using a $\mu$ SPE method consisting of C-18 stationary phase packed within ZipTip pippettes. ${ }^{87}$ However, to date, little work has been performed on the detection of synthetic cannabinoid metabolites in urine samples using SERS.

Another important factor in the detection of synthetic cannabinoids in biological samples is the extraction method used. One such extraction method is supported liquid 
extraction, (SLE). In SLE, the aqueous media is supported by diatomaceous earth, a polar support material. In the extraction process, non-polar drugs and metabolites move to the surface of the aqueous phase where they can be captured by a nonpolar organic solvent. ${ }^{99}$ This method is fast and does not require any column pretreatment like SPE. In total, from extraction to analysis can be done in less than 40 minutes per sample, including sample prep, extraction, and analysis. ${ }^{45}$ Previously, our research group developed an optimal SERS method for the detection of nine synthetic cannabinoid parent compounds. ${ }^{100}$ Gold nanoparticles and $\mathrm{MgCl}_{2}$ along with SLE have been used to detect 11 different benzodiazepines spiked in urine samples with limits of detection ranging from 0.5 to 120 $\mathrm{ng} / \mathrm{mL} .{ }^{45}$

Another extraction method examined utilized $\mu$-SPE. The method used a $10 \mu \mathrm{L}$ ZipTip pipette tip with $0.6 \mu \mathrm{L}$ of a $\mathrm{C} 18$ reverse phase particles. The procedure is convenient and can be completed in less than 36 minutes of total analysis time. ${ }^{87}$ It is capable of detecting JWH-018 in spiked oral fluid samples with a LOD of $0.8 \mathrm{ng} / \mathrm{mL} .{ }^{87}$

The goal of this study was to see if SLE or a ZipTip extraction method could be combined with SERS could be used to detect synthetic cannabinoid metabolites in spiked urine samples. Synthetic cannabinoid metabolites were examined in this part of the study since the parent compounds are not commonly found in urine samples. This study was split into two parts. First, the optimal SERS method developed in the previous chapter for synthetic cannabinoid parent compounds was used to detect JWH-018 N-pentanoic acid and JWH-018 N-(5-hydroxypentyl) metabolites (Figure 18) diluted in 10\% methanol. This Raman spectrum was set as a baseline to determine if the synthetic cannabinoid could be extracted using SLE or ZipTip. The second part of this study focused on the two 
extraction methods. In SLE, a few sample pretreatments and one post treatment method were compared in order to optimize the detection of two JWH-018 metabolites in spiked urine samples. The ZipTip extraction method was similar to what was previously developed for cannabinoids in saliva. ${ }^{87}$ After a variety of samples examined, JWH-018 N-pentanoic acid and JWH-018 N-(5-hydroxypentyl) metabolites could not be detected in spiked urine samples via SLE and SERS, but could be detected using the ZipTip extraction method.

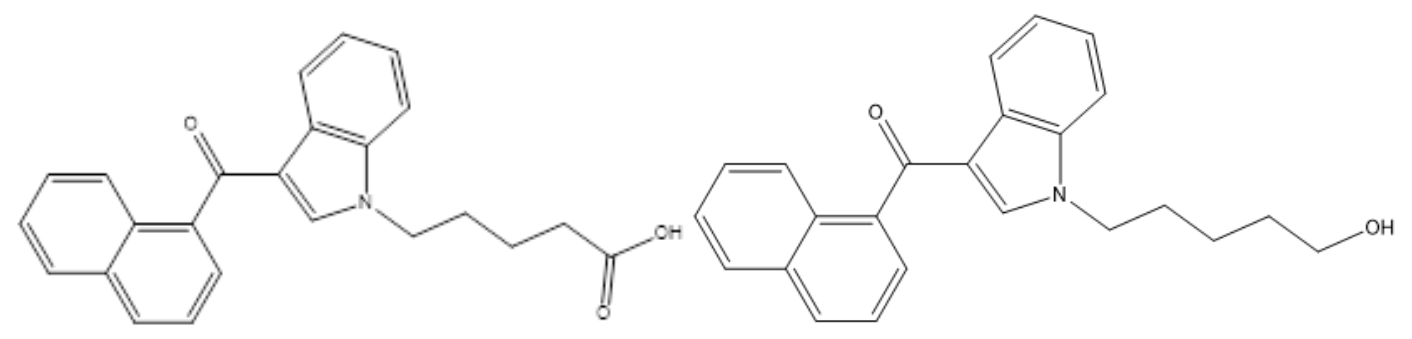

Figure 21: The chemical structures of JWH-018 N-pentanoic acid metabolite (left) and JWH-018 N-(5-hydroxypentyl) metabolite (right) examined in this study.

\subsection{Experimental}

\subsubsection{Materials used}

The gold nanoparticle colloids were prepared following the same procedure seen in chapters 3 and $4.100 \mathrm{~mL}$ gold nanoparticle solutions were prepared reducing tetrachloroauric (iii) acid trihydrate (Acros Organics, LOT\#A0354619) with $4 \mathrm{~mL}$ of a $1 \%$ by weight solution of sodium citrate dihydrate (Fisher Scientific, LOT \#091236) at $\mathrm{pH}$ 5. A stock solution of $0.15 \mathrm{M} \mathrm{MgCl}_{2}$ (Fisher Scientific) was used as the aggregating agent in this study.

Synthetic cannabinoid standards of JWH 018 N-(5-hydroxypentyl) metabolite (LOT \#0448993-60) and JWH-018 N-pentanoic acid metabolite (LOT \#0498078-28) 
were obtained from Cayman Chemical. Both standards were used to create stock standard solutions at concentrations ranging from $25-20000 \mathrm{ng} / \mathrm{mL}$ in a $10 \%$ methanol solution.

\subsubsection{SLE+ extraction}

The SLE method examined is similar to the methods examined by Doctor et al. ${ }^{45}$ Spiked urine samples of JWH 018 N-(5-hydroxypentyl) metabolite and JWH-018 Npentanoic acid metabolite in $10 \%$ methanol were analyzed. In this process, $500 \mu \mathrm{L}$ of the synthetic cannabinoid metabolite and $500 \mu \mathrm{L}$ of urine were added to a clear $4 \mathrm{~mL}$ glass vial (Fisher Scientific) and vortexed. Then, either $1 \mathrm{~mL}$ of DI water, $1 \mathrm{~mL}$ of $50 \mathrm{mM}$ $\mathrm{NH}_{4} \mathrm{OAc}$ buffer solution (adjusted to $\mathrm{pH} 2$ with $1 \%$ formic acid), or $1 \mathrm{~mL}$ of $50 \mathrm{mM}$ $\mathrm{NH}_{4} \mathrm{OAc}$ buffer solution (adjusted to $\mathrm{pH} 4$ with $1 \%$ formic acid) were added to the $4 \mathrm{~mL}$ glass vial and vortexed (total solution volume of $2 \mathrm{~mL}$ ) and applied to the Biotage Isolute supported liquid extraction (SLE)+ $2 \mathrm{~mL}$ column under vacuum and let to sit for five minutes. Next, a wash of $2.5 \mathrm{~mL}$ dichloromethane (Fisher Scientific Lot \#138217) was applied to the SLE+ column and let sit for five minutes. Then, another wash of $2.5 \mathrm{~mL}$ dichloromethane was applied to the SLE+ column and let sit for five minutes and eluted under vacuum for 10 seconds. Finally, the eluted dichloromethane was dried down under nitrogen gas, and reconstituted with $500 \mu \mathrm{L}$ of $10 \%$ methanol.

Some addition modifications were examined to see if the synthetic cannabinoid metabolites could be extracted. Some samples were treated with $100 \mu \mathrm{L}$ of acidified methanol prior to being dried down and reconstituted with $10 \%$ methanol. In addition, instead of two washes of $2.5 \mathrm{~mL}$ dichloromethane applied to the SLE column, one wash of $2 \mathrm{~mL}$ dichloromethane was examined. 


\subsubsection{Micro-SPE extraction}

The $\mu$-SPE extraction method using ZipTip pipette tips is similar to the method developed by Deriu et al. ${ }^{87}$ For this method, $150 \mu \mathrm{L}$ of spiked urine samples containing JWH $018 \mathrm{~N}$-(5-hydroxypentyl) metabolite and JWH-018 N-pentanoic acid metabolite in pure methanol were added to $250 \mu \mathrm{L}$ of urine $(400 \mu \mathrm{L}$ total volume, with $37.5 \% \mathrm{v} / \mathrm{v}$ methanol) and centrifuged for 10 minutes at speed of 12.4 revolutions per minute. Then, $49.5 \mu \mathrm{L}$ of the spiked urine sample was removed and placed in a $2 \mathrm{~mL}$ glass vial (Fisher Scientific) with the addition of $0.5 \mu \mathrm{L}$ of DI water adjusted to $\mathrm{pH} 2.5$ with formic acid (Acros Organics, lot \# A0022334000) and vortexed.

After ample preparation, the ZipTip $10 \mu \mathrm{L}$ pipet tips (Millipore, lot \# R7EA90580), fitted with a $0.6 \mu \mathrm{L}$ reversed phase $\mathrm{C} 18$ chromatographic bed with no dead volume was conditioned with two washes of $10 \mu \mathrm{L}$ of $100 \%$ acetonitrile (UHPLC/UV grade, Fisher Chemical, lot \#181464) followed by two washes of $10 \mu \mathrm{L}$ of DI water acidified to $\mathrm{pH} 2.5$ via addition of formic acid at a final concentration of $1 \% \mathrm{v} / \mathrm{v}$. Then, the ZipTip was washed ten times with $10 \mu \mathrm{L}$ of the urine sample with formic acid. Finally, the ZipTip was washed with $4 \mu \mathrm{L}$ of the methanol and eluted into a $2 \mathrm{~mL}$ glass vial.

\subsubsection{SERS analysis of metabolites}

The SLE treated samples followed the procedure previously explained in chapters 3 and 4 of this work: $980 \mu \mathrm{L}$ of the gold nanoparticle solution and $10 \mu \mathrm{L}$ of $0.15 \mathrm{MgCl}_{2}$ solution were placed in a clear $2 \mathrm{~mL}$ glass vial (Fisher Scientific) vortexed for 30 seconds followed by the addition of $10 \mu \mathrm{L}$ of the pure metabolites or the extracted metabolites and vortexed for 30 seconds. The $\mu$-SPE method followed a similar procedure to Deriu et al. ${ }^{87}$ 
After $4 \mu \mathrm{L}$ of methanol was eluted from the ZipTip, then $245 \mu \mathrm{L}$ of gold nanoparticle solution was added and vortexed for 30 seconds followed by $2.5 \mu \mathrm{L}$ of $0.15 \mathrm{MgCl}_{2}$. The concentration of spiked urine samples ranged from 25-20000 ng/mL. Each sample was run in triplicate. The final concentration of each component in the for the pure and SLE samples was as follows: $0.05 \mathrm{mM} \mathrm{HAuCl}_{4}, 0.0015 \mathrm{M} \mathrm{MgCl}_{2}$, and between 2.5-2000 $\mathrm{ng} / \mathrm{mL}$ of pure or extracted metabolites. The final concentration of each component in the $\mu$-SPE samples was as follows: $0.05 \mathrm{mM} \mathrm{HAuCl}_{4}, 0.0015 \mathrm{M} \mathrm{MgCl}_{2}$, and between 9.4$7500 \mathrm{ng} / \mathrm{mL}$ of extracted metabolites.

\subsubsection{Instrumentation}

The extracted SERS solutions were transferred to a quartz Suprasil@ cuvette (Hellma Analytics) and analyzed via the Thermo Scientific FirstDefenderRM (portable) with a $785 \mathrm{~nm}$ laser at high power $(250 \mathrm{~mW})$ with a resolution of 7 to $10.5 \mathrm{~cm}^{-1}$ (FWHM).

\subsection{Results and discussion}

\subsubsection{SERS analysis of metabolite standards}

In this study, metabolites of JWH-018 were examined. The goal of this study was to develop a fast and easy extraction method that could be used to detect synthetic cannabinoids in urine. Initially, the synthetic cannabinoid standards were analyzed via the optimized SERS method developed in chapter 4. The SERS spectrum of each cannabinoid metabolite ranging between $2.5-20000 \mathrm{ng} / \mathrm{mL}$ can be seen in Figure 22. The results show that these compounds can be detected at concentrations as low as 2.5$10 \mathrm{ng} / \mathrm{mL}$. 

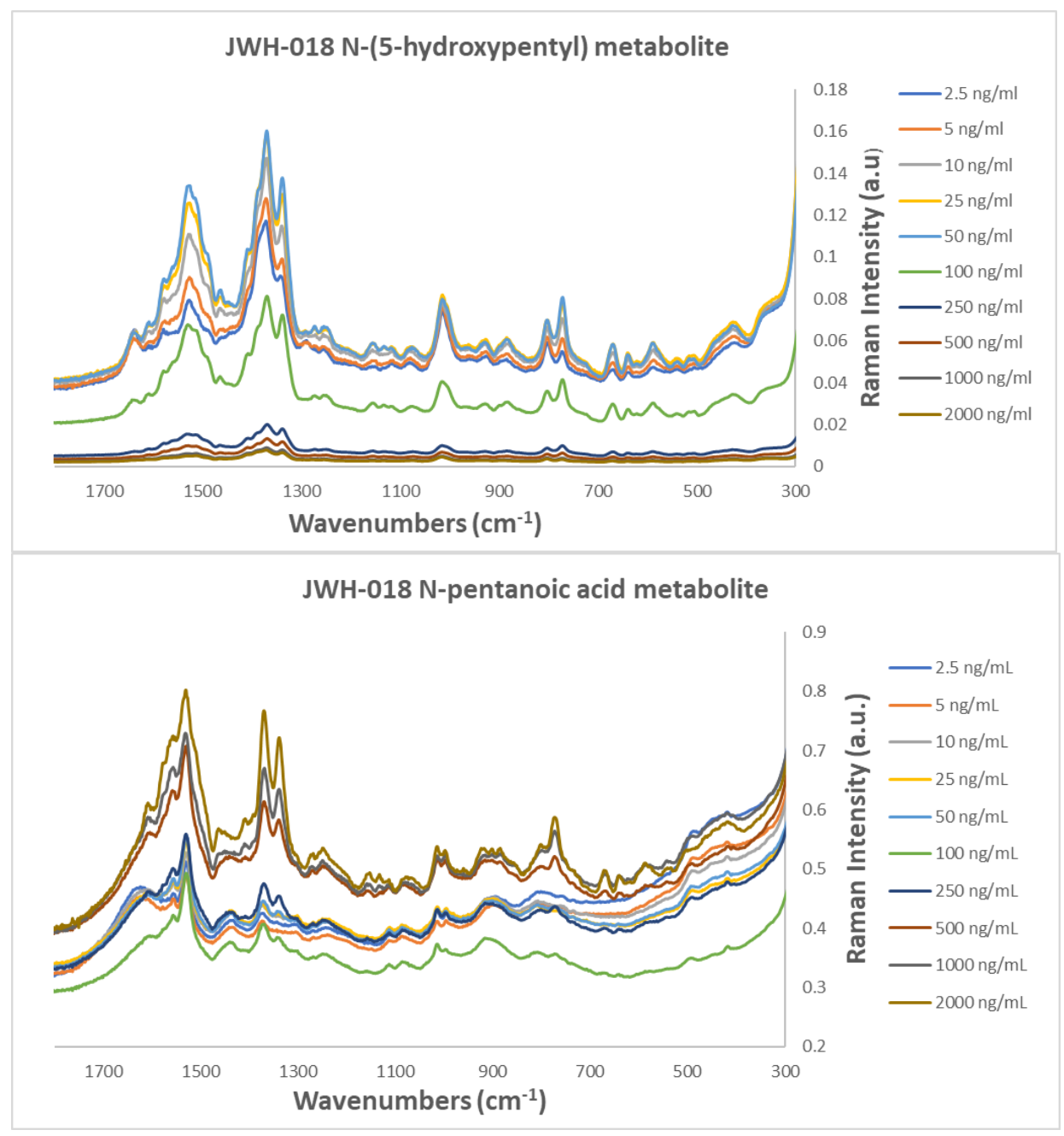

Figure 22: The SERS spectrum of JWH-018 N-(5-hydroxypentyl) metabolite ranging from 2.5-2000 ng/mL (top) and The SERS spectrum of JWH-018 N-pentanoic acid metabolite ranging from $2.5-2000 \mathrm{ng} / \mathrm{mL}$ (bottom).

In addition, the Raman intensity at a specific peak in the Raman spectra for each compound was plotted versus the concentration (Figure 23). The results demonstrate the expected Langmuir adsorption behavior and illustrate the capability of the method to detect physiological concentrations of the metabolites. A comparison of the SERS spectrum of the JWH-018 metabolites to the parent compound can be seen in Figure 24 . The SERS spectrum of the pure drug and the JWH metabolites are discussed in detail in chapter 6. 

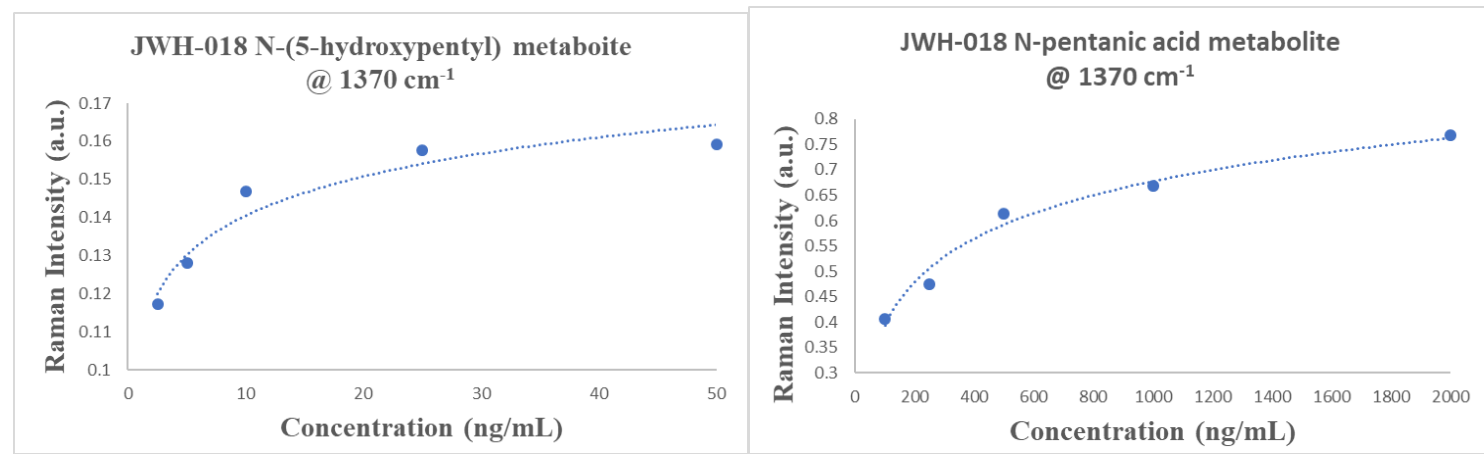

Figure 23: A plot of Raman intensity of a specific peak for each JWH-018 metabolite (pure drug) versus the concentration.

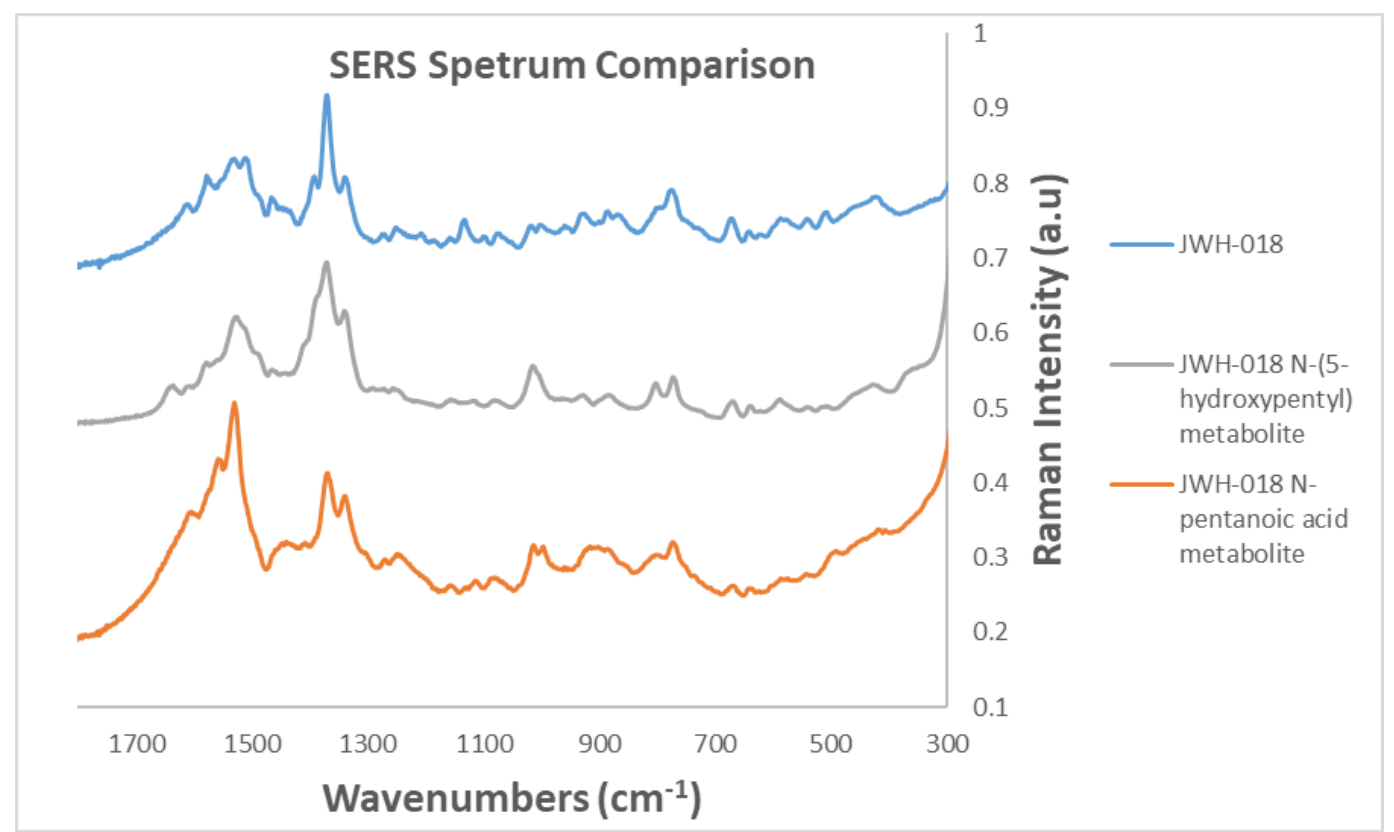

Figure 24: Comparison of the SERS spectrum of JWH-018 (100 ng/mL), JWH-018 (N-5hydroxypentyl) metabolite $(100 \mathrm{ng} / \mathrm{mL})$, and JWH-018 N-pentanoic acid metabolite (500 $\mathrm{ng} / \mathrm{mL}$ ) in $10 \%$ methanol.

\subsubsection{SLE+ extraction of synthetic cannabinoids}

After the metabolite standards were analyzed via the optimized SERS method in chapter $4,500 \mu \mathrm{L}$ of each metabolite concentration was spiked to $500 \mu \mathrm{L}$ of urine and extracted. Prior to adding the spiked urine sample to the SLE column samples were pretreated with $1 \mathrm{~mL}$ of DI water, $1 \mathrm{~mL}$ of $50 \mathrm{mM} \mathrm{NH}_{4} \mathrm{OAc}$ buffer solution (adjusted to $\mathrm{pH} 2$ with $1 \%$ formic acid), or $1 \mathrm{~mL}$ of $50 \mathrm{mM} \mathrm{NH} 4 \mathrm{OAc}$ buffer solution (adjusted to $\mathrm{pH} 4$ 
with $1 \%$ formic acid). The resultant spectra could not be distinguished from the negative control (Figure 25). Based upon the results, it was clear that the synthetic cannabinoid metabolites were lost in the extraction process, and an alternative extraction method was necessary.

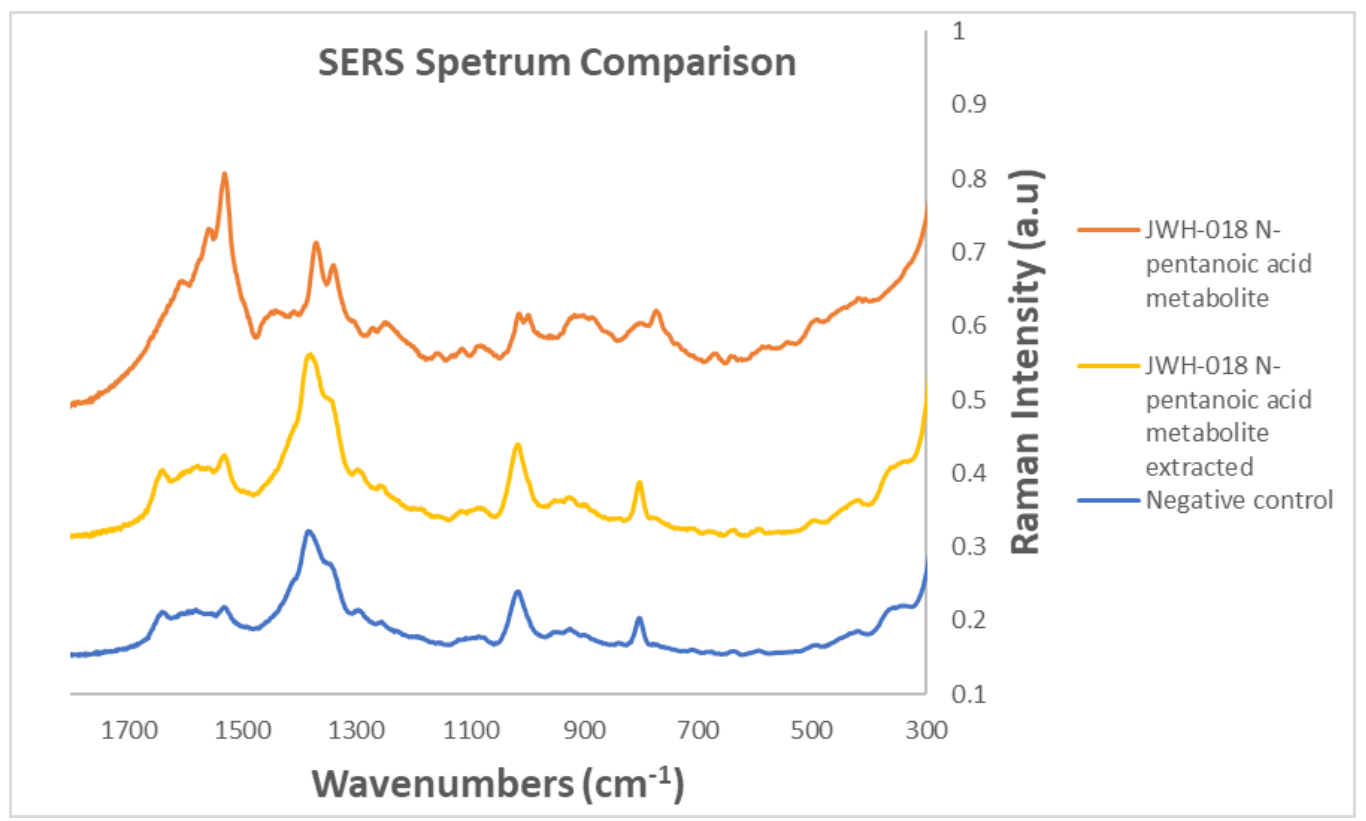

Figure 25: Comparison of the SERS spectrum of JWH-018 N-pentanoic acid metabolite $(500 \mathrm{ng} / \mathrm{mL})$ prior to extraction and JWH-018 N-pentanoic acid metabolite spiked urine sample $(1000 \mathrm{ng} / \mathrm{mL})$ pretreated with $1 \mathrm{~mL}$ of $50 \mathrm{mM} \mathrm{NH} \mathrm{NHAc}_{4} \mathrm{Ouffer}$ solution (adjusted to $\mathrm{pH} 4$ with $1 \%$ formic acid).

\subsubsection{Micro-SPE of synthetic cannabinoids}

To improve the extraction process, a $\mu$-SPE method was developed using $0.6 \mu \mathrm{L}$ of a reversed phase $\mathrm{C} 18$ chromatographic bed packed within a $10 \mu \mathrm{L}$ pipette tip. MicroSPE is completed in five steps: wetting, equilibration, binding, washing and elution. The entire process from sample to measurement takes under 36 minutes. ${ }^{87}$ The SERS spectrum of each compound after the $\mu$-SPE extraction is shown in Figure 26. The results show a clear distinction between the parent drug and the metabolites. In initial experiments examining the potential of $\mu$-SPE for sensitivity, the JWH-018 N-(5- 
hydroxypentyl) metabolite was detected at concentrations as low as $250 \mathrm{ng} / \mathrm{mL}$ and the JWH-018 N-pentanoic acid metabolite as low as $375 \mathrm{ng} / \mathrm{mL}$. These results demonstrate the potential of zip tip based solid phase extraction. Further experiments are necessary to optimize recovery and improve detection.

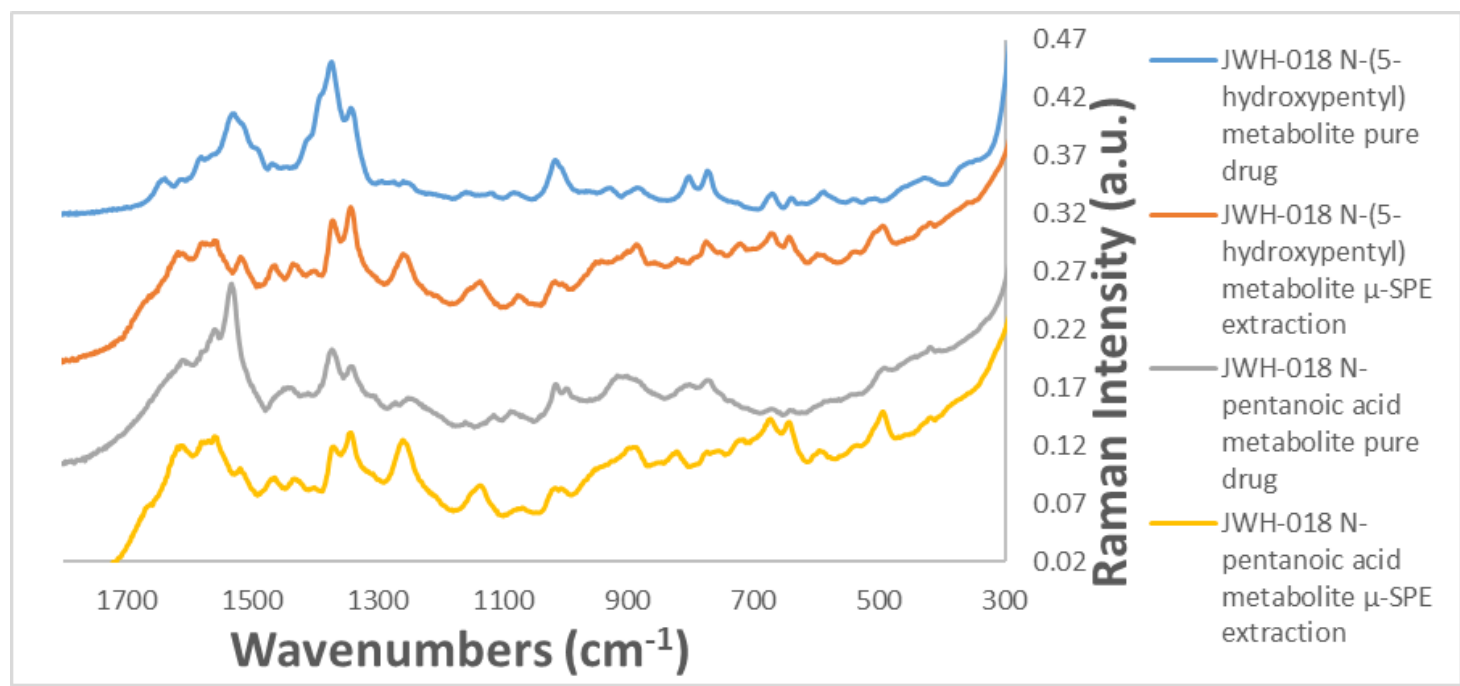

Figure 26: A comparison of the SERS spectrum of the JWH-018 N-(5-hydroxypentyl) metabolite as the pure drug $(100 \mathrm{ng} / \mathrm{mL})$ and after $\mu$-SPE extraction $(375 \mathrm{ng} / \mathrm{mL})$ as well as JWH-018 N-pentanoic acid metabolite as the pure drug $(250 \mathrm{ng} / \mathrm{mL})$ and after $\mu$-SPE (375 ng/mL).

\subsection{Conclusion}

The use of SLE and $\mu$-SPE with SERS was examined as a possible method to detect two JWH-018 metabolites in spiked urine samples. Prior to an extraction and spiking of urine samples, the metabolites were analyzed via the optimized SERS method developed in chapter 4, at concentrations as low as $2.5 \mathrm{ng} / \mathrm{mL}$. Initial analysis using SLE to extract the metabolites from the spiked urine samples did not produce a SERS spectrum different from the negative control. This could be due to matrix interferences in the urine samples or loss of sample during extraction. Initial results using $\mu$-SPE demonstrated the detection of the drug metabolites at concentrations as low as $250 \mathrm{ng} / \mathrm{mL}$. 
These results indicate that with further optimization, $\mu$-SPE should provide a useful method for the extraction JWH-018 metabolites in urine samples.

\section{VIBRATIONAL SPECTRAL ANALYSIS OF SYNTHETIC CANNABINOIDS}

\subsection{Introduction}

Spectroscopy measures vibrational frequencies produced from inter or intra molecular bond vibrations. The two types main types of molecular bond vibrations between atoms are stretching vibrations (symmetric and antisymmetric) and deformation vibrations (bending, wagging, twisting, and rocking). ${ }^{26}$ The two spectroscopic techniques that measure these vibrations are IR and Raman spectroscopy. Infrared spectroscopy is related to the change in the dipole moment of a molecule, whereas the Raman reflects the change in polarizability. Molecular bonds of a molecule can be either IR active, Raman active, or both.

A few articles have been published on the structural interpretation on the Raman spectrum of some synthetic cannabinoids. Deriu et al. used density functional theory (DFT) calculations to determine the vibrational assignments of JWH-018 in solid state and in oral fluid via SERS. ${ }^{87}$ Alkaseem et al. used DFT calculations to determine the vibrational assignments of 5F-PB-22 standard in solid state and in solution via SERS. The differences in the powder, SERS, and DFT calculated Raman spectrum were discussed ${ }^{50}$ In addition, a similar comparison of AMB-FUBINACA was conducted by

Islam et al. using DFT calculations to assign the major peaks in each Raman spectrum. ${ }^{49}$

The above articles were used to identify the various peaks in the SERS spectrum of the synthetic cannabinoid parent and metabolite compounds examined in this study. 
The study by Deriu et al was used to assign the peaks of JWH-018. Since the additional parent and metabolite compounds were similar in structure, the peak assignments of the additional compounds were based on the initial assignments of JWH-018. For the fluorinated synthetic cannabinoid compounds examined, the carbon fluorine single bond assignment was based on the work of Alkaseem et al. In this spectral interpretation, the strength of the bands as well as the stretching and deformation vibrations are noted. The band strength is noted as follows: strong (s), moderate (m), weak (w), and very weak (vw).

\subsection{Analysis of Peaks for All synthetic cannabinoids and Major Subgroups}

The structure of the synthetic cannabinoids examined in this study are aminoalkylindoles (Figure 1) that can be broken down into 4 major groups: core group, linker, linked group and tail. ${ }^{87}$ (Figure 27). The core group is the indole. The linker is a ketone. The linked group is naphthalene. The tail is an alkane chain. The SERS spectrum with the chemical structure as well as peak identification of JWH-018 can be seen in Figure 28 and Table 8.

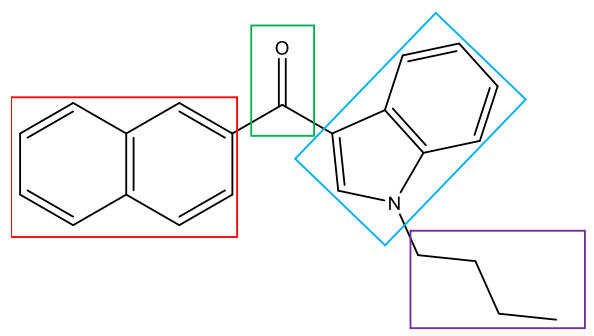

Figure 27: The identification of the four major groups of aminoalkylindoles (JWH-073): linked group (red), linker (green), core group (blue), and tail (purple). ${ }^{87}$ 


\begin{tabular}{|c|c|}
\hline Wavenumber & Assignment \\
\hline $1610 \mathrm{w}$ & $\mathrm{C}=\mathrm{C}$ stretching indole rings \\
\hline $1573 \mathrm{w}$ & $\mathrm{C}=\mathrm{C}$ stretching naphthalene ring \\
\hline $1530 \mathrm{w}$ & $(\mathrm{C}=\mathrm{C}) \mathrm{CCN}$ stretching; $(\mathrm{CH}) \mathrm{NCH}_{2}$ in plane deformation \\
\hline $1509 \mathrm{w}$ & $(\mathrm{C}=\mathrm{C}) \mathrm{CCN}$ stretching; $(\mathrm{CH}) \mathrm{NCH}_{2}$ in plane deformation \\
\hline $1463 \mathrm{w}$ & $\begin{array}{l}\mathrm{CH}_{2} \text { scissoring; } \mathrm{CH}_{3} \text { scissoring within indole ring; } \mathrm{CH} \text { scissoring within } \\
\text { naphthalene ring }\end{array}$ \\
\hline $1393 \mathrm{w}$ & $\mathrm{CH}$ in plane rocking within naphthalene ring \\
\hline $1370 \mathrm{~s}$ & $\mathrm{C}=\mathrm{C}$ stretching in naphthalene ring \\
\hline $1338 \mathrm{w}$ & $\begin{array}{l}\mathrm{C}=\mathrm{C} \text { stretching within indole ring; } \mathrm{CH} \text { in plane scissoring within indole or } \\
\text { naphthalene ring }\end{array}$ \\
\hline $1272 \mathrm{w}$ & $\mathrm{CH}_{2}$ out of plane twisting; $\mathrm{CH}$ in plane scissoring in indole and naphthalene ring \\
\hline $1251 \mathrm{w}$ & $\begin{array}{l}\mathrm{HCH} \text { out of plane wagging form tail; } \mathrm{CH} \text { in plane scissoring within the indole or } \\
\text { naphthalene ring }\end{array}$ \\
\hline $1207 \mathrm{vw}$ & $\begin{array}{l}\mathrm{CH} \text { in plane scissoring in naphthalene ring; } \mathrm{CH}_{2} \text { out of plane twisting, } \mathrm{CH}_{3} \text { out of } \\
\text { plane twisting in the tail }\end{array}$ \\
\hline $1183 \mathrm{vw}$ & $\begin{array}{l}\mathrm{CH} \text { in plane scissoring in indole ring; } \mathrm{CH} \text { in plane rocking of the indole and } \\
\text { naphthalene ring }\end{array}$ \\
\hline $1160 \mathrm{vw}$ & $\mathrm{HCCH}$ in plane stretching on the indole and naphthalene ring \\
\hline $1134 \mathrm{~m}$ & $(\mathrm{CH}) \mathrm{NCH}$ in plane scissoring \\
\hline $1096 \mathrm{vw}$ & $\begin{array}{l}\mathrm{HCCH} \text { naphthalene ring in plane scissoring; } \mathrm{CH}_{2} \text { out of plane twisting; } \mathrm{CH}_{3} \text { out of } \\
\text { plane wagging on the tail }\end{array}$ \\
\hline $1074 \mathrm{w}$ & HCCH naphthalene ring in plane scissoring \\
\hline $1017 \mathrm{vw}$ & Indole ring breathing; $\mathrm{CC}$ stretching within the tail \\
\hline $1000 \mathrm{vw}$ & Indole ring breathing; $\mathrm{CC}$ stretching within the tail \\
\hline $960 \mathrm{w}$ & $\mathrm{CH}$ Naphthalene ring or of plane wagging \\
\hline $931 \mathrm{w}$ & $\mathrm{CH}$ out of plane wagging within the naphthalene ring \\
\hline $886 \mathrm{vw}$ & $\mathrm{CCH}_{3}$ stretching; in plane deformation within the idole ring or naphthalene ring \\
\hline $867 \mathrm{vw}$ & $\mathrm{CCH}_{3}$ stretching; in plane deformation within the idole ring or naphthalene ring \\
\hline $775 \mathrm{~m}$ & Indole ring breathing; $\mathrm{CH}$ out of plane wagging within naphthalene ring \\
\hline $672 \mathrm{w}$ & In plane deformation within naphthalene or indole ring \\
\hline $640 \mathrm{w}$ & In plane deformation within naphthalene ring \\
\hline $619 \mathrm{vw}$ & In plane deformation within naphthalene ring \\
\hline $589 \mathrm{w}$ & $\begin{array}{l}\text { In plane deformation within naphthalene ring and out of plane deformation indole } \\
\text { ring }\end{array}$ \\
\hline $541 \mathrm{w}$ & In plane deformation within naphthalene or indole ring \\
\hline $510 \mathrm{w}$ & In plane deformation within naphthalene ring \\
\hline $421 \mathrm{w}$ & $\mathrm{CH}$ out of plane wagging in naphthalene ring; Indole ring in plane deformation \\
\hline
\end{tabular}

Table 8: Vibrational assignment of peaks in the SERS spectrum of JWH-018. 


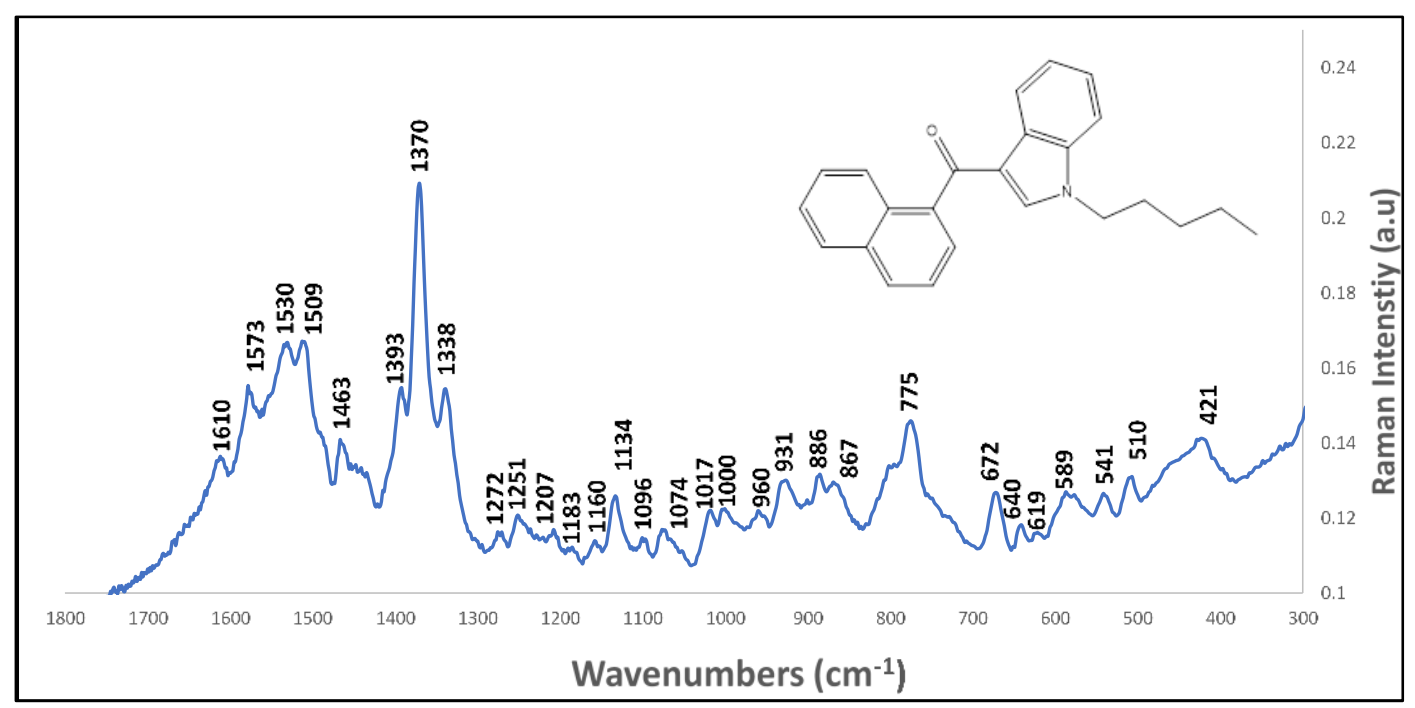

Figure 28: Chemical structure and SERS spectrum of JWH-018

All the SERS spectra discussed in this chapter were obtained using the Thermo Scientific First Defender RM (portable) with a $785 \mathrm{~nm}$ laser at high power $(250 \mathrm{~mW})$ with a resolution of 7 to $10.5 \mathrm{~cm}^{-1}$ (FWHM). Upon initial visualization of each synthetic cannabinoid examined, there are differences in the peak shape. However, due to the resolution of the instrument used, it cannot be stated that each compound produced a unique Raman spectrum that can be identified from each other. Instead, this research has shown that compounds of a similar chemical structure will have similarities in the Raman spectrum. Having this knowledge is important when attempting to do structural elucidation of an unknown compound using this method.

There are many peaks present in the SERS spectrum that could be due to more than one type of molecular bond vibration within the chemical structure of the synthetic cannabinoids examined in this study. However, there is a portion of the chemical structure missing in the SERS spectrum of all the parent compounds, except JWH-175, and metabolites examined. There is no peak around the $1650 \mathrm{~cm}^{-1}$ region of the SERS spectrum, which would be related to the ketone portion of the chemical structure. This 
peak is the linker portion of the molecule. In addition, there are portions of the metabolites chemical structure missing from the SERS Spectrum. Each metabolite is missing a broad peak from $\mathrm{OH}$ bond stretching, seen between $3000-3600 \mathrm{~cm}^{-1}$ and the pentanoic acid metabolite is missing a peak from the $\mathrm{C}=\mathrm{O}$ stretching of the carboxylic acid portion of the chemical structure around $1750 \mathrm{~cm}^{-1}$. These peak identifications are based upon the studies conducted by Munchi et al. on identification of GHB and GBL and Sun et al. on the $\mathrm{OH}$ stretching vibration of ice. ${ }^{101,102}$ One reason for not seeing a broad $0 \mathrm{H}$ stretching peak between $3000-3600 \mathrm{~cm}^{-1}$ is the limitation of the scan range of the portable Raman system, which reached only as far as $2900 \mathrm{~cm}^{-1}$. A larger scan range could increase the chance of seeing the SERS peak. However, another limitation to the SERS analysis is the rotation of the molecule present in the nanoparticle solution and the angle at which the incident light (laser) hits the sample to produce the SERS enhancement. Therefore, a portion of the chemical structure could be missing from the SERS spectrum. This could explain the variations in the SERS spectrum between the pure drug of the metabolites and after $\mu$-SPE of spiked urine samples. For a detailed analysis of the additional compounds examined see Chapter 6.3.

\subsection{Spectral Analysis of additional synthetic cannabinoids examined}

The SERS spectrum of each synthetic cannabinoid parent and metabolite examined along with the chemical structure of each compound can be seen in the figures below. The identification of each peak in the corresponding Raman spectrum can be seen in the tables below. 


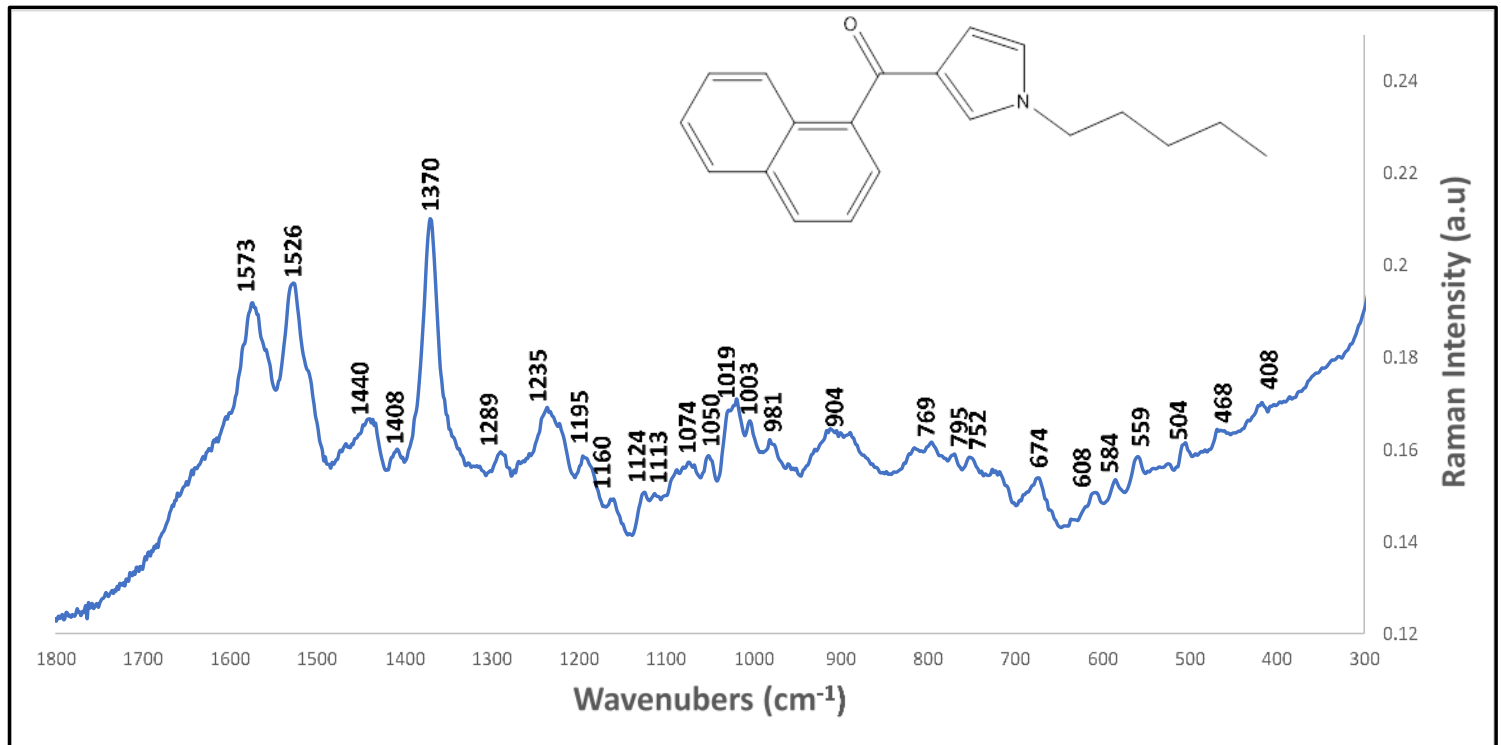

Figure 29: Chemical structure and SERS spectrum of JWH-030.

\begin{tabular}{|c|c|}
\hline Wavenumber & Assignment \\
\hline $1573 \mathrm{~m}$ & $\mathrm{C}=\mathrm{C}$ stretching naphthalene ring \\
\hline $1526 \mathrm{~m}$ & $(\mathrm{C}=\mathrm{C}) \mathrm{CCN}$ stretching; $(\mathrm{CH}) \mathrm{NCH} 2$ out of plane deformation \\
\hline $1440 \mathrm{~m}$ & $\mathrm{CH}$ out of plane rocking in the naphthalene ring \\
\hline $1408 \mathrm{w}$ & $\mathrm{CH}$ in plane rocking in the naphthalene ring \\
\hline $1370 \mathrm{~s}$ & $\mathrm{C}=\mathrm{C}$ stretching in naphthalene ring \\
\hline $1289 \mathrm{w}$ & $\mathrm{CH}_{2}$ out of plane twisting; $\mathrm{CH}$ in plane scissoring for indole and naphthalene ring \\
\hline $1235 \mathrm{~m}$ & $\begin{array}{l}\mathrm{HCH} \text { out of plane wagging form tail; } \mathrm{CH} \text { in plane scissoring within the idole or naphthalene ring } \\
\mathrm{CH} \text { in plane scissoring on pyrrole ring; } \mathrm{CH} \text { in plane rocking on pyrrole and naphthalene ring }\end{array}$ \\
\hline $1195 \mathrm{w}$ & \\
\hline $1160 \mathrm{w}$ & $\mathrm{HCCCH}$ in plane scissoring of naphthalene ring \\
\hline $1124 \mathrm{vw}$ & $(\mathrm{CH}) \mathrm{NCH}$ in plane scissoring \\
\hline $1113 \mathrm{vw}$ & $\begin{array}{l}\mathrm{CH}_{2} \text { out of plane wagging, } \mathrm{CH}_{3} \text { out of plane wagging on the tail; } \mathrm{CH} \text { in plane scissoring on the } \\
\text { naphthalene ring }\end{array}$ \\
\hline $1074 \mathrm{w}$ & $\mathrm{HCCCH}$ in plane scissoring of naphthalene ring \\
\hline $1050 \mathrm{w}$ & $\mathrm{C}-\mathrm{C}$ tail stretching \\
\hline $1019 \mathrm{w}$ & Pyrrole ring breathing; $\mathrm{C}-\mathrm{C}$ tail stretching \\
\hline $1003 \mathrm{vw}$ & Pyrrole ring breathing; $\mathrm{C}-\mathrm{C}$ tail stretching \\
\hline $981 \mathrm{vw}$ & $\mathrm{CH}$ naphthalene ring out of plane wagging \\
\hline $904 \mathrm{w}$ & $\mathrm{CCH}_{3}$ stretching; in plane deformation within the idole ring or naphthalene ring \\
\hline $795 \mathrm{vw}$ & $\mathrm{CH}_{2}$ in plane rocking; $\mathrm{CH}_{3}$ in plane rocking; $(\mathrm{CH}) \mathrm{NCH}$ out of plane wagging \\
\hline $769 \mathrm{vw}$ & Indole ring breathing; $\mathrm{CH}$ out of plane wagging within naphthalene ring \\
\hline $752 \mathrm{vw}$ & $\mathrm{CH}_{2}$ in plane rocking; $\mathrm{CH}_{3}$ in plane rocking \\
\hline $674 \mathrm{w}$ & In plane deformation within naphthalene \\
\hline $608 \mathrm{w}$ & In plane deformation within naphthalene ring \\
\hline $584 \mathrm{w}$ & In plane deformation within naphthalene ring and out of plane deformation indole ring \\
\hline $559 \mathrm{w}$ & Out of plane deformation of the indole ring \\
\hline $504 \mathrm{w}$ & In plane deformation within naphthalene ring \\
\hline $468 \mathrm{vw}$ & Out of plane deformation of the naphthalene ring \\
\hline $408 \mathrm{w}$ & $\mathrm{CH}$ out of plane wagging in naphthalene ring; Indole ring in plane deformation \\
\hline
\end{tabular}

Table 9: Vibrational assignment of peaks in the SERS spectrum of JWH-030. 


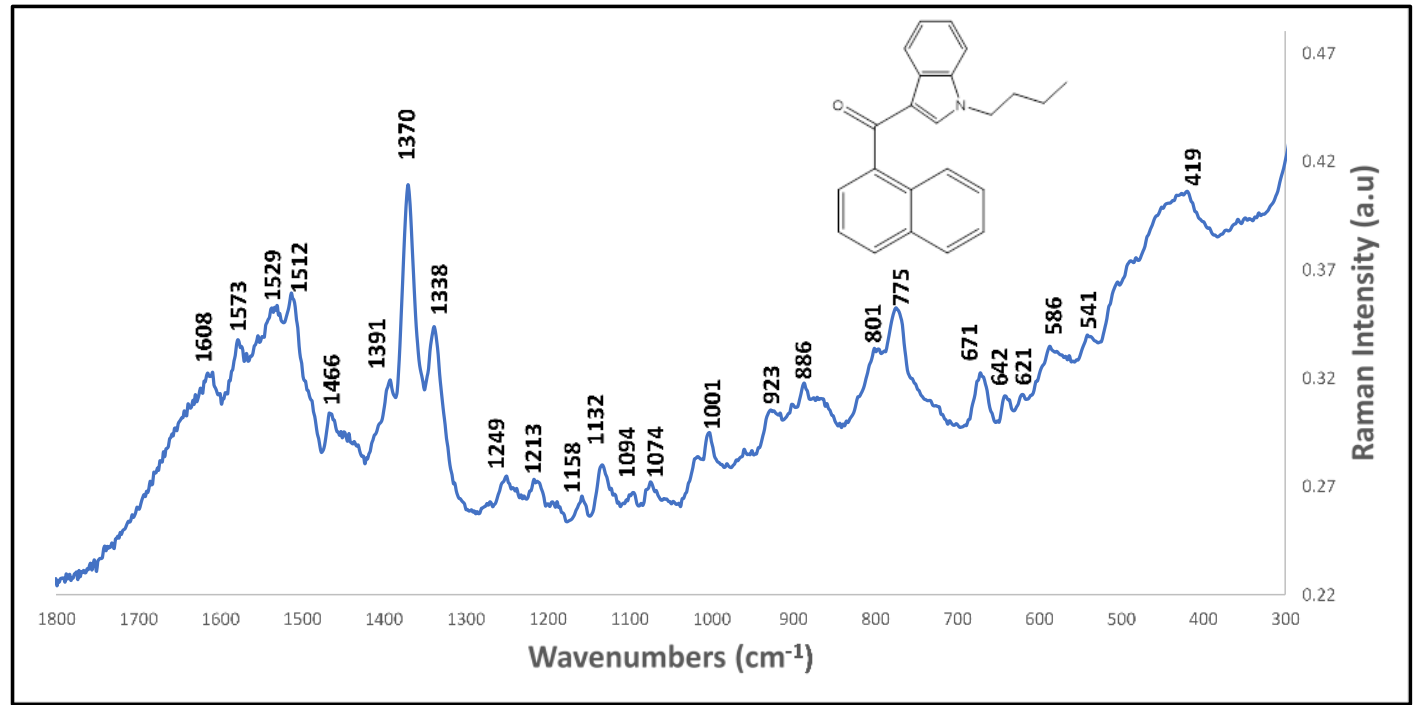

Figure 30: Chemical structure and SERS spectrum of JWH-073.

\begin{tabular}{|c|l|}
\hline Wavenumber & Assignment \\
\hline $1608 \mathrm{w}$ & $\mathrm{C}=\mathrm{C}$ stretching idole rings \\
$1573 \mathrm{w}$ & $\mathrm{C}=\mathrm{C}$ stretching naphthalene ring \\
$1529 \mathrm{w}$ & $\mathrm{C}=\mathrm{C}$ ) CCN stretching; $(\mathrm{CH}) \mathrm{NCH}_{2}$ in plane deformation \\
$1512 \mathrm{w}$ & $\mathrm{C}=\mathrm{C}$ ) $\mathrm{CCN}$ stretching; $\mathrm{CH}) \mathrm{NCH}_{2}$ in plane deformation \\
$1466 \mathrm{w}$ & $\mathrm{CH}$ scissoring; $\mathrm{CH}_{3}$ scissoring within idole ring; $\mathrm{CH}$ scissoring within naphthalene \\
& ring \\
$1391 \mathrm{vw}$ & $\mathrm{CH}$ in plane rocking within naphthalene ring \\
$1370 \mathrm{~s}$ & $\mathrm{C}=\mathrm{C}$ stretching in naphthalene ring \\
$1338 \mathrm{w}$ & $\mathrm{C}=\mathrm{C}$ stretching within indole ring; $\mathrm{CH}$ in plane scissoring within indole or \\
& naphthalene ring \\
$1249 \mathrm{w}$ & $\mathrm{HCH}$ out of plane wagging form tail; $\mathrm{CH}$ in plane scissoring within the idole or \\
& naphthalene ring \\
$1213 \mathrm{w}$ & $\mathrm{CH}$ in plane scissoring in naphthalene ring; $\mathrm{CH} \mathrm{H}_{2}$ out of plane twisting, $\mathrm{CH}_{3}$ out of \\
& plane twisting in the tail \\
$1158 \mathrm{w}$ & $\mathrm{HCCH}$ in plane stretching on the indole and naphthalene ring \\
$1132 \mathrm{vw}$ & $\mathrm{HCCH}$ in plane scissoring within the indole ring; $(\mathrm{CH}) \mathrm{NCH}$ in plane scissoring \\
$1074 \mathrm{vw}$ & $\mathrm{HCCCH}$ in plane scissoring of naphthalene ring \\
$1001 \mathrm{w}$ & Indole ring breathing; $\mathrm{CC}$ stretching within the tail \\
$923 \mathrm{vw}$ & $\mathrm{CH}$ out of plane wagging within the naphthalene ring \\
$886 \mathrm{w}$ & $\mathrm{CCH}$ stretching; in plane deformation within the idole ring or naphthalene ring \\
$801 \mathrm{vw}$ & $\mathrm{CH}$ in plane rocking; $\mathrm{CH}$ in plane rocking; $(\mathrm{CH}) \mathrm{NCH}$ out of plane wagging \\
$775 \mathrm{~m}$ & Indole ring breathing; $\mathrm{CH}$ out of plane wagging within naphthalene ring \\
$671 \mathrm{~m}$ & In plane deformation within naphthalene or indole ring \\
$642 \mathrm{w}$ & In plane deformation within naphthalene ring \\
$621 \mathrm{vw}$ & In plane deformation within naphthalene ring \\
$586 \mathrm{w}$ & In plane deformation within naphthalene ring and out of plane deformation indole \\
& ring \\
$541 \mathrm{vw}$ & In plane deformation within naphthalene or indole ring \\
$419 \mathrm{~m}$ & $\mathrm{CH}$ out of plane wagging in naphthalene ring; Indole ring in plane deformation \\
\hline
\end{tabular}

Table 10: Vibrational assignment of peaks in the SERS spectrum of JWH-073. 


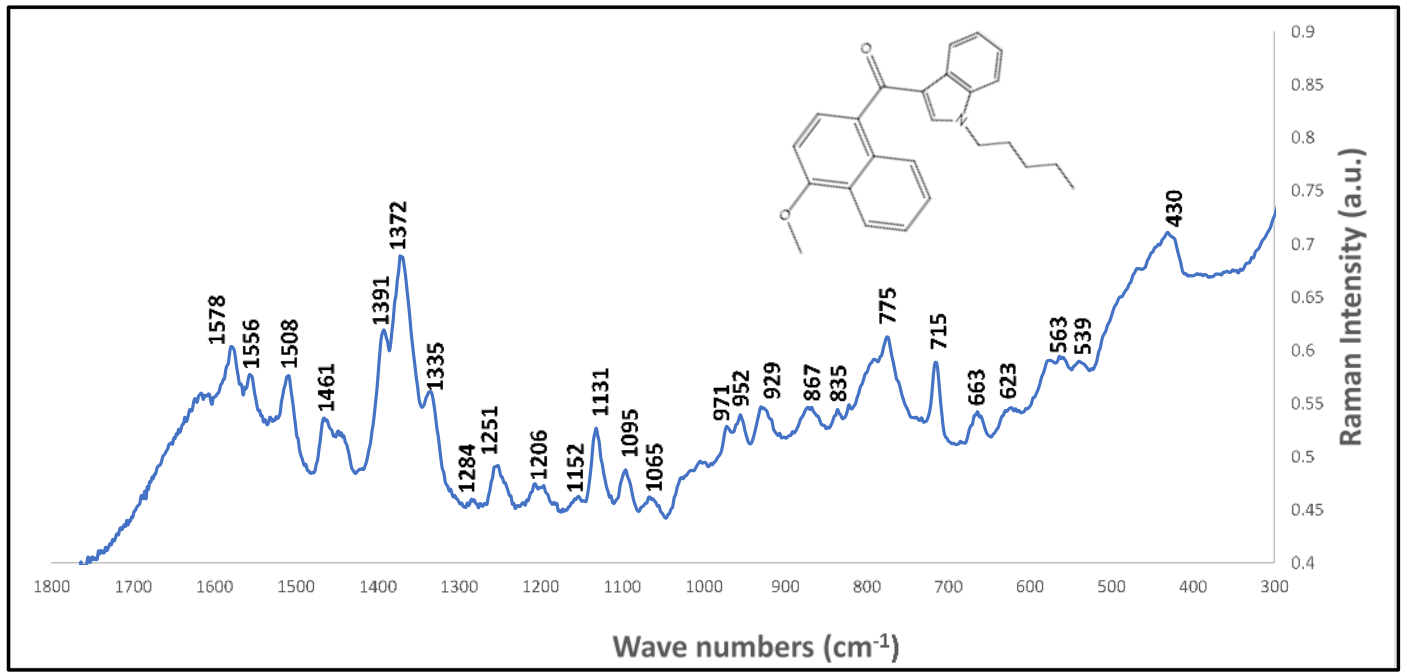

Figure 31: Chemical structure and SERS spectrum of JWH-081.

\begin{tabular}{|c|l|}
\hline Wavenumber & Assignment \\
\hline $1578 \mathrm{~m}$ & $\mathrm{C}=\mathrm{C}$ stretching naphthalene ring \\
$1556 \mathrm{w}$ & $\mathrm{C}=\mathrm{C}$ stretching indole and naphthalene ring \\
$1508 \mathrm{~m}$ & $\mathrm{C}=\mathrm{C}$ ) CCN stretching; $(\mathrm{CH}) \mathrm{NCH}_{2}$ in plane deformation \\
$1461 \mathrm{w}$ & $\mathrm{CH} \mathrm{C}_{2}$ scissoring; $\mathrm{CH}_{3}$ scissoring within idole ring; $\mathrm{CH}$ scissoring within naphthalene \\
& ring \\
$1391 \mathrm{vw}$ & $\mathrm{CH}$ in plane rocking within naphthalene ring \\
$1372 \mathrm{~s}$ & $\mathrm{C}=\mathrm{C}$ stretching in naphthalene ring \\
$1335 \mathrm{vw}$ & $\mathrm{C}=\mathrm{C}$ stretching within indole ring; $\mathrm{CH}$ in plane scissoring within indole or \\
& naphthalene ring \\
$1284 \mathrm{vw}$ & $\mathrm{CH}_{2}$ out of plane twisting; $\mathrm{CH}$ in plane scissoring for indole and naphthalene ring \\
$1251 \mathrm{~m}$ & $\mathrm{HCH}$ out of plane wagging form tail; $\mathrm{CH}$ in plane scissoring within the idole or \\
& naphthalene ring \\
$1206 \mathrm{w}$ & $\mathrm{CH}$ in plane scissoring in naphthalene ring; $\mathrm{CH} \mathrm{H}_{2}$ out of plane twisting, $\mathrm{CH}_{3}$ out of \\
& plane twisting in the tail \\
$1131 \mathrm{~m}$ & $\mathrm{HCCH}$ in plane scissoring within the indole ring; $(\mathrm{CH}) \mathrm{NCH}$ in plane scissoring \\
$1095 \mathrm{~m}$ & $\mathrm{HCCH}$ in plane scissoring within naphthalene ring; $\mathrm{CH}_{2}$ out of plane twisting, $\mathrm{CH}_{3}$ \\
& out of plane wagging on the tail \\
$971 \mathrm{vw}$ & $\mathrm{CH}$ out of plane wagging in naphthalene ring \\
$952 \mathrm{w}$ & $\mathrm{CH}$ out of plane wagging within the naphthalene ring \\
$929 \mathrm{w}$ & $\mathrm{CH}$ out of plane wagging within the naphthalene ring \\
$867 \mathrm{w}$ & $\mathrm{CCH}$ stretching; in plane deformation within the idole ring or naphthalene ring \\
$835 \mathrm{vw}$ & $\mathrm{CH}$ in plane rocking; $\mathrm{CH}$ in plane rocking; $(\mathrm{CH}) \mathrm{NCH}$ out of plane wagging \\
$775 \mathrm{~m}$ & Indole ring breathing; $\mathrm{CH}$ out of plane wagging within naphthalene ring \\
$715 \mathrm{~m}$ & $\mathrm{CH}$ out of plane wagging in indole and naphthalene ring \\
$663 \mathrm{w}$ & In plane deformation within naphthalene \\
$623 \mathrm{w}$ & Indole ring in plane deformation \\
$563 \mathrm{vw}$ & Indole ring out of plane deformation \\
$539 \mathrm{vw}$ & In plane deformation within naphthalene or indole ring \\
$430 \mathrm{~m}$ & $\mathrm{CH}$ out of plane wagging in naphthalene ring; Indole ring in plane deformation \\
\hline
\end{tabular}

Table 11: Vibrational assignment of peaks in the SERS spectrum of JWH-081. 


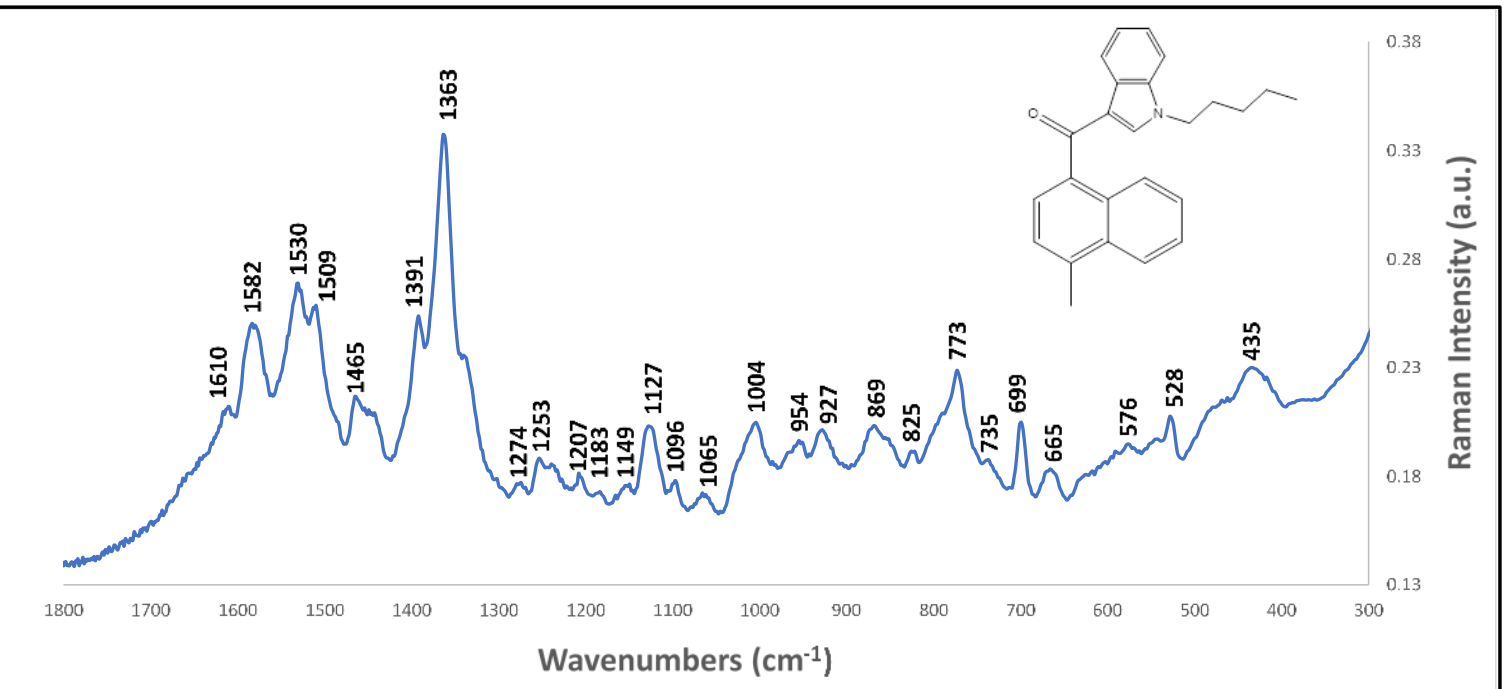

Figure 32: Chemical structure and SERS spectrum of JWH-122.

\begin{tabular}{|c|l|}
\hline Wavenumber & Assignment \\
\hline $1610 \mathrm{vw}$ & $\mathrm{C}=\mathrm{C}$ stretching idole rings \\
$1582 \mathrm{~m}$ & $\mathrm{C}=\mathrm{C}$ stretching naphthalene ring \\
$1530 \mathrm{~m}$ & $\mathrm{C}=\mathrm{C}) \mathrm{CCN}$ stretching; $(\mathrm{CH}) \mathrm{NCH}_{2}$ in plane deformation \\
$1509 \mathrm{w}$ & $\mathrm{C}=\mathrm{C}$ ) CCN stretching; $(\mathrm{CH}) \mathrm{NCH}_{2}$ in plane deformation \\
$1465 \mathrm{~m}$ & $\mathrm{CH}_{2}$ scissoring; $\mathrm{CH}_{3}$ scissoring within idole ring; $\mathrm{CH}$ scissoring in naphthalene ring \\
$1391 \mathrm{w}$ & $\mathrm{CH}$ in plane rocking within naphthalene ring \\
$1363 \mathrm{~s}$ & $\mathrm{C}=\mathrm{C}$ stretching in naphthalene ring \\
$1274 \mathrm{vw}$ & $\mathrm{CH}_{2}$ out of plane twisting; $\mathrm{CH}$ in plane scissoring for indole and naphthalene ring \\
$1253 \mathrm{vw}$ & $\mathrm{HCH}$ out of plane wagging form tail; $\mathrm{CH}$ in plane scissoring within the idole or \\
& naphthalene ring \\
$1207 \mathrm{vw}$ & $\mathrm{CH}$ in plane scissoring in naphthalene ring; $\mathrm{CH}_{2}$ out of plane twisting, $\mathrm{CH}_{3}$ out of \\
& plane twisting in the tail \\
$1183 \mathrm{vw}$ & $\mathrm{CH}$ in plane scissoring on indole ring; $\mathrm{CH}$ in plane rocking on indole and naphthalene \\
& ring \\
$1149 \mathrm{vw}$ & $\mathrm{HCCH}$ in plane stretching on the indole and naphthalene ring \\
$1127 \mathrm{~m}$ & $\mathrm{HCCH}$ in plane scissoring within the indole ring; $(\mathrm{CH})$ NCH in plane scissoring \\
$1065 \mathrm{vw}$ & $\mathrm{HCCCH}$ in plane scissoring of naphthalene ring \\
$1004 \mathrm{w}$ & Indole ring breathing; $\mathrm{CC}$ stretching within the tail \\
$954 \mathrm{w}$ & $\mathrm{CH}$ out of plane wagging within the naphthalene ring \\
$927 \mathrm{w}$ & $\mathrm{CH}$ out of plane wagging within the naphthalene ring \\
$869 \mathrm{w}$ & $\mathrm{CCH}$ stretching; in plane deformation within the idole ring or naphthalene ring \\
$825 \mathrm{vw}$ & $\mathrm{CH} 2$ in plane rocking; $\mathrm{CH}$ in plane rocking; $(\mathrm{CH}) \mathrm{NCH}$ out of plane wagging \\
$773 \mathrm{~m}$ & Indole ring breathing; $\mathrm{CH}$ out of plane wagging within naphthalene ring \\
$735 \mathrm{vw}$ & $\mathrm{CH}$ indole and naphthalene ring out of plane wagging \\
$699 \mathrm{~m}$ & $\mathrm{CH}$ indole and naphthalene ring out of plane wagging \\
$665 \mathrm{w}$ & In plane deformation within naphthalene \\
$576 \mathrm{vw}$ & In plane deformation within naphthalene ring and out of plane deformation indole \\
$528 \mathrm{w}$ & ring \\
$435 \mathrm{w}$ & $\mathrm{In}$ plane deformation within naphthalene or indole ring \\
$\mathrm{CH}$ out of plane wagging in naphthalene ring; Indole ring in plane deformation
\end{tabular}

Table 12: Vibrational assignment of peaks in the SERS spectrum of JWH-122. 


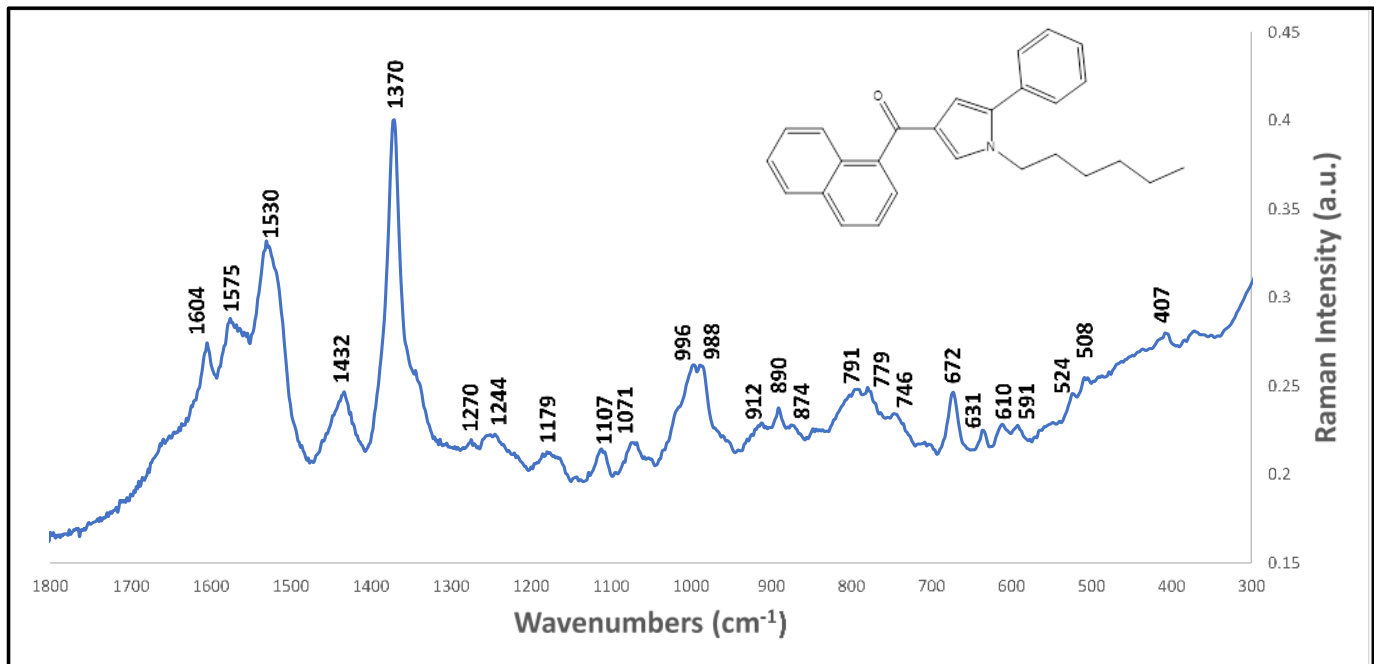

Figure 33: Chemical structure and SERS spectrum of JWH-147.

\begin{tabular}{|c|c|}
\hline Wavenumber & Assignment \\
\hline $1604 \mathrm{w}$ & $\mathrm{C}=\mathrm{C}$ stretching idole rings \\
\hline $1575 \mathrm{w}$ & $\mathrm{C}=\mathrm{C}$ stretching naphthalene ring \\
\hline $1530 \mathrm{~m}$ & $(\mathrm{C}=\mathrm{C}) \mathrm{CCN}$ stretching; $(\mathrm{CH}) \mathrm{NCH} 2$ out of plane deformation \\
\hline $1432 \mathrm{~m}$ & $\mathrm{CH}$ out of plane rocking in the naphthalene ring \\
\hline $1370 \mathrm{~m}$ & $\mathrm{C}=\mathrm{C}$ stretching in naphthalene ring \\
\hline $1270 \mathrm{vw}$ & $\mathrm{CH}_{2}$ out of plane twisting; $\mathrm{CH}$ in plane scissoring for indole and naphthalene ring \\
\hline $1244 \mathrm{vw}$ & $\begin{array}{l}\mathrm{HCH} \text { out of plane wagging form tail; } \mathrm{CH} \text { in plane scissoring within the idole or } \\
\text { naphthalene ring }\end{array}$ \\
\hline $1179 \mathrm{vw}$ & $\mathrm{HCCCH}$ in plane scissoring of naphthalene ring \\
\hline $1107 \mathrm{w}$ & $\begin{array}{l}(\mathrm{HCCH}) \text { in plane scissoring of naphthalene ring; } \mathrm{CH}_{2} \text { out of plane twisting, } \mathrm{CH}_{3} \text { out of } \\
\text { plane wagging from the tail }\end{array}$ \\
\hline $1071 \mathrm{w}$ & $\mathrm{HCCCH}$ in plane scissoring of naphthalene ring \\
\hline $996 \mathrm{vw}$ & $\mathrm{C}-\mathrm{C}$ tail stretching; indole and naphthalene ring stretching \\
\hline $988 \mathrm{vW}$ & $\mathrm{CH}$ naphthalene ring out of plane wagging \\
\hline $890 \mathrm{vw}$ & $\mathrm{CCH}_{3}$ stretching; indole and naphthalene ring stretching \\
\hline $874 \mathrm{w}$ & $\mathrm{CCH}_{3}$ stretching; in plane deformation within the idole ring or naphthalene ring \\
\hline $791 \mathrm{vw}$ & $\mathrm{CH}_{2}$ in plane rocking; $\mathrm{CH}_{3}$ in plane rocking; $(\mathrm{CH}) \mathrm{NCH}$ out of plane wagging \\
\hline $779 \mathrm{w}$ & Indole ring breathing; $\mathrm{CH}$ out of plane wagging within naphthalene ring \\
\hline $746 \mathrm{w}$ & $\mathrm{CH}_{2}$ in plane rocking; $\mathrm{CH}_{3}$ in plane rocking \\
\hline $672 \mathrm{~m}$ & In plane deformation within naphthalene \\
\hline $631 \mathrm{vw}$ & In plane deformation within naphthalene or indole ring \\
\hline $610 \mathrm{vw}$ & In plane deformation within naphthalene ring \\
\hline $591 \mathrm{vw}$ & In plane deformation within naphthalene ring and out of plane deformation indole ring \\
\hline $524 \mathrm{vw}$ & In plane deformation within naphthalene or indole ring \\
\hline $508 \mathrm{vw}$ & In plane deformation within naphthalene ring \\
\hline $407 \mathrm{vw}$ & $\mathrm{CH}$ out of plane wagging in naphthalene ring; Indole ring in plane deformation \\
\hline
\end{tabular}

Table 13: Vibrational assignment of peaks in the SERS spectrum of JWH-147. 


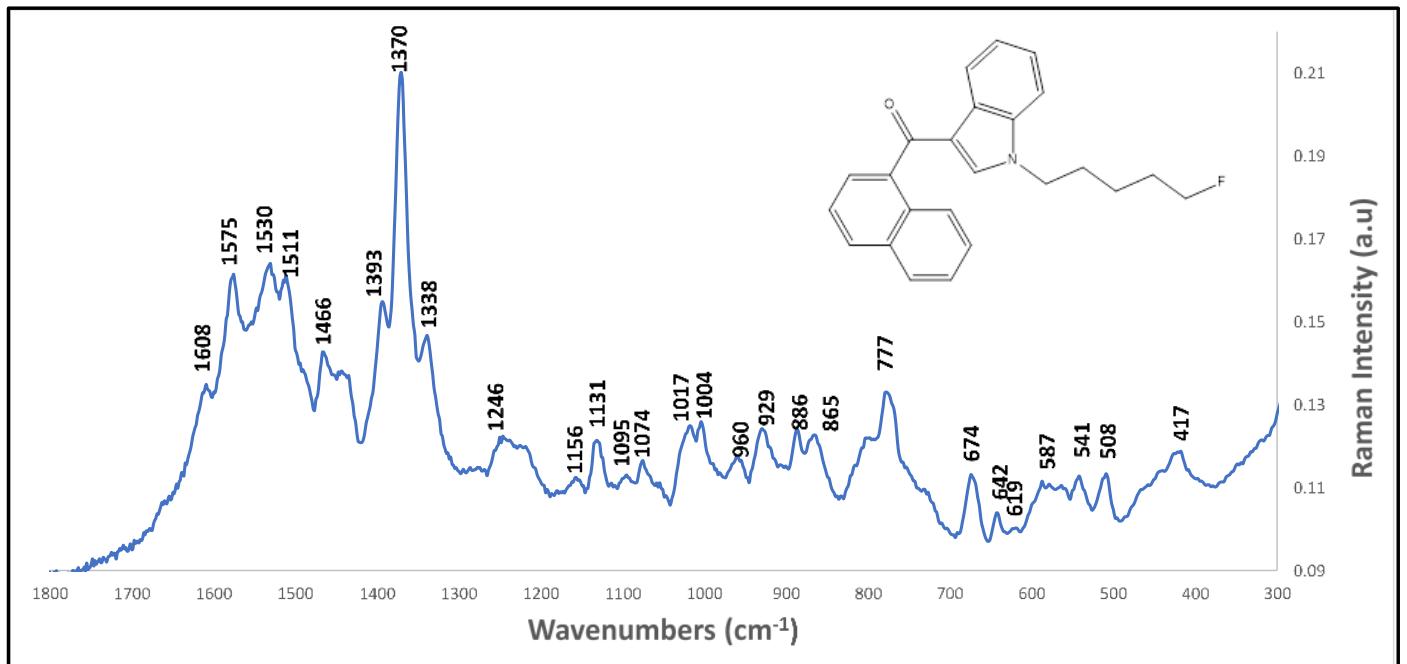

Figure 34:Chemical structure and SERS spectrum of AM-2201.

\begin{tabular}{|c|c|}
\hline Wavenumber & Assignment \\
\hline $1608 \mathrm{vw}$ & $\mathrm{C}=\mathrm{C}$ stretching idole rings \\
\hline $1575 \mathrm{~m}$ & $\mathrm{C}=\mathrm{C}$ stretching naphthalene ring \\
\hline $1530 \mathrm{~m}$ & $(\mathrm{C}=\mathrm{C}) \mathrm{CCN}$ stretching; $(\mathrm{CH}) \mathrm{NCH} 2$ out of plane deformation \\
\hline $1511 \mathrm{~m}$ & $(\mathrm{C}=\mathrm{C}) \mathrm{CCN}$ stretching; $(\mathrm{CH}) \mathrm{NCH}_{2}$ in plane deformation \\
\hline $1466 \mathrm{~m}$ & $\mathrm{CH}_{2}$ scissoring; $\mathrm{CH}_{3}$ scissoring within idole ring; $\mathrm{CH}$ scissoring in naphthalene ring \\
\hline $1393 \mathrm{w}$ & $\mathrm{CH}$ in plane rocking within naphthalene ring \\
\hline $1370 \mathrm{~s}$ & $\mathrm{C}=\mathrm{C}$ stretching in naphthalene ring \\
\hline $1338 \mathrm{w}$ & $\mathrm{C}=\mathrm{C}$ stretching within indole ring; $\mathrm{CH}$ in plane scissoring in indole or naphthalene ring \\
\hline $1246 \mathrm{~m}$ & $\begin{array}{l}\mathrm{HCH} \text { out of plane wagging form tail; } \mathrm{CH} \text { in plane scissoring within the idole or naphthalene } \\
\text { ring }\end{array}$ \\
\hline $1156 \mathrm{vw}$ & $\mathrm{HCCH}$ in plane scissoring within the indole or naphthalene ring \\
\hline $1131 \mathrm{~m}$ & $\mathrm{HCCH}$ in plane scissoring within the indole ring; $(\mathrm{CH}) \mathrm{NCH}$ in plane scissoring \\
\hline $1095 \mathrm{vw}$ & $\begin{array}{l}(\mathrm{HCCH}) \text { in plane scissoring of naphthalene ring; } \mathrm{CH}_{2} \text { out of plane twisting, } \mathrm{CH}_{3} \text { out of plane } \\
\text { wagging from the tail }\end{array}$ \\
\hline $1074 \mathrm{w}$ & $\mathrm{HCCCH}$ in plane scissoring of naphthalene ring \\
\hline $1004 \mathrm{w}$ & Indole ring breathing; $\mathrm{CC}$ stretching within the tail \\
\hline $960 \mathrm{w}$ & $\mathrm{CH}$ out of plane wagging within the naphthalene ring \\
\hline $929 \mathrm{~m}$ & $\mathrm{CH}$ out of plane wagging within the naphthalene ring \\
\hline $886 \mathrm{w}$ & $\mathrm{CCH}_{3}$ stretching; in plane deformation within indole or naphthalene ring \\
\hline $865 \mathrm{w}$ & $\mathrm{CCH}_{3}$ stretching; in plane deformation within the idole ring or naphthalene ring; $\mathrm{CF}$ stretch \\
\hline $801 \mathrm{w}$ & $\mathrm{CH}_{2}$ in plane rocking; $\mathrm{CH}_{3}$ in plane rocking; $(\mathrm{CH}) \mathrm{NCH}$ out of plane wagging \\
\hline $777 \mathrm{~m}$ & Indole ring breathing; $\mathrm{CH}$ out of plane wagging within naphthalene ring \\
\hline $674 \mathrm{~m}$ & In plane deformation within naphthalene \\
\hline $642 \mathrm{w}$ & In plane deformation within naphthalene or indole ring \\
\hline $619 \mathrm{vw}$ & In plane deformation within naphthalene ring \\
\hline $587 \mathrm{vw}$ & In plane deformation within naphthalene ring and out of plane deformation indole ring \\
\hline $541 \mathrm{~m}$ & In plane deformation within naphthalene or indole ring \\
\hline $508 \mathrm{~m}$ & In plane deformation within naphthalene ring \\
\hline $417 \mathrm{~m}$ & $\mathrm{CH}$ out of plane wagging in naphthalene ring; Indole ring in plane deformation \\
\hline
\end{tabular}

Table 14: Vibrational assignment of peaks in the SERS spectrum of AM-2201. 


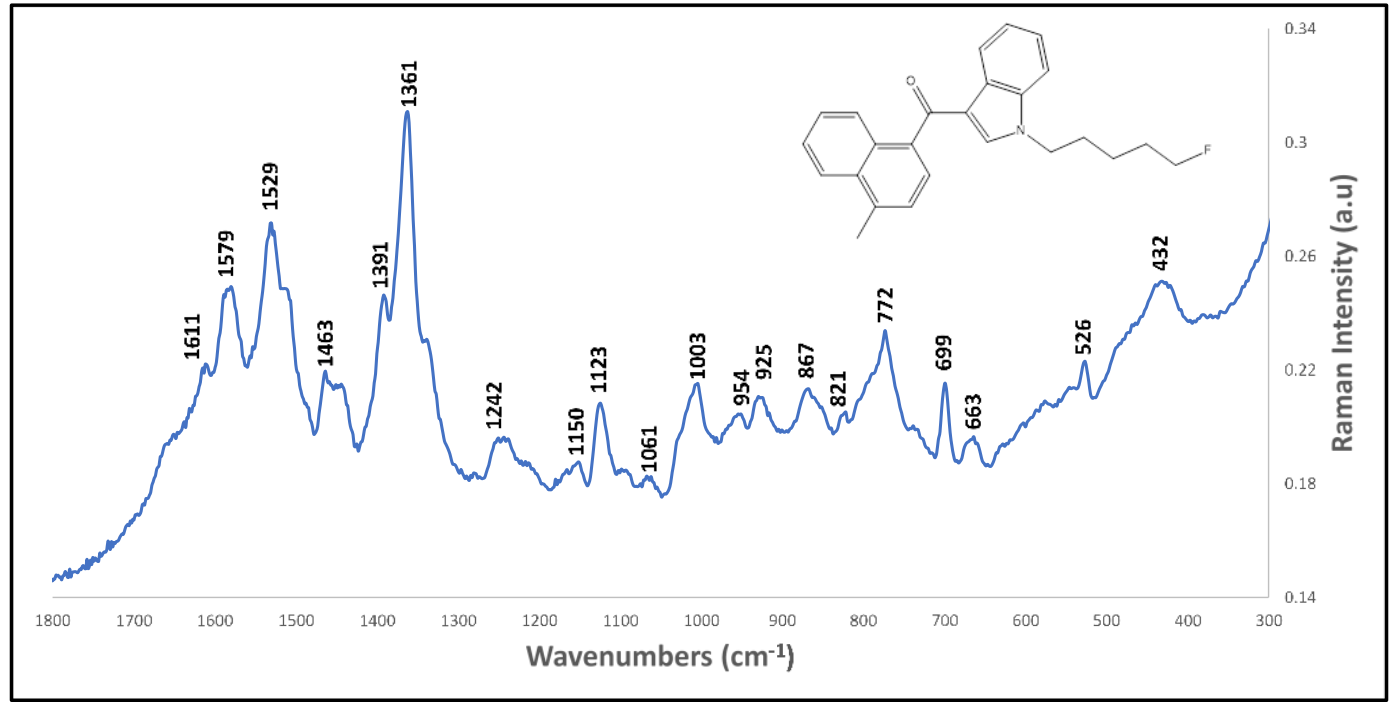

Figure 35: Chemical structure and SERS spectrum of MAM-2201.

\begin{tabular}{|l|l|}
\hline Wavenumber & Assignment \\
\hline $1611 \mathrm{vw}$ & $\mathrm{C}=\mathrm{C}$ stretching idole rings \\
$1579 \mathrm{~m}$ & $\mathrm{C}=\mathrm{C}$ stretching naphthalene ring \\
$1529 \mathrm{~s}$ & $\mathrm{C}=\mathrm{C}) \mathrm{CCN}$ stretching; $\mathrm{CH}) \mathrm{NCH}$ 2 out of plane deformation \\
$1463 \mathrm{~m}$ & $\mathrm{CH}_{2}$ scissoring; $\mathrm{CH}_{3}$ scissoring within idole ring; $\mathrm{CH}$ scissoring within naphthalene ring \\
$1391 \mathrm{vw}$ & $\mathrm{CH}$ in plane rocking within naphthalene ring \\
$1361 \mathrm{vs}$ & $\mathrm{C}=\mathrm{C}$ stretching within naphthalene ring \\
$1242 \mathrm{~m}$ & $\mathrm{HCH}$ out of plane wagging form tail; $\mathrm{CH}$ in plane scissoring within the idole or naphthalene \\
& ring \\
$1150 \mathrm{w}$ & $\mathrm{HCCH}$ in plane scissoring within the indole or naphthalene ring \\
$1123 \mathrm{~m}$ & $\mathrm{HCCH}$ in plane scissoring within the indole ring; $(\mathrm{CH})$ NCH in plane scissoring \\
$1003 \mathrm{~m}$ & Indole ring breathing; CC stretching within the tail \\
$954 \mathrm{w}$ & $\mathrm{CH}$ out of plane wagging within the naphthalene ring \\
$925 \mathrm{w}$ & $\mathrm{CH}$ out of plane wagging within the naphthalene ring \\
$867 \mathrm{w}$ & $\mathrm{CCH}$ stretching; in plane deformation within the idole ring or naphthalene ring; $\mathrm{CF}$ stretch \\
$821 \mathrm{vw}$ & $\mathrm{CH}$ in plane rocking; $\mathrm{CH}$ in plane rocking; $(\mathrm{CH}) \mathrm{NCH}$ out of plane wagging \\
$772 \mathrm{~m}$ & Indole ring breathing; $\mathrm{CH}$ out of plane wagging within naphthalene ring \\
$699 \mathrm{~m}$ & In plane deformation within naphthalene ring \\
$663 \mathrm{w}$ & In plane deformation within naphthalene ring \\
$526 \mathrm{w}$ & In plane deformation within naphthalene or indole ring \\
$432 \mathrm{~m}$ & $\mathrm{CH}$ out of plane wagging in naphthalene ring; Indole ring in plane deformation \\
\hline
\end{tabular}

Table 15: Vibrational assignment of peaks in the SERS spectrum of MAM-2201. 


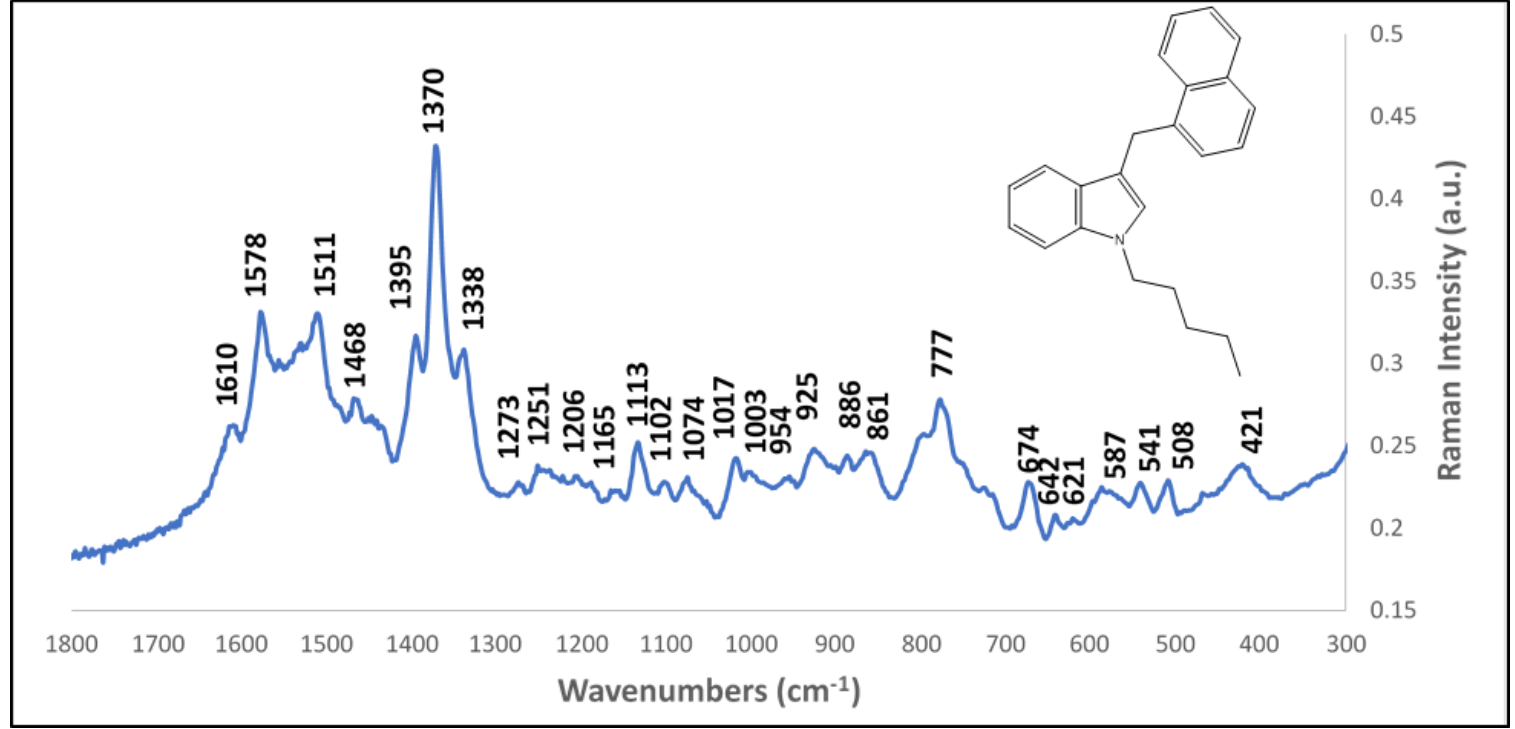

Figure 36: Chemical structure and SERS spectrum of JWH-175.

\begin{tabular}{|c|c|}
\hline Wavenumber & Assignment \\
\hline $1610 \mathrm{vw}$ & $\mathrm{C}=\mathrm{C}$ stretching idole rings \\
\hline $1578 \mathrm{~m}$ & $\mathrm{C}=\mathrm{C}$ stretching naphthalene ring \\
\hline $1511 \mathrm{~m}$ & $(\mathrm{C}=\mathrm{C}) \mathrm{CCN}$ stretching; $(\mathrm{CH}) \mathrm{NCH}_{2}$ in plane deformation \\
\hline $1468 \mathrm{vw}$ & $\mathrm{CH}_{2}$ scissoring; $\mathrm{CH}_{3}$ scissoring within idole ring; $\mathrm{CH}$ scissoring within naphthalene ring \\
\hline $1395 \mathrm{w}$ & $\mathrm{CH}$ in plane rocking within naphthalene ring \\
\hline $1371 \mathrm{~s}$ & $\mathrm{C}=\mathrm{C}$ stretching in naphthalene ring \\
\hline $1338 \mathrm{w}$ & $\mathrm{C}=\mathrm{C}$ stretching within indole ring; $\mathrm{CH}$ in plane scissoring within indole or naphthalene ring \\
\hline $1273 \mathrm{vw}$ & $\mathrm{CH}_{2}$ out of plane twisting; $\mathrm{CH}$ in plane scissoring for indole and naphthalene ring \\
\hline $1251 \mathrm{w}$ & $\begin{array}{l}\mathrm{HCH} \text { out of plane wagging form tail; } \mathrm{CH} \text { in plane scissoring within the idole or naphthalene } \\
\text { ring }\end{array}$ \\
\hline $1206 \mathrm{vw}$ & $\begin{array}{l}\mathrm{CH} \text { in plane scissoring in naphthalene ring; } \mathrm{CH}_{2} \text { out of plane twisting, } \mathrm{CH}_{3} \text { out of plane } \\
\text { twisting in the tail }\end{array}$ \\
\hline $1165 \mathrm{vw}$ & $\mathrm{HCCH}$ in plane scissoring within the indole or naphthalene ring \\
\hline $1133 \mathrm{~m}$ & $\mathrm{HCCH}$ in plane scissoring within the indole ring; $(\mathrm{CH}) \mathrm{NCH}$ in plane scissoring \\
\hline $1102 \mathrm{vw}$ & $\begin{array}{l}(\mathrm{HCCH}) \text { in plane scissoring of naphthalene ring; } \mathrm{CH}_{2} \text { out of plane twisting, } \mathrm{CH}_{3} \text { out of plane } \\
\text { wagging from the tail }\end{array}$ \\
\hline $1074 \mathrm{w}$ & $\mathrm{HCCCH}$ in plane scissoring of naphthalene ring \\
\hline $1017 \mathrm{w}$ & Indole ring breathing; $\mathrm{C}-\mathrm{C}$ stretching in the tail \\
\hline $1003 \mathrm{vw}$ & Indole ring breathing; $\mathrm{CC}$ stretching within the tail \\
\hline $954 \mathrm{vw}$ & $\mathrm{CH}$ out of plane wagging within the naphthalene ring \\
\hline $925 \mathrm{w}$ & $\mathrm{CH}$ out of plane wagging within the naphthalene ring \\
\hline $886 \mathrm{vw}$ & $\mathrm{CCH}_{3}$ stretching; in plane deformation within indole or naphthalene ring \\
\hline $861 \mathrm{w}$ & $\mathrm{CCH}_{3}$ stretching; in plane deformation within the idole ring or naphthalene ring \\
\hline $777 \mathrm{~m}$ & Indole ring breathing; $\mathrm{CH}$ out of plane wagging within naphthalene ring \\
\hline $674 \mathrm{~m}$ & In plane deformation within naphthalene \\
\hline $642 \mathrm{vw}$ & In plane deformation within naphthalene or indole ring \\
\hline $621 \mathrm{vw}$ & In plane deformation within naphthalene ring \\
\hline $587 \mathrm{w}$ & In plane deformation within naphthalene ring and out of plane deformation indole ring \\
\hline $541 \mathrm{w}$ & In plane deformation within naphthalene or indole ring \\
\hline $508 \mathrm{w}$ & In plane deformation within naphthalene ring \\
\hline $421 \mathrm{w}$ & $\mathrm{CH}$ out of plane wagging in naphthalene ring; Indole ring in plane deformation \\
\hline
\end{tabular}

Table 16: Vibrational assignment of peaks in the SERS spectrum of JWH-175. 


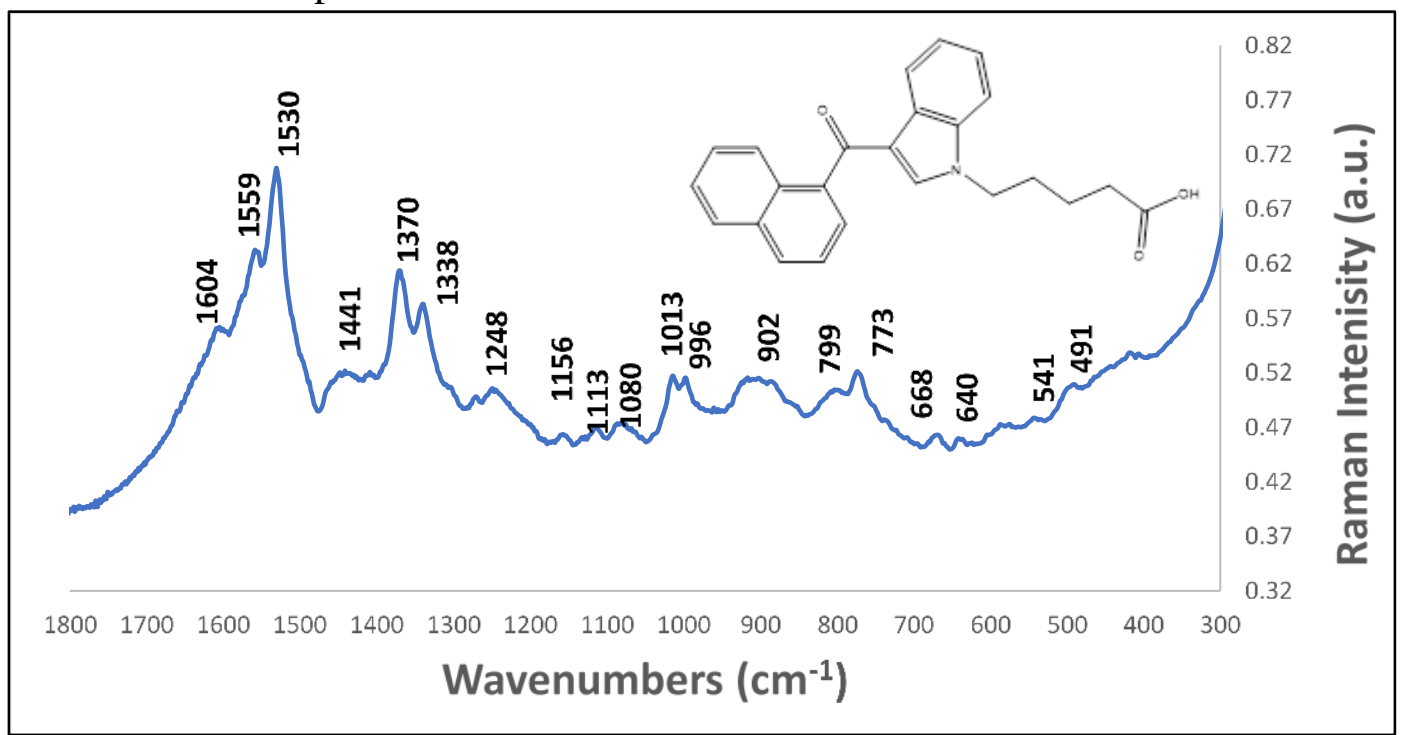

Figure 37: Chemical structure and SERS spectrum of JWH-018 N-pentanoic acid.

\begin{tabular}{|c|l|}
\hline Wavenumber & Assignment \\
\hline $1604 \mathrm{vw}$ & $\mathrm{C}=\mathrm{C}$ stretching idole rings \\
$1559 \mathrm{~m}$ & $\mathrm{C}=\mathrm{C}$ stretching naphthalene ring \\
$1530 \mathrm{~s}$ & $(\mathrm{C}=\mathrm{C}) \mathrm{CCN}$ stretching; $(\mathrm{CH}) \mathrm{NCH}_{2}$ in plane deformation \\
$1441 \mathrm{vw}$ & $\mathrm{CH}$ scissoring; $\mathrm{CH}_{3}$ scissoring within idole ring; $\mathrm{CH}$ scissoring within naphthalene ring \\
$1370 \mathrm{~m}$ & $\mathrm{C}=\mathrm{C}$ stretching in naphthalene ring \\
$1338 \mathrm{~m}$ & $\mathrm{C}=\mathrm{C}$ stretching within indole ring; $\mathrm{CH}$ in plane scissoring within indole or naphthalene ring \\
$1248 \mathrm{w}$ & $\mathrm{HCH}$ out of plane wagging form tail; $\mathrm{CH}$ in plane scissoring within the idole or naphthalene \\
& ring \\
$1156 \mathrm{vw}$ & $\mathrm{HCCH}$ in plane scissoring within the indole or naphthalene ring \\
$1113 \mathrm{vw}$ & $\mathrm{HCCH}$ in plane scissoring within the indole ring; $(\mathrm{CH}) \mathrm{NCH}$ in plane scissoring \\
$1080 \mathrm{vw}$ & $\mathrm{HCCCH}$ in plane scissoring of naphthalene ring \\
$1013 \mathrm{w}$ & Indole ring breathing; $\mathrm{C}-\mathrm{C}$ stretching in the tail \\
$996 \mathrm{vw}$ & $\mathrm{CH}$ out of plane wagging within the naphthalene ring \\
$902 \mathrm{w}$ & $\mathrm{CH}$ out of plane wagging within the naphthalene ring \\
$799 \mathrm{vw}$ & Indole ring breathing; $\mathrm{CH}$ out of plane wagging within naphthalene ring \\
$773 \mathrm{w}$ & Indole ring breathing; $\mathrm{CH}$ out of plane wagging within naphthalene ring \\
$668 \mathrm{vw}$ & In plane deformation within naphthalene \\
$640 \mathrm{vw}$ & In plane deformation within naphthalene or indole ring \\
$541 \mathrm{vw}$ & In plane deformation within naphthalene or indole ring \\
$491 \mathrm{vw}$ & CH out of plane wagging in naphthalene ring; Indole ring in plane deformation \\
\hline
\end{tabular}

Table 17: Vibrational assignment of peaks in the SERS spectrum of JWH-018 Npentanoic acid. 


\subsubsection{JWH-018 N-(5-hydroxypentyl) metabolite}

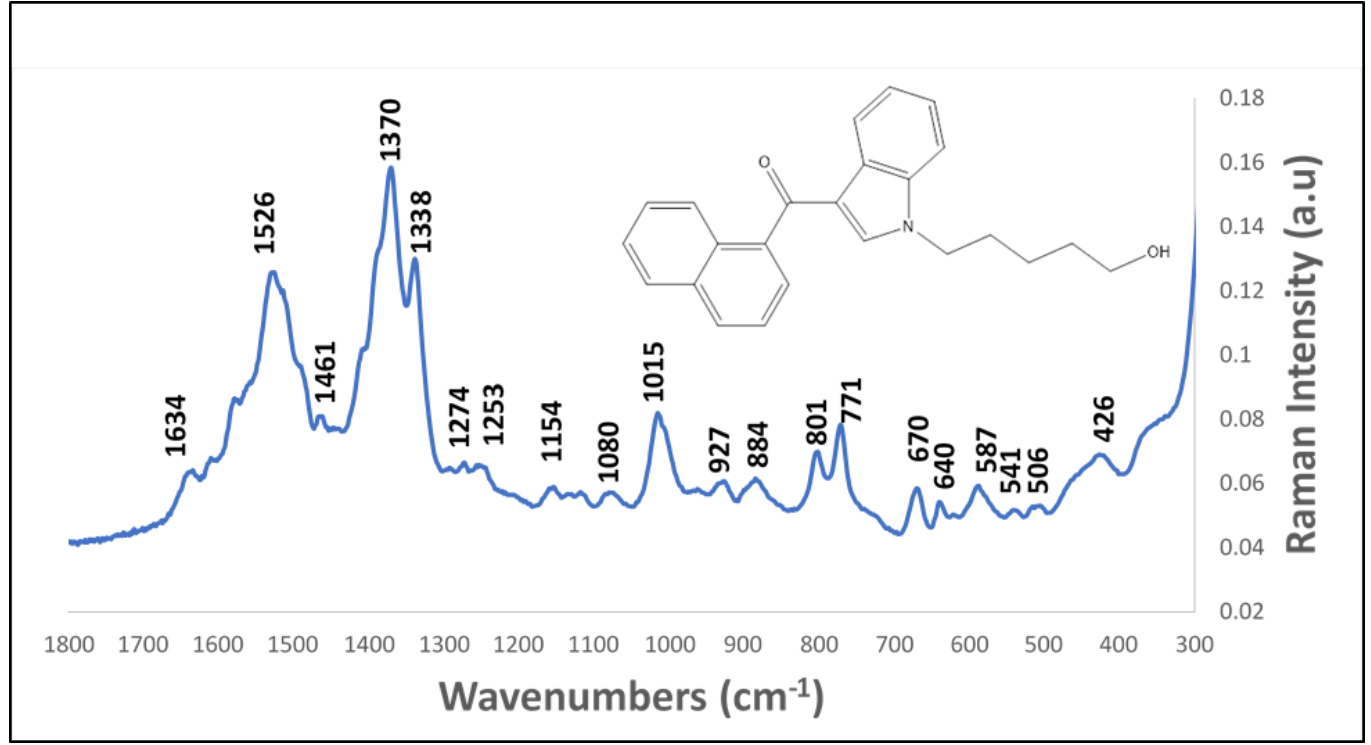

Figure 38: Chemical structure and SERS spectrum of JWH-018 N-(5-hydroxypentyl) metabolite.

\begin{tabular}{|c|l|}
\hline Wavenumber & Assignment \\
\hline $1634 \mathrm{vw}$ & $\mathrm{C}=\mathrm{C}$ stretching idole rings \\
$1526 \mathrm{~m}$ & $\mathrm{C}=\mathrm{C}$ stretching naphthalene ring \\
$1461 \mathrm{vw}$ & $\mathrm{CH}_{2}$ scissoring; $\mathrm{CH}_{3}$ scissoring within idole ring; CH scissoring within naphthalene ring \\
$1370 \mathrm{~s}$ & $\mathrm{C}=\mathrm{C}$ stretching in naphthalene ring \\
$1338 \mathrm{~m}$ & $\mathrm{C}=\mathrm{C}$ stretching within indole ring; $\mathrm{CH}$ in plane scissoring within indole or naphthalene ring \\
$1274 \mathrm{vw}$ & $\mathrm{CH}$ out of plane twisting; $\mathrm{CH}$ in plane scissoring for indole and naphthalene ring \\
$1253 \mathrm{vw}$ & $\mathrm{HCH}$ out of plane wagging form tail; $\mathrm{CH}$ in plane scissoring within the idole or naphthalene \\
& ring \\
$1154 \mathrm{vw}$ & $\mathrm{HCCH}$ in plane scissoring within the indole or naphthalene ring \\
$1080 \mathrm{vw}$ & $\mathrm{HCCCH}$ in plane scissoring of naphthalene ring \\
$1015 \mathrm{~m}$ & Indole ring breathing; C-C stretching in the tail \\
$1003 \mathrm{vw}$ & Indole ring breathing; CC stretching within the tail \\
$927 \mathrm{w}$ & $\mathrm{CH}$ out of plane wagging within the naphthalene ring \\
$884 \mathrm{vw}$ & $\mathrm{CCH}$ stretching; in plane deformation within indole or naphthalene ring \\
$801 \mathrm{w}$ & $\mathrm{CCH}$ stretching; in plane deformation within the idole ring or naphthalene ring \\
$771 \mathrm{~m}$ & Indole ring breathing; CH out of plane wagging within naphthalene ring \\
$670 \mathrm{~m}$ & In plane deformation within naphthalene \\
$587 \mathrm{w}$ & In plane deformation within naphthalene ring and out of plane deformation indole ring \\
$541 \mathrm{w}$ & In plane deformation within naphthalene or indole ring \\
$506 \mathrm{w}$ & In plane deformation within naphthalene ring \\
$426 \mathrm{w}$ & $\mathrm{CH}$ out of plane wagging in naphthalene ring; Indole ring in plane deformation \\
\hline
\end{tabular}

Table 18: Vibrational assignment of peaks in the SERS spectrum of JWH-018 N-(5hydroxypentyl) metabolite. 
6.3.11 JWH-018 N-pentanoic acid metabolite pure drug and extraction comparison

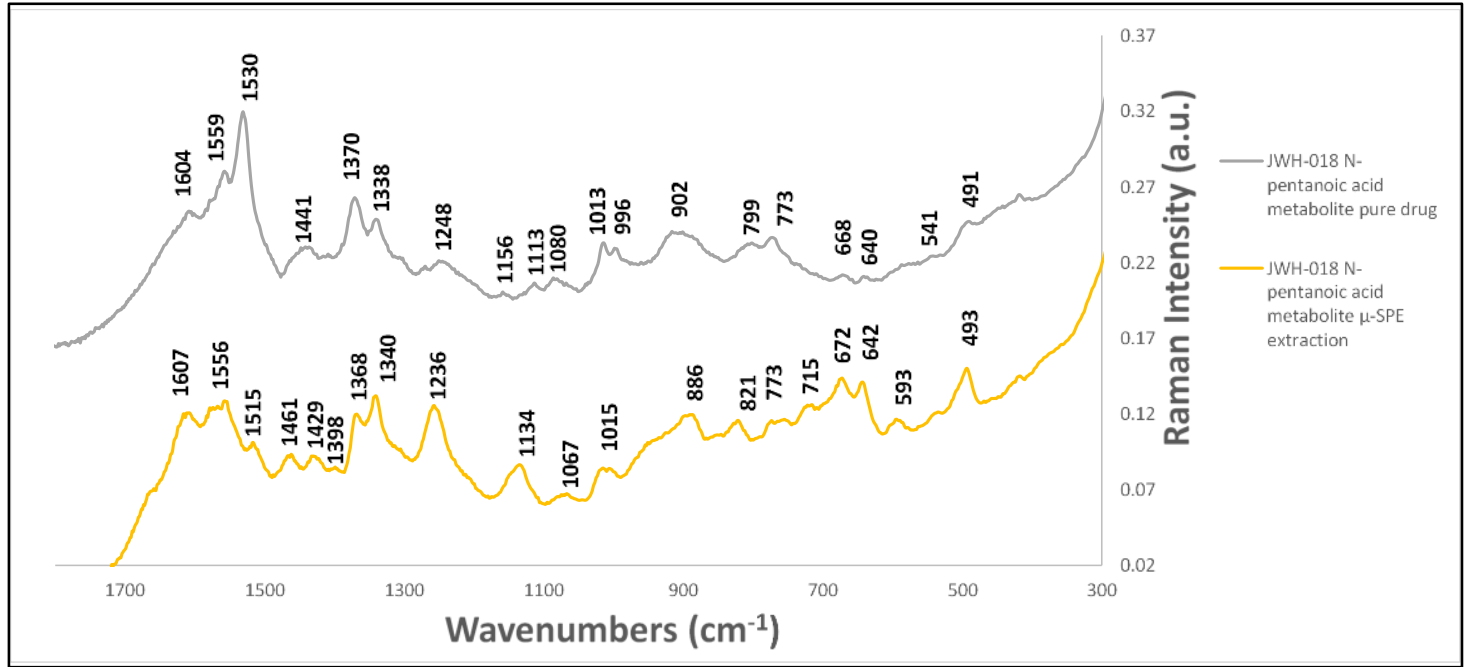

Figure 39:A comparison of the SERS spectrum of JWH-018 N-pentanoic acid metabolite as the pure drug and in a spiked urine sample after $\mu$-SPE.

6.3.12 JWH-018 N-(5-hydroxypentyl) metabolite pure drug and extraction comparison

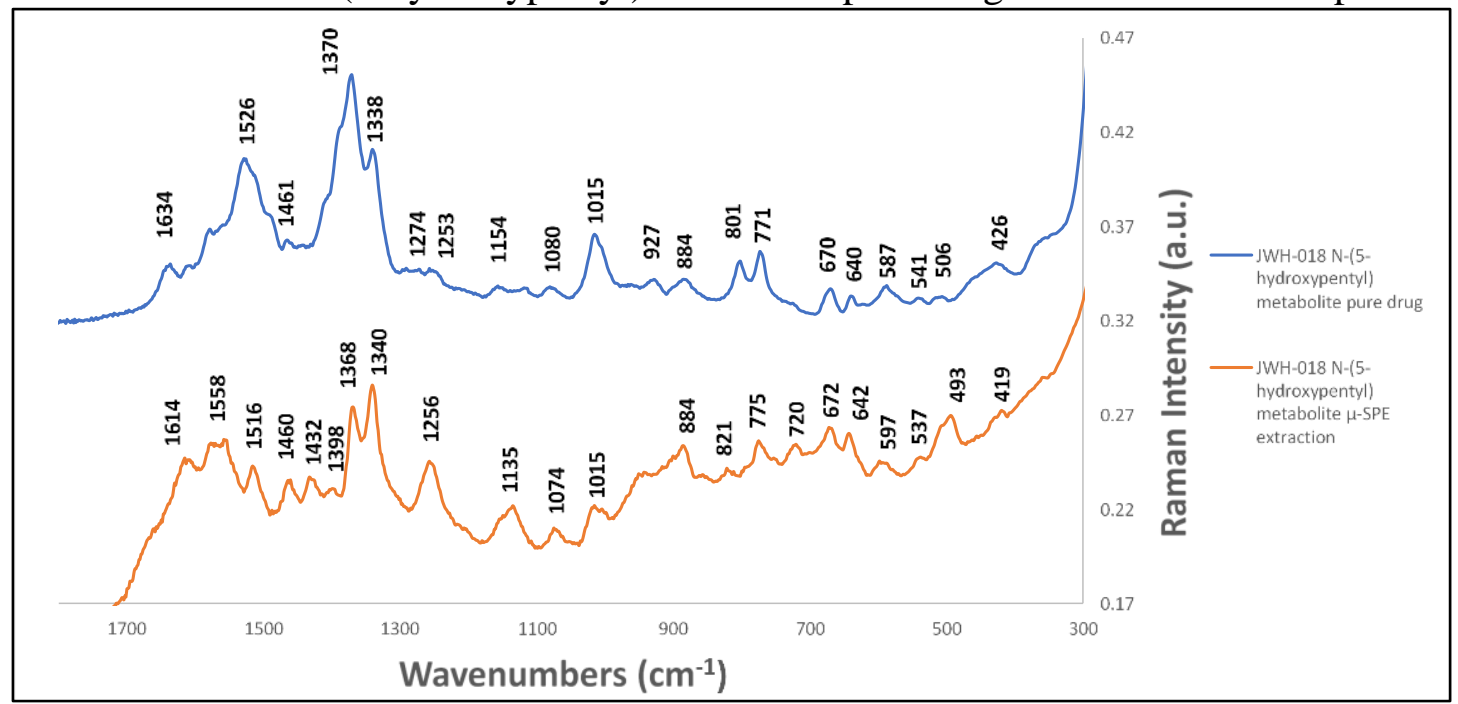

Figure 40: A comparison of the SERS spectrum of JWH-018 N-(5-hydroxypentyl) metabolite as the pure drug and in a spiked urine sample after $\mu$-SPE.

\section{CONCLUSIONS AND FUTURE WORK}

This work shows the development of a rapid screening technique for the detection of trace levels of synthetic cannabinoid parent compounds and two JWH-018 metabolites. Initially, four synthetic cannabinoid parent compounds (JWH-018, JWH-0073, JWH-081 and JWH-122) were analyzed via previously developed SERS method for the detection of 
trace levels of benzodiazepines. To the author's knowledge, this was the first paper published on use of SERS to detect synthetic cannabinoids in solution.

The SERS method involved the use of a gold nanoparticle solution with the addition of an aggregating agent to detect the synthetic cannabinoids. The aggregating agents examined included chloride, sulfate, and nitrate salts ranging from $0.00835 \mathrm{M}-$ $0.0334 \mathrm{M}$. The initial study with the benchtop Raman spectrometer concluded that $0.0167 \mathrm{M} \mathrm{MgCl}_{2}$ produced the lowest LODs ranging between $18-60 \mathrm{ng} / \mathrm{mL}$ and that each synthetic cannabinoid produced a spectrum that was different from each other.

In addition to the optimization of the SERS method, the study compared the use of a portable and benchtop Raman spectrometer. The SERS spectrums produced from each instrument was compared to Raman spectrum of the powder form of the drug. The results showed that JWH-018 and JWH-073 produced a SERS spectrum that was different between the benchtop and portable instrument as well as the Rama spectrum of the powder form of the drug. However, JWH-081 and JWH-122 produced SERS spectrums that were similar to each other and to the Raman spectrum of the standard. When comparing the LODs, the results concluded that the portable spectrometer can produce LODs similar to that of the benchtop spectrometer. However, a higher $\mathrm{MgCl}_{2}$ concentrated was needed for 3 out of 4 synthetic cannabinoids examined to produce similar LODs. Overall the study concluded, that SERS could be used to detect synthetic cannabinoids at low levels and that a cheaper instrument could be used to produce similar results but require modification to the SERS method.

To determine if a SERS method has been optimized, various synthetic cannabinoids and a wide range of aggregating agents need to be examined. The second 
study expanded to examining nine synthetic cannabinoid parent compounds and assessed the use of monovalent, divalent, and trivalent salts as aggregating agents. In SERS, the aggregating agent is used to destabilize the gold nanoparticles in solution creating 'hot spots' where the Raman signal is enhanced.

Gaining an understanding of the gold nanoparticle and aggregating agent interaction is important in the development of an optimized SERS method. Therefore, a study was conducted analyzing the effect of various salts. Initially, either $0.84 \mathrm{M}$ monovalent salts or $0.05 \mathrm{M}$ monovalent or divalent salts were titrated in $10 \mu \mathrm{L}$ increments to the gold nanoparticle solution to assess the color change (red to purple to grey/clear) and change in the absorbance of the nanoparticle solution via UV-VIS spectroscopy. As the aggregating agent concentration increased, the UV-VIS spectrum produced a decrease in the absorbance and a red shift in the UV-VIS spectrum from $521 \mathrm{~nm}$ to a higher wavelength, which occurred for all the salts examined. Yet, the salt concentration varied at which this transition occurred. A higher salt concentration was needed for monovalent salts compared to divalent and trivalent salts to produce the same effect; however, no significant difference was observed comparing the various anions. This showed that the aggregating agent cation can influence nanoparticle aggregation.

For the second part of the aggregating agent study, the effect on nanoparticle size and zeta potential was examined for each salt at the three concentrations: initial salt concentration added as well as the salt concentrations at which the nanoparticle solution turned purple and grey. For the nanoparticle size study, as the salt was added to the nanoparticle solution, a single peak distribution, seen in stabilized gold nanoparticles, formed two unresolved peak distributions. At the purple color transition, the two peaks 
become more resolved showing the amount of aggregated versus unaggregated nanoparticles. At the grey/clear color transition, a single resolved peak was formed showing that all nanoparticles in solution have aggregated.

For the zeta potential study, upon the addition of the aggregating agent the zeta potential of the nanoparticle solution decreased. As the concentration of the aggregating agent increased, the zeta potential further decreased. This was to be expected. Stabilized nanoparticles have a higher zeta potential value than the recorded average nanoparticle size. Destabilization occurs when the recorded zeta potential is a lower value than the average nanoparticle size. The results both studies were seen for all the aggregating agents examined. While the previous studies only focused on the effects of monovalent cationic salts on aggregation, this study focused on the effects of monovalent, divalent, and trivalent cationic salts. The results obtained for all the salts examined results agree with the Schulze-Hardy rule. The higher the valence of the aggregating agent cation, $\mathrm{Al}^{3+,}$ the least amount of salt needed to produce the CCC for gold nanoparticles.

After gaining an understanding of the aggregating agent and gold nanoparticle interaction, nine different synthetic cannabinoids were examined using the various salts at the determined CCC. Out of all the salts examined, $0.0015 \mathrm{MgCl}_{2}$ was the optimal aggregating agent with limits of detection (LOD) ranging from 20 to $130 \mathrm{ng} / \mathrm{mL}$. These LODs agree with initial study. Therefore, showing that a lower $\mathrm{MgCl}_{2}$ concentration can be used to produce similar results.

The final portions of this study fused on the practical application of the developed SERS method to detect synthetic cannabinoid metabolites in spiked urine samples. The parent compounds were not analyzed in this study because they are not likely to be found 
in urine samples. Initially, the optimized SERS method developed in chapter 4 was used to detect two JWH-018 metabolite standards in a $10 \% \mathrm{MeOH}$ solution producing an LOD as low as $37 \mathrm{ng} / \mathrm{mL}$. This showed that both parent compounds and metabolites of synthetic cannabinoids could be detected using this SERS method. Then, urine samples spiked with the JWH-018 metabolites at various concentrations were examined using SLE or $\mu$-SPE as the extraction method. Despite varying the urine sample pretreatments and the addition of acidic $\mathrm{MeOH}$ after the extraction, no variation of the SLE method produced a SERS spectrum that was different to that of the negative control. However, the SERS spectrum of the JWH-018 metabolites could be produced using $\mu$-SPE with a LOD as low as $650 \mathrm{ng} / \mathrm{mL}$.

Further study needs to be conducted to optimize the $\mu$-SPE method examined in this study to obtain a LOD similar to the results achieved in chapter 4. Adjusting the $\mathrm{pH}$ of the urine sample prior to being washed with the ZipTip pipette tip could help with the extraction. An adjustment of the ratio of nanoparticles to aggregating agent to drug need to be examined. The optimized method in chapter 4 has a 1:100 dilution of the drug in the analyzed nanoparticle solution. Therefore, increasing concentration of the analyte of interest, could increase the recovery of the sample after the extraction and lower the LOD.

Another factor to consider is how to gain a better understanding of the success of the method. It is done by calculating the LOD. In the chapters examined in my study, the LOD was calculated using a single peak in the SERS spectrum using a specific dynamic range in which a linear fit was optimal for the parent compounds and a logarithmic fit was optimal for the JWH-018 metabolites. Further study can be conducted on using a 
ratio of the Raman signal intensity of multiple peaks in the SERS spectrum. A recent study showed that using a ratio of the Raman signal intensity at two peaks in the SERS spectrum provided a better curve fit to the wide concentration range examined. Instead of a linear fit, a Langmuir adsorption curve was seen. ${ }^{87}$

Once an optimized extraction method has been developed, additional synthetic cannabinoid metabolites need to be examined as well as metabolites of cathinones and opioids. If the above compounds can be detected using this optimized SERS method, then further studies need to be conducted on additional biological matrixes such as oral fluid and blood. Our research group was able to detect JWH-018 in spiked oral fluid samples at a LOD of $0.8 \mathrm{ng} / \mathrm{mL} .{ }^{87}$ Additional compounds need to be examined in oral fluid samples. If this developed SERS method with gold nanoparticles cannot be used to detect all the above-mentioned compounds, then the next step will be to examine other nanoparticles of various shapes and metal composition.

Raman spectroscopy provides spectrum that can be used to interpret unknown compounds that could give a false negative if tested via an immunoassay. Overall the SERS method has demonstrated that synthetic cannabinoids can be detected at concentrations as low as $20 \mathrm{ng} / \mathrm{mL}$. 


\section{LIST OF REFERENCES}

1. Castaneto, M. S.; Wohlfarth, A.; Desrosiers, N. A.; Hartman, R. L.; Gorelick, D. A.; Huestis, M. A., Synthetic cannabinoids pharmacokinetics and detection methods in biological matrices. Drug Metab Rev. 2015, 47 (2):124-74.

2. Davidson C.; Opacka-Juffry J.; Arevalo-Martin A.; Garcia-Ovejero D.; Molina-Holgado E.; Molina-Holgado F. Spicing up pharmacology: a review of synthetic cannabinoids from structure to adverse events. Adv Pharmacol. 2017, 80, 135-168.

3. Seely, K. A.; Lapoint, J.; Moran, J. H.; Fattore, L. Spice drugs are more than harmless herbal blends: a review of the pharmacology and toxicology of synthetic cannabinoids. Prog Neuro-Psychoph. 2012, 39, 234-24.

4. Shevyrin, V.; Melkozerov, V.; Endres, G. W.; Shafran, Y.; Morzherin, Y. On a new cannabinoid classification system: a sight on the illegal market of novel psychoactive substances. Cannabis and Cannabinoid Research. 2016, 1, 18694.

5. Gurdal, F.; Asirdizer, M.; Aker, R. G.; Korkut, S.; Gocer, Y.;

Kucukibrahimoglu, E. E.; Ince, C. H. Review of detection frequency and type of synthetic cannabinoids in herbal compounds analyzed by Istanbul Narcotic Department of the Council of Forensic Medicine, Turkey. J Forensic Leg Med. 2013, 20, 667-72.

6. Brents, L. K.; Reichard, E. E.; Zimmerman, S. M.; Moran, J. H.; Fantegrossi, W. E.; Prather, P. L. Phase I hydroxylated metabolites of the K2 synthetic cannabinoid JWH-018 retain in vitro and in vivo cannabinoid 1 receptor affinity and activity. PloS one. 2011, 6, e21917.

7. Kacinko, S. L.; Xu, A.; Homan, J. W.; McMullin, M. M.; Warrington, D. M.; Logan, B. K. Development and validation of a liquid chromatography-tandem mass spectrometry method for the identification and quantification of JWH018, JWH-073, JWH-019, and JWH-250 in human whole blood. J Anal Toxicol. 2011, 35, 386-93.

8. de Jager, A. D.; Warner, J. V.; Henman, M.; Ferguson, W.; Hall A. LCMS/MS method for the quantitation of metabolites of eight commonly-used synthetic cannabinoids in human urine-an Australian perspective. J Chromato B. 2012, 897, 22-31.

9. Kneisel, S.; Auwärter, V.; Kempf, J. Analysis of 30 synthetic cannabinoids in oral fluid using liquid chromatography-electrospray ionization tandem mass spectrometry. Drug Test Anal. 2013, 5, 657-69. 
10. Hutter, M.; Moosmann, B.; Kneisel, S.; Auwärter, V. Characteristics of the designer drug and synthetic cannabinoid receptor agonist AM-2201 regarding its chemistry and metabolism. J Mass Spectrom. 2013, 48, 885-94.

11. Hage D. S. Immunoassays. Anal Chem. 1999, 71, 294-304.

12. Levine, B., Principles of forensic toxicology. $2^{\text {nd }}$ Ed.; AACC Press: Washinton, DC, 2003.

13. Rodrigues, W.C.; Catbagan, P.; Rana, S.; Wang, G.; Moore, C. Detection of synthetic cannabinoids in oral fluid using ELISA and LC-MS-MS. J Anal Toxicol. 2013, 37, 526-33.

14. Arntson, A.; Ofsa, B.; Lancaster, D.; Simon, J.R.; McMullin, M.; Logan, B. Validation of a novel immunoassay for the detection of synthetic cannabinoids and metabolites in urine specimens. J Anal Toxicol. 2013, 37, 284-90.

15. Barnes, A.J.; Young, S.; Spinelli, E.; Martin, T. M.; Klette, K. L.; Huestis, M. A. Evaluation of a homogenous enzyme immunoassay for the detection of synthetic cannabinoids in urine. Forensic Sci Int. 2014, 241, 27-34.

16. Wang, G.; Huynh, K.; Barhate, R.; Rodrigues, W.; Moore, C.; Coulter, C.; Vincent, M.; Soares, J. Validation of a new homogeneous immunoassay for the detection of carisoprodol in urine. J Anal Toxicol. 2011, 35, 108-12.

17. Swortwood, M.J. Comprehensive forensic toxicological analysis of designer drugs. Dissertation. Florida International University. 2013.

18. Kronstrand, R.; Brinkhagen, L.; Birath-Karlsson, C.; Roman, M.; Josefsson, M. LC-QTOF-MS as a superior strategy to immunoassay for the comprehensive analysis of synthetic cannabinoids in urine. Anal Bioanal Chem. 2014, 406, 3599-3609.

19. Robards, K.; Robards, K.; Haddad, P. R.; Haddad, P. R.; Jackson, P. E.; Jackson PE, Haddad PA. Principles and practice of modern chromatographic methods. Elsevier Academic Press: San Diego, 1994.

20. Kavanagh, P.; Grigoryev, A.; Melnik, A.; Simonov, A. The identification of the urinary metabolites of 3-(4-methoxybenzoyl)-1-pentylindole (RCS-4), a novel cannabimimetic, by gas chromatography-mass spectrometry. J Anal Toxicol. 2012, 36, 303-311. 
21. Grigoryev, A.; Kavanagh, P.; Melnik, A.; Savchuk, S.; Simonov, A. Gas and liquid chromatography-mass spectrometry detection of the urinary metabolites of UR-144 and its major pyrolysis product. J Anal Toxicol. 2013, 37, 265-76.

22. Heltsley, R.; Shelby, M. K.; Crouch, D. J.; Black, D. L.; Robert, T. A.; Marshall, L.; Bender, C. L.; DePriest, A. Z.; Colello, M. A. Prevalence of synthetic cannabinoids in US athletes: initial findings. J Anal Toxicol. 2012, 36, 588-593.

23. Scheidweiler, K. B.; Jarvis, M. J.; Huestis, M. A. Nontargeted SWATH acquisition for identifying 47 synthetic cannabinoid metabolites in human urine by liquid chromatography-high-resolution tandem mass spectrometry. Anal Bioanal Chem. 2015, 407, 883-897.

24. Ferraro, J. R.; Nakamoto, K. Introductory Raman Spectroscopy, 1st ed.; Academic Press, INC.: San Diego, 1994.

25. Ru, E. L.; Etchegoin, P. Principles of Surfaced Enhanced Raman Spectroscopy and related plasmonic effects, 1 st ed.; Elsevier: Amsterdam, Netherlands, 2009.

26. Skoog, D. A.; Holler, F. J.; Nieman, T. A., Principles of instrumental analysis, $6^{\text {th }}$ ed.; Hartcourt Brace \& Company: Philadelphia, 1998.

27. Weyermann, C.; Mimoune, Y.; An glada, F.; Massonnet, G.; Esseiva, P.; Buzzini, P. Applications of a transportable Raman spectrometer for the in situ detection of controlled substances at border controls. Forensic Sci Int. 2011, 209, 21-28.

28. Fleischmann, M.; Hendra, P. J.; McQuillan, A. J. Raman spectra of pyridine adsorbed at a silver electrode, Chem Phys Lett 1974, 26, 163-166.

29. Thanh, N. T.; Maclean, N.; Mahiddine, S. Mechanisms of nucleation and growth of nanoparticles in solution. Chem Rev. 2014, 114, 7610-7630.

30. Chow, M. K.; Zukoski, C. F. Gold sol formation mechanisms: role of colloidal stability. J Colloid Interf Sci. 1994, 165, 97-109.

31. Park, J. W.; Shumaker-Parry, J. S. Structural study of citrate layers on gold nanoparticles: role of intermolecular interactions in stabilizing nanoparticles. $J$ Am Chem Soc. 2014, 136, 1907-1921.

32. Etchegoin, P. G.; Le RU, E. C. Basic Electromagnetic Theory of SERS. In Surface Enhanced Raman Spectroscopy: Analytical, Biophysical, and Life 
Science Applications. Schlücker, S.. Ed.; Wiley-VCH: Weinheim, Germany, 2011; pp 1-37.

33. Kim, T.; Lee, C. H.; Joo, S. W.; Lee, K. Kinetics of gold nanoparticle aggregation: experiments and modeling. J Colloid Interf Sci. 2008, 318, 23843.

34. Burns, C.; Spendel, W. U.; Puckett, S.; Pacey, G. E. Solution ionic strength effect on gold nanoparticle solution color transition. Talanta. 2006, 69, 873876.

35. Oncsik, T.; Trefalt, G.; Borkovec, M.; Szilagyi, I. Specific ion effects on particle aggregation induced by monovalent salts within the Hofmeister series. Langmuir. 2015, 31, 3799-807.

36. Pavlovic, M.; Huber, R.; Adok-Sipiczki, M.; Nardin, C.; Szilagyi, I. Ion specific effects on the stability of layered double hydroxide colloids. Soft Matter. 2016, 12, 4024-33.

37. Bell, S. E.; Stewart, S. P.; Ho, Y. C.; Craythorne, B. W.; Speers, S. J. Comparison of the discriminating power of Raman and surface-enhanced Raman spectroscopy with established techniques for the examination of liquid and gel inks. J Raman Spectrosc. 2013, 44, 509-517.

38. Rodger, C.; Dent, G.; Watkinson, J.; Smith, W. E. Surface-enhanced resonance Raman scattering and near-infrared Fourier transform Raman scattering as in situ probes of ink jet dyes printed on paper. Appl Spectrosc. 2000, 54, 1567-1576.

39. Idone, A.; Gulmini, M.; Henry, A. I.; Casadio, F.; Chang, L.; Appolonia, L.; Van Duyne, R. P.; Shah, N. C. Silver colloidal pastes for dye analysis of reference and historical textile fibers using direct, extractionless, nonhydrolysis surface-enhanced Raman spectroscopy. Analyst. 2013, 138, 58955903.

40. Chen, T. F.; Lu, S. H.; Wang, A. J.; Zheng, D.; Wu, Z. L.; Wang, Y. S. Detection of explosives by surface enhanced Raman scattering using substrate with a monolayer of ordered Au nanoparticles. Appl Surf Sci. 2014, 317, 940945 .

41. Hoppmann, E. P.; Yu, W. W.; White, I. M. Detection of deoxyribonucleic acid (DNA) targets using polymerase chain reaction (PCR) and paper surfaceenhanced Raman spectroscopy (SERS) chromatography. Appl Spectrosc. 2014, 68, 909-915. 
42. Ngo, Y. H.; Li, D.; Simon, G. P.; Garnier, G. Gold nanoparticle-paper as a three-dimensional surface enhanced raman scattering substrate. Langmuir. 2012, 28, 8782-8790.

43. Cinta, S.; Iliescu, T.; Astilean, S.; David, L.; Cozar, O.; Kiefer, W. 1, 4Benzodiazepine drugs adsorption on the Ag colloidal surface. J Mol Struct. 1999, 482, 685-688.

44. Doctor, E. L.; McCord, B. Comparison of aggregating agents for the surfaceenhanced Raman analysis of benzodiazepines. Analyst. 2013, 138, 5926-5932.

45. Doctor, E. L.; McCord, B. The application of supported liquid extraction in the analysis of benzodiazepines using surface enhanced Raman spectroscopy. Talanta. 2015, 144, 938-943.

46. Stewart, S.P.; Bell, S.E.; Fletcher, N. C.; Bouazzaoui, S.; Ho, Y. C.; Speers, S. J.; Peters, K. L. Raman spectroscopy for forensic examination of $\beta$ ketophenethylamine "legal highs": Reference and seized samples of cathinone derivatives. Anal Chim Acta. 2012, 711, 1-6.

47. Mabbott, S.; Correa, E.; Cowcher, D. P.; Allwood, J. W.; Goodacre, R. Optimization of parameters for the quantitative surface-enhanced raman scattering detection of mephedrone using a fractional factorial design and a portable Raman spectrometer. Anal Chem. 2013, 85, 923-931.

48. Farquharson, S.; Shende, C.; Sengupta, A.; Huang, H.; Inscore, F. Rapid detection and identification of overdose drugs in saliva by surface-enhanced Raman scattering using fused gold colloids. Pharmaceutics. 2011, 3, 425-439.

49. Islam, S. K.; Cheng, Y. P.; Birke, R. L.; Green, O.; Kubic, T.; Lombardi, J. R. Rapid and sensitive detection of synthetic cannabinoids AMB-FUBINACA and $\alpha$-PVP using surface enhanced Raman scattering (SERS). Chem Phys. 2018, 506, 31-35.

50. Alkaseem, M.; Baron, M. SERS and DFT study of 5F-PB-22. J Raman Spectrosc. 2018, 49, 1594-1606.

51. Office of National Drug Control Policy, Synthetic Drugs (a.k.a. K2, Spice, Bath Salts, etc.) 〈http://www.whitehouse.gov/ondcp/ondcp-factsheets/synthetic-drugs-k2spice-bath-salts $\rangle$, (accessed 7.10.16)

52. D.M. Bush, D.A. Woodwell, Substance Abuse and Mental Health Services Administration, The CBHSQ report: Update: drug-related emergency department visits involving synthetic cannabinoids 
〈http://www.samhsa.gov/data/sites/ default/files/SR-1378/SR-1378.pdf〉

(accessed 21.9.16)

53. European Monitoring Centre for Drugs and Drug Addiction, European drug report 2016: trends and developments, Publications Office of the European Union, Luxembourg

〈http://www.emcdda.europa.eu/system/files/publications/2637/

TDAT16001ENN.pdf $\rangle, 2016$ (accessed 20.5.16)

54. The challenge of synthetic drugs in East and South-East Asia and Oceania: trends and patterns of amphetamine-type stimulants and new psychoactive substances, A report from the global SMART programme, United Nations Office on Drugs and Crime 〈http://www.unodc.org/documents/southeastasiaandpacific/Publications/ 2015/drugs/ATS_2015_Report_web.pdf), May 2015 (accessed 20.5.16)

55. H. Williams, Synthetic marijuana, other designer drugs enter United States legally, CBS News, cbsnews.com 〈http://www.cbsnews.com/news/syntheticmarijuana-other-designer-drugsenter-united-states-legally/>, 4.12.13 (accessed 19.5.16)

56. Choi, H.; Heo, S.; Choe, S.; Yang, W.; Park, Y.; Kim, E.; Chung, H.; Lee, J. Simultaneous analysis of synthetic cannabinoids in the materials seized during drug trafficking using GC-MS. Anal Bioanal Chem 2013, 405, 3937-3944.

57. Namera, A.; Kawamura, M.; Nakamoto, A.; Saito, T.; Nagao, M. Comprehensive review of the detection methods for synthetic cannabinoids and cathinones. Forensic Toxicol. 2015, 33, 175-194.

58. Nieddu, M.; Trignano, C.; Burrai, L.; Pirisi, M. A.; Boatto, G. Crossreactivities of 41 new amphetamine designer drugs to EMIT® immunoassays. Forensic Toxicol. 2013, 31, 133-137.

59. Spinelli, E.; Barnes, A. J.; Young, S.; Castaneto, M. S.; Martin, T. M.; Klette, K. L.; Huestis, M. A. Performance characteristics of an ELISA screening assay for urinary synthetic cannabinoids. Drug Test Anal. 2015, 7, 467-474.

60. Shanks, K. G.; Behonick, G. S.; Dahn, T.; Terrell, A. Identification of novel thirdgeneration synthetic cannabinoids in products by ultra-performance liquid chromatography and time-of-flight mass spectrometry. J Anal Toxicol. 2013, 37, 517-525.

61. Hermanns-Clausen, M.; Kneisel, S.; Szabo, B.; Auwärter, V. Acute toxicity due to the confirmed consumption of synthetic cannabinoids: clinical and laboratory findings. Addiction. 2013, 108, 534-544. 
62. Lee, P. C.; Meisel, D. Adsorption and surface-enhanced Raman on silver and gold sols. J Phys Chem. 1982, 86, 3391-3395.

63. Izake, E. L. Forensic and homeland security applications of modern portable Raman spectroscopy. Forensic Sci Int. 2010, 202, 1-8.

64. Muehlethaler, C.; Leona, M.; Lombardi, J. R. Towards a validation of surfaceenhanced Raman scattering (SERS) for use in forensic science: repeatability and reproducibility experiments. Forensic Sci Int. 2016, 268, 113.

65. Fedchak, S. Presumptive Field Testing Using Portable Raman Spectroscopy. Las Vegas: US Department of Justice. 2014.

66. Sägmüller, B.; Schwarze, B.; Brehma, G.; Schneider, S. Application of SERS spectroscopy to the identificationof (3,4-methylenedioxy) amphetamine in forensic samples utilizing matrix stabilized silver halides. Analyst. 2001, 126, 2066-2071.

67. Berg, R. W.; Nørbygaard, T.; White, P. C.; Abdali, S. Ab initio calculations and Raman and SERS spectral analyses of amphetamine species. Appl Spectrosc Rev. 2011, 46, 107-131.

68. Taplin, F.; O'Donnell, D.; Kubic, T.; Leona, M.; Lombardi, J. Application of Raman spectroscopy, surface-enhanced raman scattering (SERS), and density functional theory for the identification of phenethylamines. Appl Spectrosc 2013, 67, 1150-1159.

69. Sägmüller, B.; Schwarze, B.; Brehm, G.; Trachta, G.; Schneider, S. Identification of illicit drugs by a combination of liquid chromatography and surface-enhanced Raman scattering spectroscopy. J Mol Struct. 2003, 661662, 279-290.

70. Trachta, G.; Schwarze, B.; Sägmüller, B.; Brehm, G.; Schneider, S. Combination of high-performance liquid chromatography and SERS detection applied to the analysis of drugs in human blood and urine. J Mol Struct. 2004, $693,175-185$.

71. Gambinossi, F.; Mylon, S. E.; Ferri, J. K. Aggregation kinetics and colloidal stability of functionalized nanoparticles. Adv Colloid Interface Sci. 2015, 222, 332-49.

72. Mikhlin, Y.; Karacharov, A.; Likhatski, M.; Podlipskaya, T; Zubavichus, Y.; Veligzhanin, A.; Zaikovski, V. Submicrometer intermediates in the citrate 
synthesis of gold nanoparticles: New insights into the nucleation and crystal growth mechanisms. J. Colloid Interface Sci. 2011, 362, 330-336.

73. Wang, F.; Richards, V. N.; Shields, S. P.; Buhro, W. E. Kinetics and mechanisms of aggregative nanocrystal growth. Matter. 2013, 26, 5-21.

74. Bell, S.E.; McCourt, M.R. SERS enhancement by aggregated Au colloids: effect of particle size. Phys Chem Chem Phys. 2009, 11, 7455-7462.

75. Virkler, K.; Lednev, I. K. Blood species identification for forensic purposes using Raman spectroscopy combined with advanced statistical analysis. Anal Chem. 2009, 81, 7773-7777.

76. Muehlethaler, C.; Cheng, Y. P.; Islam, S. K.; Lombardi, J. R. Contribution of Raman and Surface Enhanced Raman Spectroscopy (SERS) to the analysis of vehicle headlights: Dye (s) characterization. Forensic Sci Int. 2018, 287, 98 107.

77. Zaffino, C.; Ngo, H. T.; Register, J.; Bruni, S.; Vo-Dinh, T. "Dry-state" surface-enhanced Raman scattering (SERS): toward non-destructive analysis of dyes on textile fibers. Appl Phys A. 2016, 122, 707.

78. Hakonen, A.; Andersson, P. O.; Schmidt, M. S.; Rindzevicius, T.; Käll M. Explosive and chemical threat detection by surface-enhanced Raman scattering: A review. Anal Chim Acta. 2015, 893, 1-3.

79. Dong, R.; Weng, S.; Yang, L.; Liu, J. Detection and direct readout of drugs in human urine using dynamic surface-enhanced Raman spectroscopy and support vector machines. Anal. Chem. 2015, 87, 2937-2944.

80. Alharbi, O.; Xu, Y.; Goodacre, R. Detection and quantification of the opioid tramadol in urine using surface enhanced Raman scattering. Analyst, 2015, 140, 5965-5970.

81. Ma, Y.; Liu, H.; Mao, M.; Meng, J.; Yang, L.; Liu, J. Surface-enhanced Raman spectroscopy on liquid interfacial nanoparticle arrays for multiplex detecting drugs in urine. Anal Chem. 2016, 88, 8145-8151.

82. Meng, J.; Tang, X.; Zhou, B.; Xie, Q.; Yang, L. Designing of ordered twodimensional gold nanoparticles film for cocaine detection in human urine using surface-enhanced Raman spectroscopy. Talanta. 2017, 164, 693-699.

83. Han, Z.; Liu, H.; Meng, J.; Yang, L.; Liu, J.; Liu, J. Portable kit for identification and detection of drugs in human urine using surface-enhanced Raman spectroscopy. Anal Chem. 2015, 87, 9500-9506. 
84. Andreou, C.; Hoonejani, M. R.; Barmi, M. R.; Moskovits, M.; Meinhart, C. D. Rapid detection of drugs of abuse in saliva using surface enhanced Raman spectroscopy and microfluidics. ACS Nano. 2013, 7, 7157-7164.

85. Dana, K.; Shende, C.; Huang, H.; Farquharson, S. Rapid analysis of cocaine in saliva by surface-enhanced Raman spectroscopy. J Anal Bioanal Technol. 2015, 6, 1-5.

86. Mostowtt, T.; McCord, B. Surface enhanced Raman spectroscopy (SERS) as a method for the toxicological analysis of synthetic cannabinoids. Talanta, 2017, 164, 396-402.

87. Deriu, C.; Conticello, I.; Mebel, A. M.; McCord, B. Micro Solid Phase Extraction Surface-Enhanced Raman Spectroscopy ( $\mu$-SPE/SERS) Screening Test for the Detection of the Synthetic Cannabinoid JWH-018 in Oral Fluid. Anal Chem. 2019, 91, 4780-4789.

88. Sengel, T. Y.; Celik, E. G.: Aydogan, C.; Gumus, Z. P.; Ilktac, R.; Aydindogan, E.; Ciftci, M.; Aldemir, E.; Coskunol, H.; Timur, S.; Yagci, Y. A Functional Platform for the Detection of JWH-073 as a Model for Synthetic Cannabinoids. ChemElectroChem. 2018, 5, 1253- 12538.

89. Spinelli, E.; Barnes, A. J.; Young, S.; Castaneto, M. S.; Martin, T. M.; Klette, K. L.; Huestis, M. A. Performance characteristics of an ELISA screening assay for urinary synthetic cannabinoids. Drug Test Anal. 2015, 7, 467-474.

90. Coglitore, D.; Edwardson, S. P.; Macko, P.; Patterson, E. A.; Whelan, M. Transition from fractional to classical Stokes-Einstein behaviour in simple fluids. $R$ Soc Open Sci. 2017, 4, 170507.

91. Kaszuba, M.; Corbett, J.; Watson, F. M.; Jones, A. High-concentration zeta potential measurements using light-scattering techniques. Philos Trans $R$ Soc. A. 2010, 368, 4439-4451.

92. Badawy, A. M.; Luxton, T. P.; Silva, R. G.; Scheckel, K. G.; Suidan, M. T.; Tolaymat, T. M. Impact of environmental conditions ( $\mathrm{pH}$, ionic strength, and electrolyte type) on the surface charge and aggregation of silver nanoparticles suspensions. Environ Sci Technol. 2010, 44, 1260-1266.

93. Li, X.; Lenhart, J. J.; Walker, H. W. Aggregation kinetics and dissolution of coated silver nanoparticles. Langmuir. 2012, 28, 1095-104. 
94. López-León, T.; Jódar-Reyes, A. B.; Bastos-González, D.; Ortega-Vinuesa, J. L. Hofmeister effects in the stability and electrophoretic mobility of polystyrene latex particles. $J$ Phys Chem B, 2003, 107, 5696- 5708.

95. Merk, V.; Rehbock, C.; Becker, F.; Hagemann, U.; Nienhaus, H.; Barcikowski, S. In situ non-DLVO stabilization of surfactant-free, plasmonic gold nanoparticles: Effect of Hofmeister's anions. Langmuir. 2014, 30, 4213 4422 .

96. Tian, R.; Yang, G.; Li, H.; Gao, X.; Liu, X.; Zhu, H.; Tang, Y. Activation energies of colloidal particle aggregation: towards a quantitative characterization of specific ion effects. Phys Chem Chem Phys. 2014, 16, 8828-8836.

97. Rouster, P.; Pavlovic, M.; Szilagyi, I. Destabilization of titania nanosheet suspensions by inorganic salts: Hofmeister series and Schulze-Hardy rule. $J$. Phys Chem B. 2017, 121, 6749-6758.

98. Trefalt, G.; Szilagyi, I.; Téllez, G.; Borkovec, M. Colloidal stability in asymmetric electrolytes: modifications of the Schulze-Hardy rule. Langmuir. 2017, 33, 1695-1704.

99. Supported Liquid Extraction (SLE) Guidde and FAQ's. Thermo Scientific. https://assets.thermofisher.com/TFS-Assets/CMD/manuals/TG-21068-SPSupported-Liquid-Extraction-SLE-User-FAQ-TG21068-EN.pdf

100. Mostowtt, T.; Munoz, J; McCord, B. An evaluation of monovalent, divalent, and trivalent cations as aggregating agents for surface enhanced Raman spectroscopy (SERS) analysis of synthetic cannabinoids. Analyst. 2019, 144, 6404-14.

101. Sun, Q.; Zheng, H. Raman OH stretching vibration of ice Ih. Prog Nat Sci. 2009, 19, 1651-1654.

102. Munshi, T.; Brewster, V. L.; Edwards, H. G.; Hargreaves, M. D.; Jilani, S. K.; Scowen, I. J. Monitoring of the interconversion of gamma-butyrolactone (GBL) to gamma hydroxybutyric acid (GHB) by Raman spectroscopy. Drug Test Anal. 2013, 5, 678-82. 
VITA

\title{
THADDEUS H. MOSTOWTT
}

\author{
Born, San Diego, California
}

2006-2010

B.S., Chemistry

Mount St. Mary's University

Emmitsburg, Maryland

2010-20012

M.F.S., Forensic Chemistry

George Washington University

Washington, District of Columbia

2016

Research Assistant

Florida International University

Miami Florida

2013-2015

Teaching Assistant

Florida International University

Miami Florida

2016-2019

Doctoral Candidate

Florida International University

Miami Florida

\section{PUBLICATIONS AND PRESENTATIONS}

Thaddeus H. Mostowtt, Robert Ramotowski, and John Morgan Jr. "A Comparison of Thermal Fingerprint Development to Current Recommended Chemical Development Techniques on Porous Surfaces." Journal of Forensic Identification. 66, 4 (2016), 326348.

Thaddeus H. Mostowtt and Bruce McCord "Surface enhanced Raman spectroscopy (SERS) as a method for the toxicological analysis of synthetic cannabinoids," Talanta, 164 (2017), 396-402.

Thaddeus H. Mostowtt, Jonathan Munoz and Bruce McCord "An evaluation of monovalent, divalent, and trivalent cations as aggregating agents for surface enhanced Raman spectroscopy (SERS) analysis of synthetic cannabinoids," Analyst, 144, (2019), 6404-6414.

Thaddeus H. Mostowtt, Robert Ramotowski, and John Morgan Jr, "A Comparison of Thermal Fingerprint Development to Known Chemical Techniques on Porous Surfaces." 
2014, Miami, Fl. Poster presentation. $3^{\text {rd }}$ Annual Forensic Science Symposium, International Forensic Research Institute.

Thaddeus H. Mostowtt and Bruce McCord. "Surface-Enhanced Raman Analysis of Synthetic Cannabinoids Using Gold Nanoparticles and Various Aggregating Agents." 2015, Orlando, Fl. Poster presentation. American Academy of Forensic Sciences. Miami, Fl. $4^{\text {th }}$ Annual Forensic Science Symposium, International Forensic Research Institute.

Thaddeus H. Mostowtt and Bruce McCord. "The Application of Gold Nanoparticles for the Trace Detection of PINACAs in Urine by Surface Enhanced Raman Spectroscopy (SERS).” 2016, Las Vegas, NV, Poster presentation. American Academy of Forensic Sciences. Miami, Fl. $5^{\text {th }}$ Annual Forensic Science Symposium, International Forensic Research Institute.

Thaddeus H. Mostowtt and Bruce McCord. "The Evaluation of Mono-, Di-, and Trivalent Cations for the Optimized Surface Enhanced Raman Spectroscopy (SERS) Enhancement to Detect Synthetic Cannabinoids in Biological Samples." 2017, New Orleans, LA. Poster presentation. American Academy of Forensic Sciences. 